\title{
A STANDOFF APPROACH TO MONITORING INFANT APNEA
}

\author{
A Thesis \\ Presented to \\ the Faculty of California Polytechnic State University, \\ San Luis Obispo
}

\author{
In Partial Fulfillment \\ of the Requirements for the Degree \\ Master of Science in Engineering \\ With a Specialization in Biomedical Engineering
}

by

Lauren Jean West

June 2010 
(C)2010

Lauren Jean West

ALL RIGHTS RESERVED 


\section{COMMITTEE MEMBERSHIP}

TITLE:

AUTHOR:

DATE SUBMITTED:

COMMITTEE CHAIR:

COMMITTEE MEMBER:

COMMITTEE MEMBER:
A Standoff Approach to Monitoring Infant Apnea

Lauren Jean West

June 2010

Dr. Lily Laiho

Dr. David Clague

Dr. Robert Crockett 


\begin{abstract}
A STANDOFF APPROACH TO MONITORING INFANT APNEA

By

Lauren Jean West
\end{abstract}

The focus of this thesis was to capture and measure carbon dioxide concentrations upon exhalation to determine if an apnea event is occurring. Research in the fields of respiratory physiology and apnea built the foundation for the design of the standoff infant apnea monitor. The monitor is designed to track infant respiration using carbon dioxide and sound signatures of breath without touching the infant. Each detection system, audio and carbon dioxide, were designed separately and brought together for a final proof-of-concept device. The software was developed using LabView and run on a Netbook. Testing was conducted on healthy adults to fine tune the carbon dioxide sensor and measure its response during simulated apnea events. Overnight testing showed that the combined system detected fewer false alarms than either system alone. Infant testing was conducted to determine if the proof-of-concept standoff monitor could detect infant breath at specified distance. The results showed that both detection systems can detect infant breath consistently at distances less than one foot from the infant and poorly at distances exceeding one foot. Finally, conclusions were drawn and interpreted to aid in the design of future generations of the standoff infant apnea monitor. Other research avenues where this technology may be useful were also discussed. 


\section{ACKNOWLEDGEMENTS}

There are many people that aided in the success of this thesis. First I would like to thank Dan White and Scott Garrett for their efforts on this project. Dan is very skilled in the area of programming and processing. Scott has a passion for design and is very creative. Together we designed and built a working prototype. Without them this project would not have come together and I learned a lot from both of them.

I would also like to thank my advisor, Dr. Lily Laiho. She is the one who approached me about this project back in 2008. She coordinated the other students involved and allowed us to use her office for our biweekly conference calls with Raytheon. She has also had a part in advising during the writing of this thesis and guidance throughout the project.

I would also like to thank the engineers at Raytheon who brought this project and the finances for it to the table: John Jacobs, Aaron Stein, and Robert McPhie. They helped guide us along the way with biweekly conference calls and helped build connections to Dr. Steven Barkley at Cottage Hospital in Goleta. Dr. Barkley, who is the head of the Neonatal Intensive Care Unit (NICU) at Cottage Hospital, was a major asset during the research and again during the testing phase. He gave up his time to discuss his experience with infant apnea and audio technologies.

Last but not least, I want to thank my family for supporting me throughout the five years of my undergraduate degree in Biomedical Engineering and another year of my Master's of Science in Engineering with a specialization in Biomedical Engineering. I know it was a financial sacrifice for my parents to put me through college and Graduate School. Both my parents and my brother have listened to many hours of me explaining the projects and giving input. Thanks, Andrew, for your support as a brother and one of my best friends. 


\section{Table of Contents}

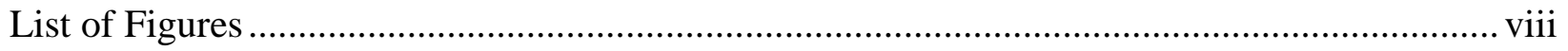

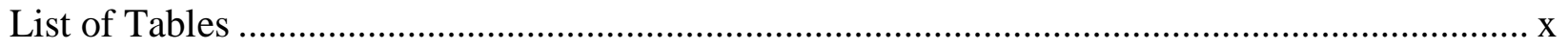

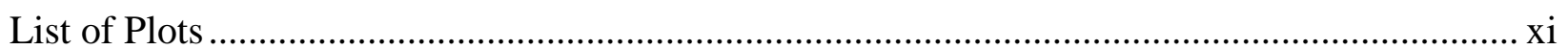

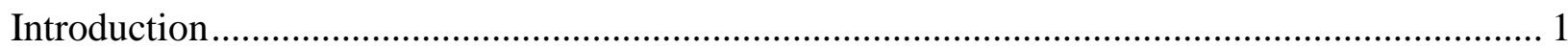

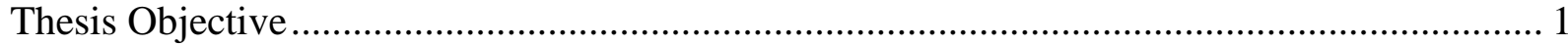

Thesis Purpose

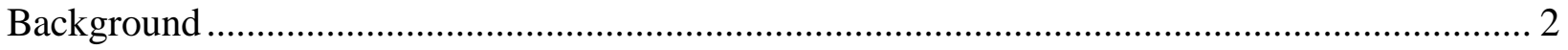

Why Monitor Infant Breathing? .................................................................................... 2

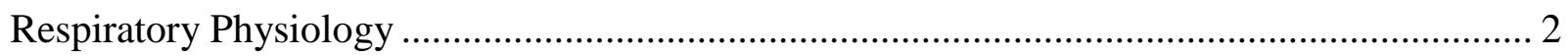

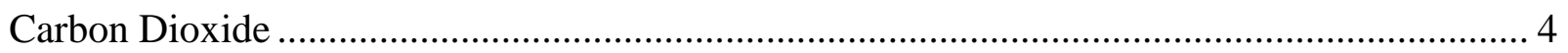

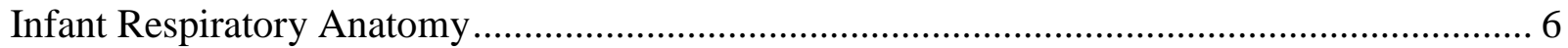

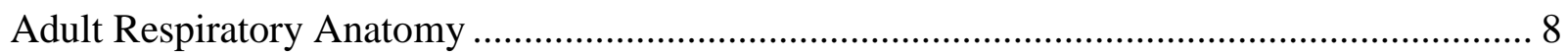

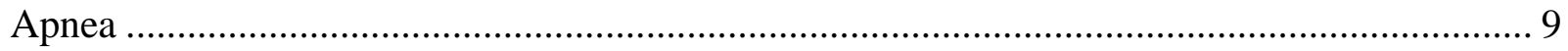

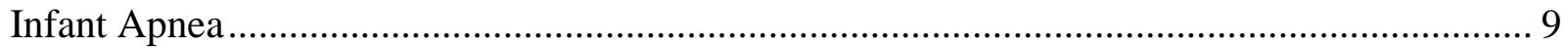

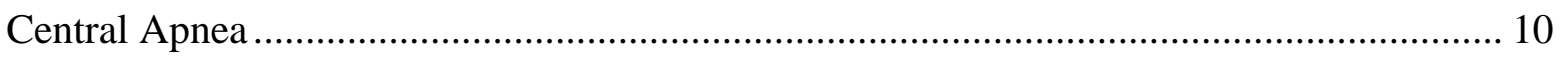

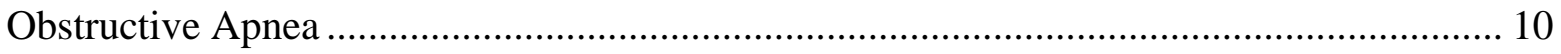

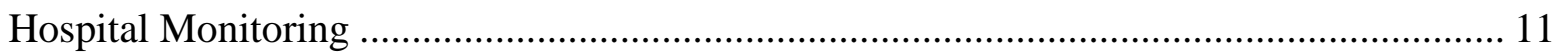

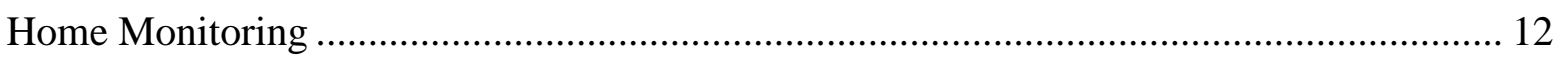

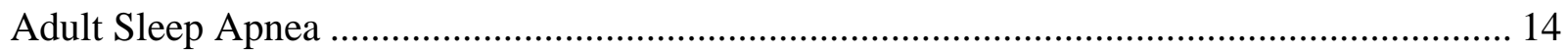

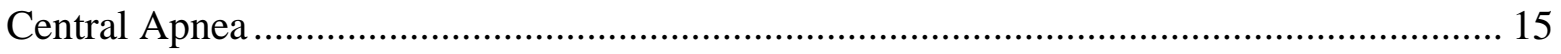

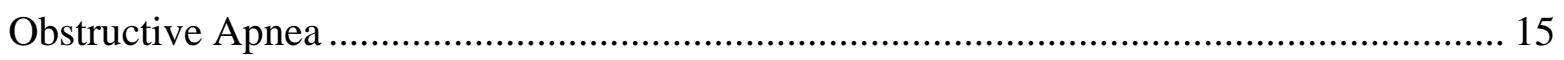

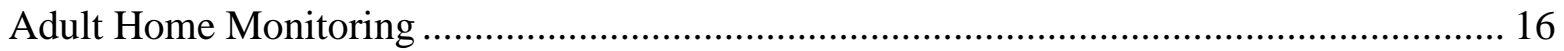

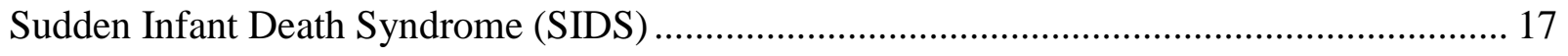

Standoff Monitoring Technologies .................................................................................. 18

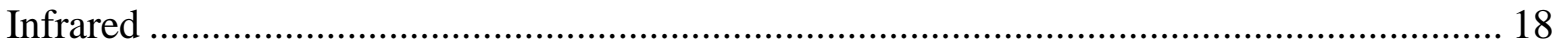

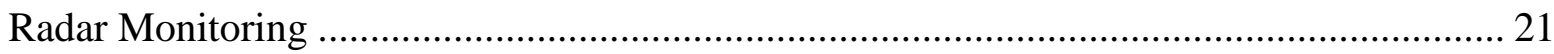

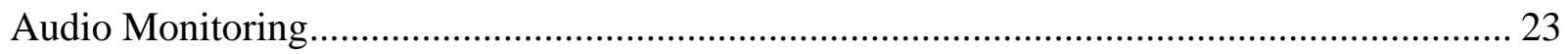

Carbon Dioxide Monitoring ……………………………........................................... 25

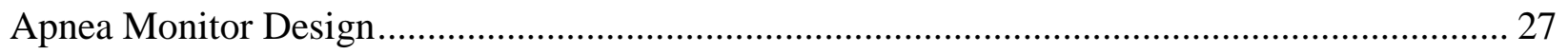

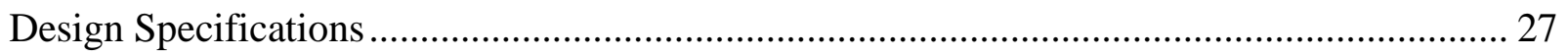




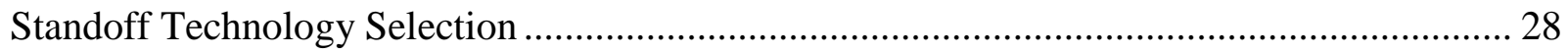

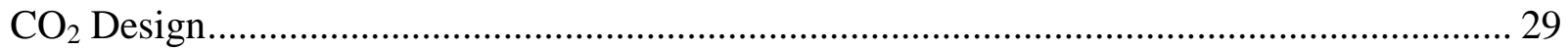

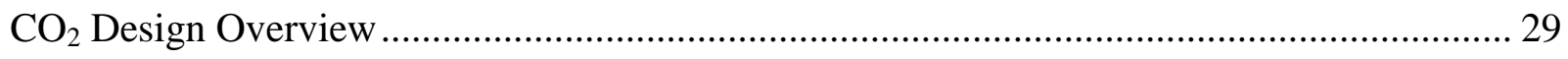

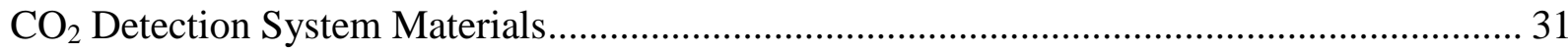

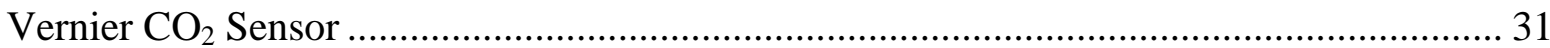

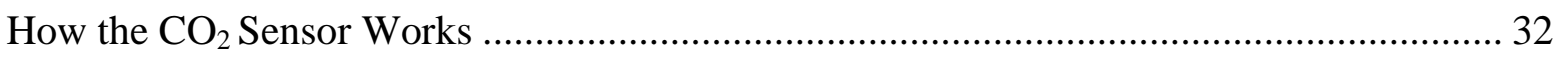

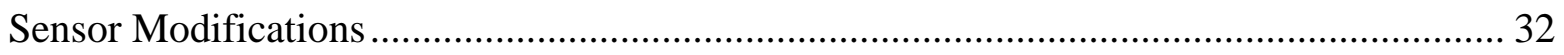

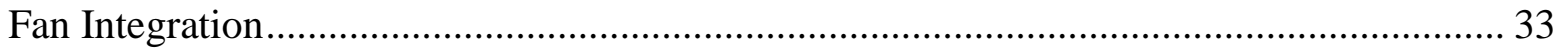

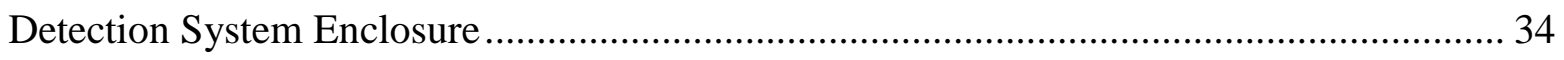

$\mathrm{CO}_{2}$ Apnea Detection Software .................................................................................... 35

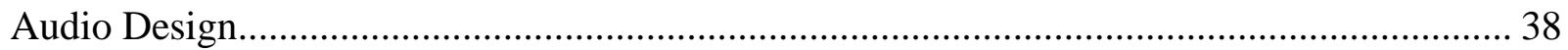

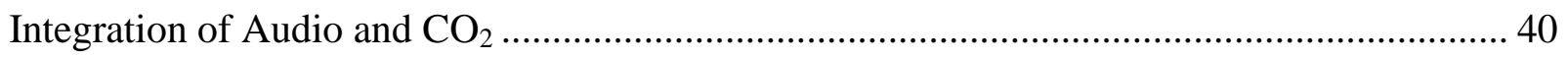

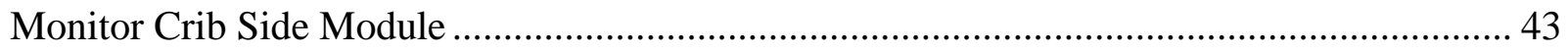

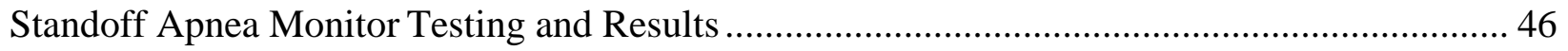

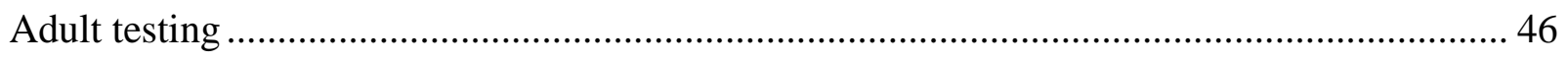

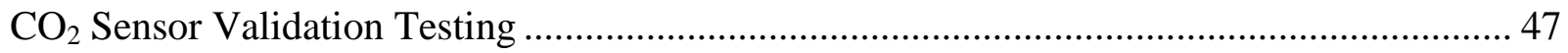

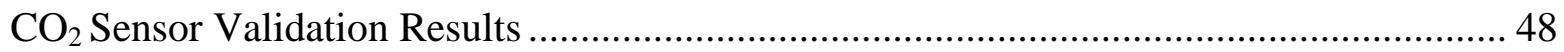

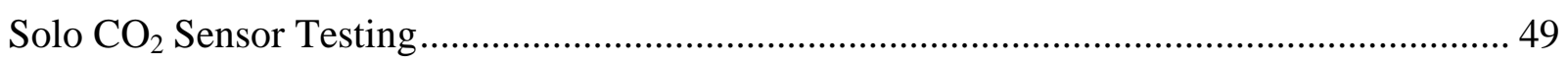

$\mathrm{CO}_{2}$ Sensor Results................................................................................................. 50

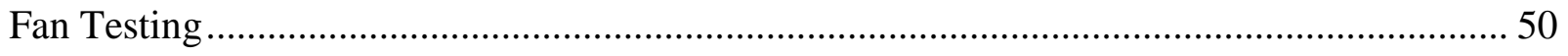

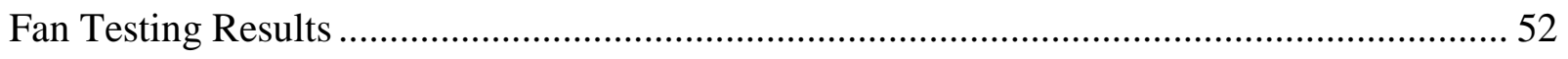

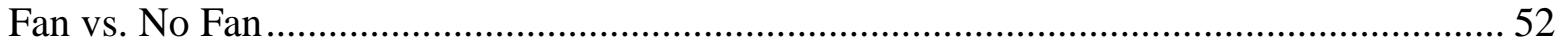

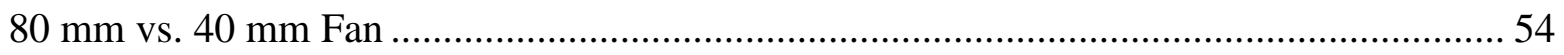

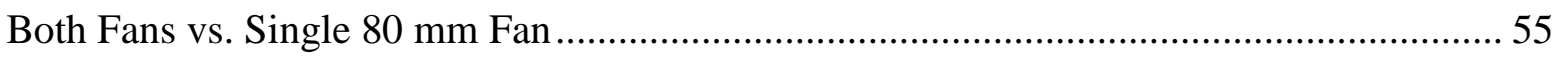

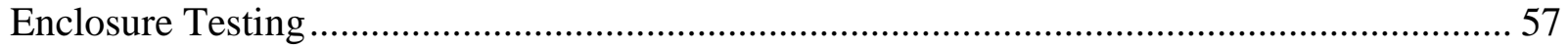

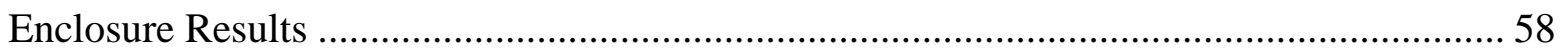

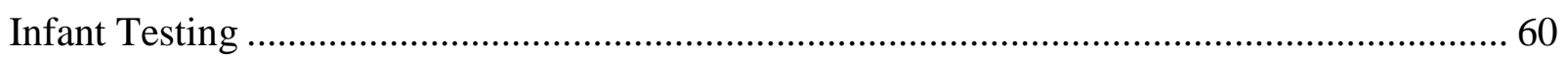

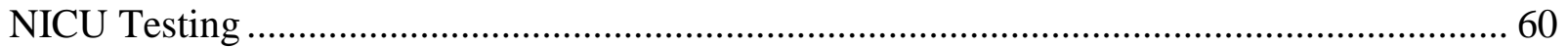

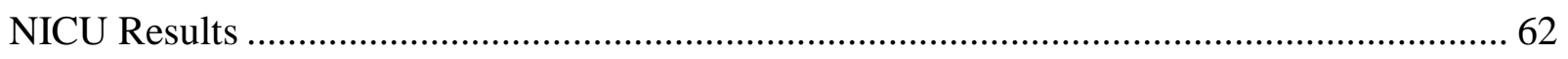

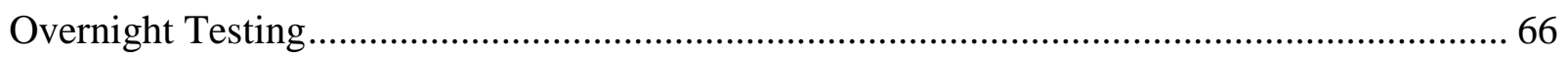

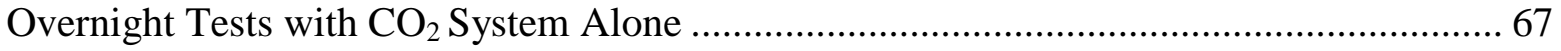




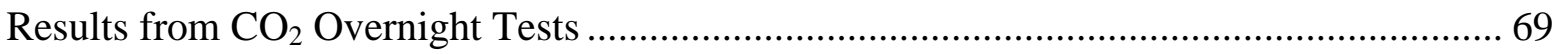

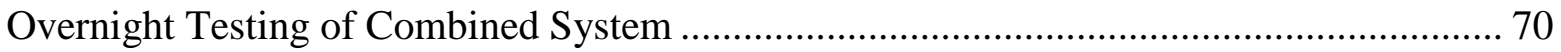

Results of Integrated Systems Testing.......................................................................... 71

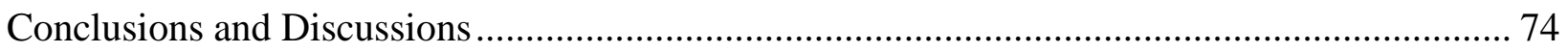

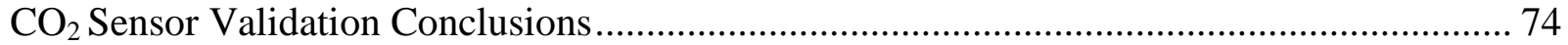

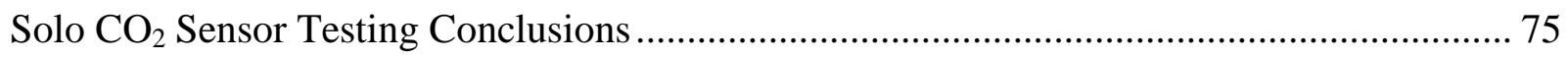

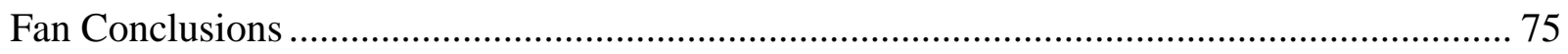

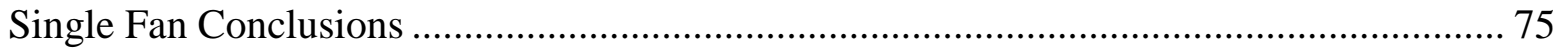

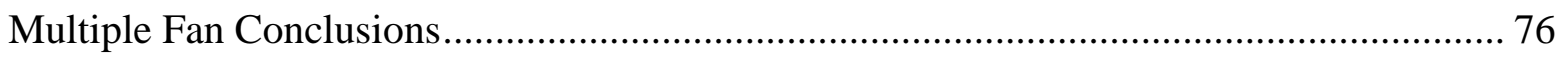

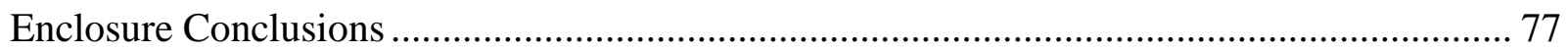

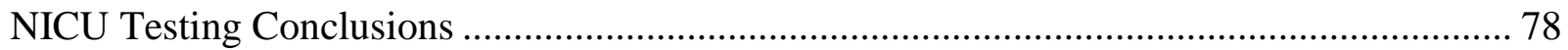

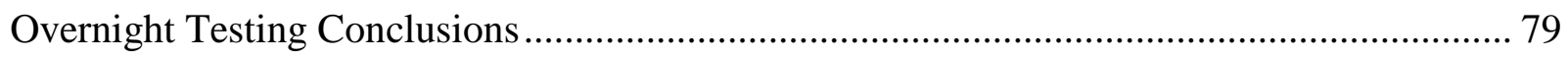

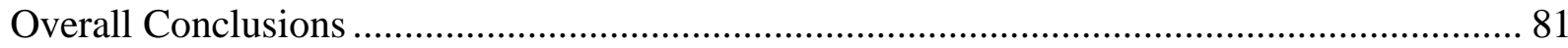

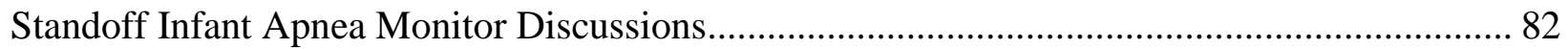

Standoff Technology Selection Discussion......................................................................... 82

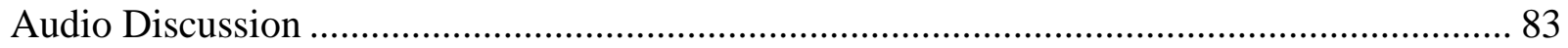

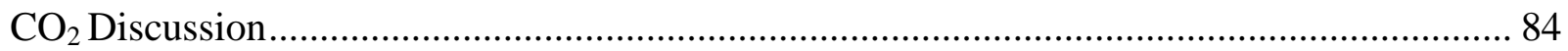

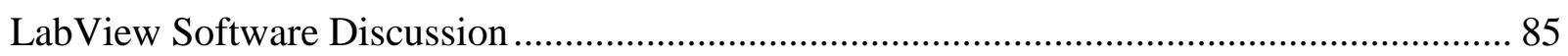

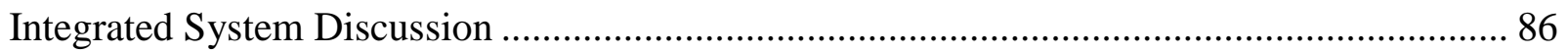

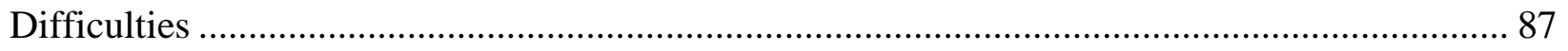

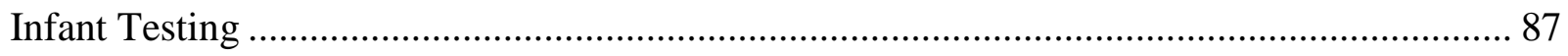

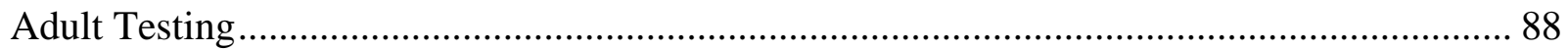

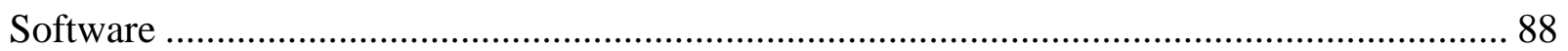

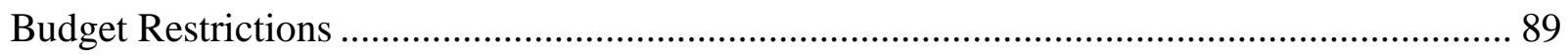

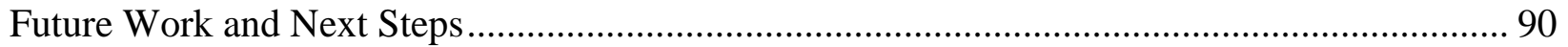

Continued Infant Testing.................................................................................................. 90

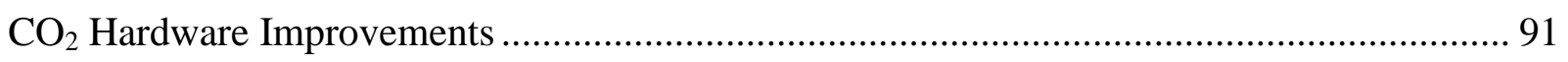

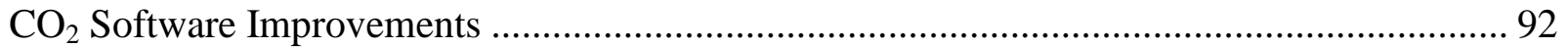

Other Applications of Researched Standoff Technologies ..................................................... 93

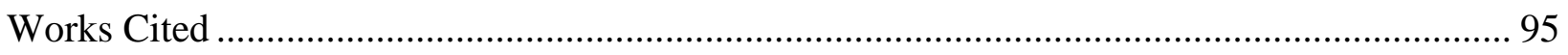




\section{List of Figures}

Figure 1 - Piezo-respiratory output of (a) quiet breathing and (b) after exercise [4]................... 3

Figure 2 - Oxygen and Carbon Dioxide gas exchange[3] ................................................ 5

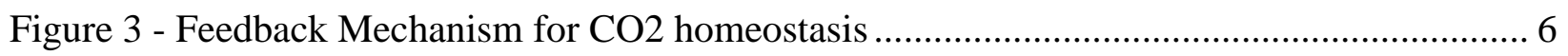

Figure 4 - Comparison of Infant/Child and Adult Upper Airway Anatomy [5] ........................ 7

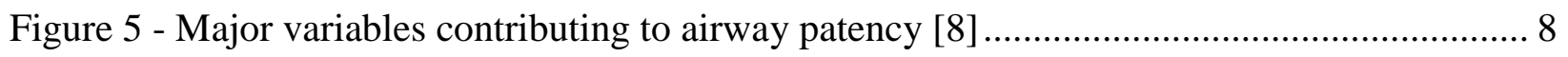

Figure 6 - Visual representation of Obstructive Apnea [11] .............................................. 11

Figure 7 - Examples of Infant Apnea Home Monitors [13] ............................................... 13

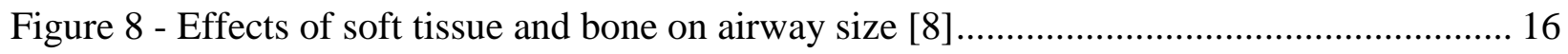

Figure 9 - Adult Sleep Apnea Monitoring Device [8] .................................................... 17

Figure 10 - Thermal imaging of multiple faces [19]..................................................... 19

Figure 11 - Visualization of Breath during nonexpiration and expiration [4] ......................... 20

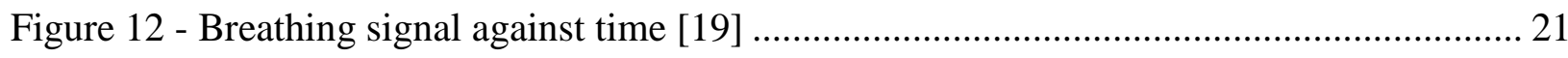

Figure 13 - Radar cardiogram of a subject using the RSVM technology[20] ......................... 22

Figure 14 - Experimental results of time-frequency analysis of wheezing [22] ...................... 24

Figure $15-\mathrm{CO}_{2}$ Apnea Detection System Purchased Hardware .......................................... 29

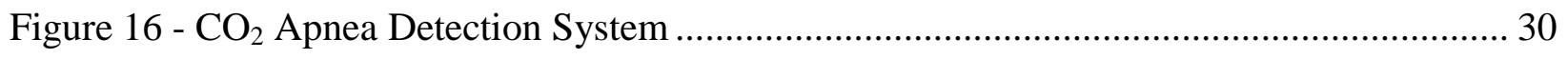

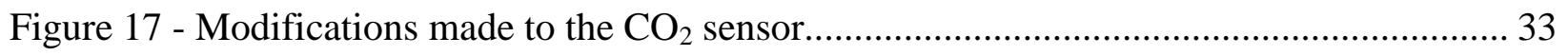

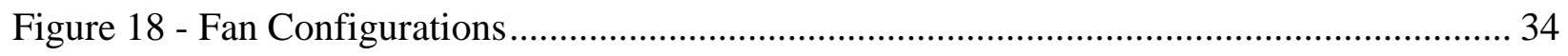

Figure $19-\mathrm{CO}_{2}$ Detection System Enclosure ............................................................ 35 
Figure 20 - Block diagram of $\mathrm{CO}_{2}$ detection system .

Figure 21 - Audio System Hardware 39

Figure 22 - Audio Detection System Front Panel ............................................................. 40

Figure 23 - Front Panel of Infant Apnea Monitoring System ............................................. 42

Figure 24 - LabView block diagram of integrated code ................................................. 43

Figure 25 - Standoff Infant Apnea Crib side Module ...................................................... 44

Figure 26 - Underside of Crib Side Module …................................................................. 45

Figure 27 - Visual description of orientation during fan testing ........................................ 52

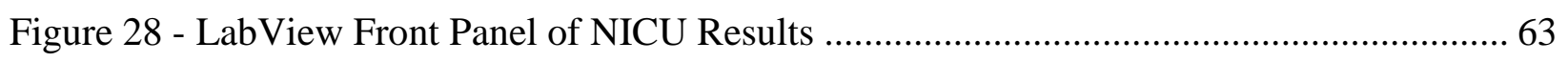

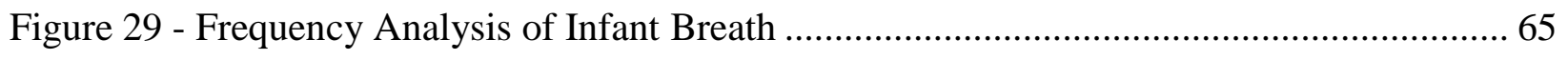




\section{List of Tables}

Table 1 - Comparison of neonatal, infant, and adult vital signs [5]......................................... 4

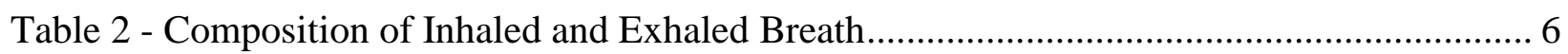

Table 3 - Comparison of Obstructive and Central Apnea in Adults [16] ................................... 15

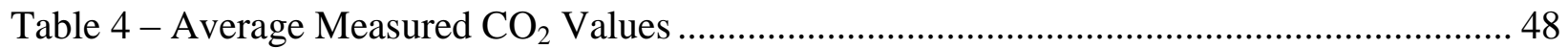

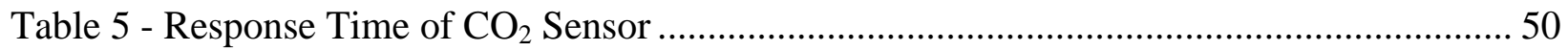

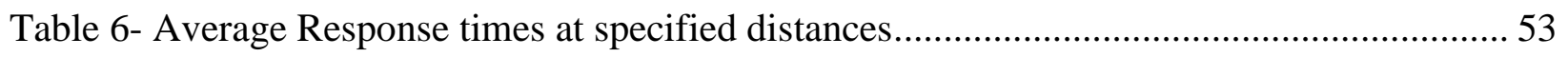

Table 7 - Average Response Times for both fans ........................................................................ 54

Table 8 - Results from Testing Both Fans vs. Single 80 mm Fan ................................................ 56

Table 9- Results from Enclosure Testing from One Foot............................................................ 58

Table 10 - Enclosure Response Time from Two Feet .................................................................... 59

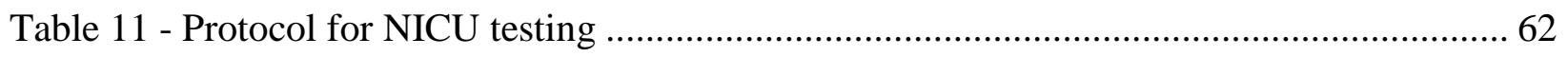

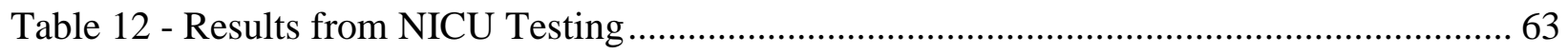

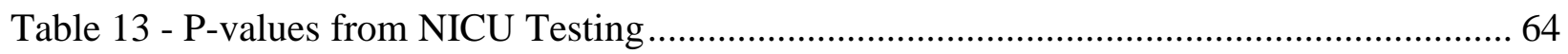

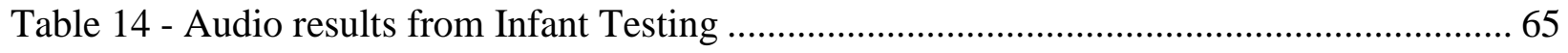

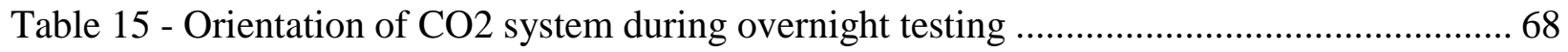

Table 16 - Data recorded from 15 overnight tests ...................................................................... 69

Table 17 - Logged Apnea Events for overnight testing .............................................................. 72

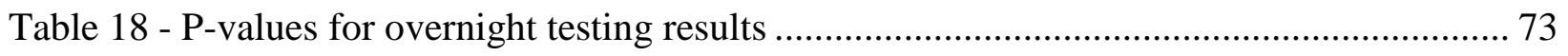




\section{List of Plots}

Plot 1 - Boxplot of Differences between measured and hypothesized $\mathrm{CO}_{2} \ldots \ldots \ldots \ldots \ldots \ldots \ldots \ldots \ldots . . . . . . . . . . . .49$

Plot 2 - Boxplot of response time with and without a fan ................................................. 54

Plot 3 - Boxplot of mean response time, in seconds, for each fan size …................................ 55

Plot 4 - Boxplot of Both Fans vs. Single 80 mm Fan ......................................................... 56

Plot 5 - Boxplot of Enclosure vs. No Enclosure Response Time ............................................. 59

Plot 6 - Boxplot of Enclosure Testing from Two Feet........................................................... 60

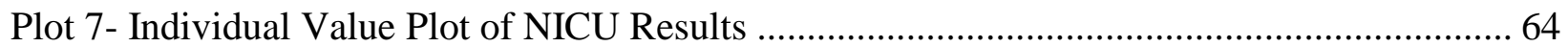

Plot 8 - Boxplot of Compared Orientations ....................................................................... 70

Plot 9 - Boxplot of Apnea Detection Systems ............................................................. 73 


\section{Introduction}

\section{Thesis Objective}

The objective of this thesis was to design, test, and evaluate a new system to detect infant apnea using standoff technologies. Standoff technologies require no contact with the subject being monitored. The end goal of this thesis was a proof-of-concept device that could detect infant breath without contact with the infant. The interests were in the standoff technologies and not in the final marketable crib side module.

To aid students who work on future generations of this device, the objective was also to state difficulties and future directions. Suggestions are made to improve the hardware and software of the monitor.

\section{Thesis Purpose}

The purpose of this thesis was 1) to research non-contact technologies to detect infant breath 2) select at least one technology to implement 3) design the hardware and software to detect breath using the selected technology 4) test the proof-of-concept standoff infant apnea monitor and 5) conclude if the chosen standoff technologies were capable of detecting infant breath.

Testing was conducted on both infants and adults. It was not feasible to run proof-ofconcept tests on infants with apnea, due to the availability of infants with apnea and hospital protocol, so the testing was conducted on healthy infants and adults without sleep apnea. The purpose of the infant testing was to show that the standoff infant apnea monitor could detect infant breath. The purpose of the adult testing was to implement design modifications, test simulated apnea events, and look at the rate of false apnea events detected, termed false alarms. 


\section{Background}

\section{Why Monitor Infant Breathing?}

Infant death is a tragedy no matter what the cause. This could be due to infant sleep apnea, Sudden Infant Death Syndrome (SIDS), or other preexisting conditions. Each year 4,500 infants die of SIDS in the US [1]. Although it is not known what causes SIDS, proper infant monitoring of "at risk" infants can help reduce this number. At risk infants can include premature infants and infants with preexisting cardio or pulmonary conditions. Many studies have been done to attempt to link SIDS and infant apnea, but there has been no scientific correlations made [2]. An estimated $\$ 24$ million dollars is spent on monitoring preterm and full term infants every year [2]. With parents willing to spend money to ensure their child's safety, the question becomes, which monitoring technique can accurately measure their child's breathing and not cause discomfort to the child. This thesis will focus on a way to monitor infant breathing from a standoff approach, so as to cause no discomfort to the infant while giving the parents or caregivers peace of mind, knowing their child is safe.

\section{Respiratory Physiology}

Pulmonary ventilation, or respiration, is a mechanical process that depends on volume changes in the thoracic cavity. Respiration consists of three defined stages. The first stage is termed breathing, which consists of inspiration of oxygen into the lungs and exhalation of air, rich in carbon dioxide. The second stage is the transport of the inhaled oxygen to tissues and cells, using the vascular system. The third stage is using that oxygen in the cells to generate energy for other physiologic activities. For the purpose of this thesis the focus was on the first stage of respiration, breathing [3]. 


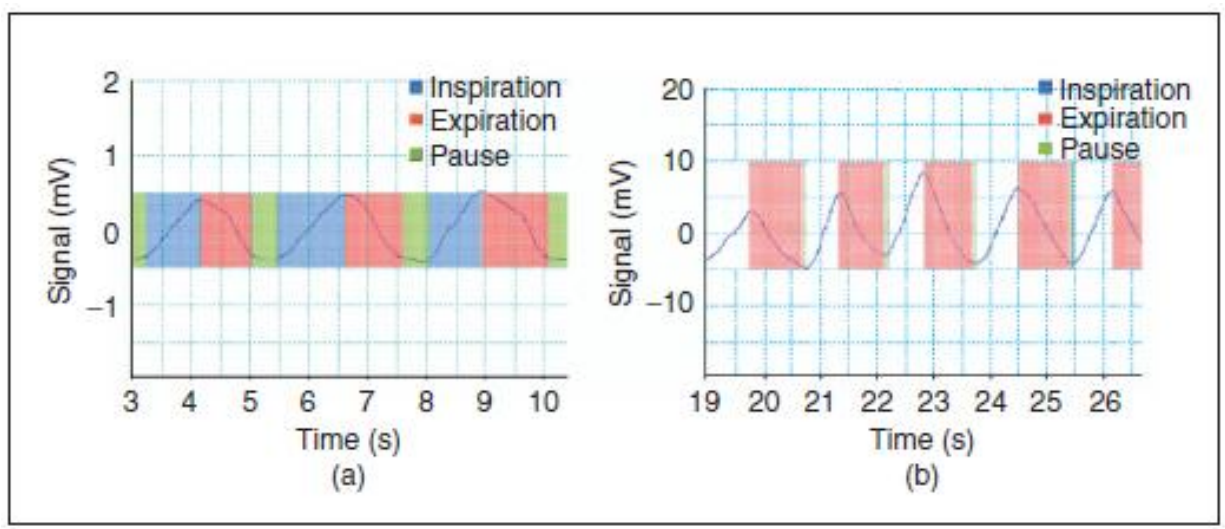

Figure 1 - Piezo-respiratory output of (a) quiet breathing and (b) after exercise [4]

The breathing cycle also consists of three phases, inhalation, expiration, and a postexpiratory pause. In Figure 1, the graph on the left shows the output from a piezo-electric belt transducer during quiet breathing. Blue represents inspiration, red represents exhalation, and green represents the post-expiratory pause. The graph on the right shows the same concept, but after exercise. The time of each breath is shortened and the peaks of the signal are higher due to greater exertion during breathing after exercise. A breathing cycle is defined as the time interval between the beginning of inspiration and the end of the postexpiratory pause [3].

Inspiration is due to negative pressure created inside the chest cavity. The inspiratory muscles contract causing the diaphragm to descend and the rib cage to rise. This increases the thoracic volume and the lungs expand increasing the intrapulmonary volume. The intrapulmonary pressure drops causing air to rush into the lungs, in the form of a breath, to equalize the pressure. Once the pressure is equalized, inspiration is complete [3].

Expiration is a passive process where the air flow occurs due to the elastic recoil properties of the lungs. As the inspiratory muscles relax back to resting length the rib cage, descends and the lungs recoil, decreasing the intrapulmonary volume. This compresses the 
alveoli forcing air out of the lungs in exhalation. The postexpiratory pause is caused when there is an equalization of the pressures inside the lungs and the atmosphere outside [4].

Table 1 - Comparison of neonatal, infant, and adult vital signs [5]

\begin{tabular}{|lc|}
\hline \multicolumn{2}{|c|}{ Neonatal vital signs (full-term, $\leq \mathbf{2 8}$ days) } \\
\hline Pulse & 120 to 160 beats per minute \\
Blood pressure & $>60 \mathrm{mmHg}$ systolic \\
Respirations & 40 to 60 breaths per minute \\
\hline Infant vital signs (age $\mathbf{1}$ to $\mathbf{1 2}$ months) \\
\hline Pulse & 100 to 140 beats per minute \\
Blood pressure & 70 to $95 \mathrm{mmHg}$ systolic \\
Respirations & 25 to 50 breaths per minute \\
\hline Adult vital signs & \\
\hline Pulse & 60 to 100 beats per minute \\
Blood pressure & 90 to $140 \mathrm{mmHg}$ (systolic) \\
& 60 to $90 \mathrm{mmHg}$ (diastolic) \\
Respirations & 12 to 20 breaths per minute \\
\hline
\end{tabular}

Table 1 shows a comparison of neonatal, infant, and adult vital signs. For this thesis, it is important to note the respiration rates. For a neonatal, full-term infant ( $\leq 28$ days), a normal breathing rate may vary from 40-60 breaths per minute. For an infant, ages $1-12$ months, a normal breathing rate may vary from 25-50 breaths per minute. For an average adult, during quiet breathing, the breathing rate may vary from 12-20 breaths per minute and after physical activity, 30-40 breaths per minute [5].

\section{Carbon Dioxide}

This section will cover the physiologic processes that require carbon dioxide. Carbon dioxide plays a role in oxygen exchange in the lungs and capillaries. It is also the prominent molecule responsible for respiration. Both of these processes are described at length in this section. 


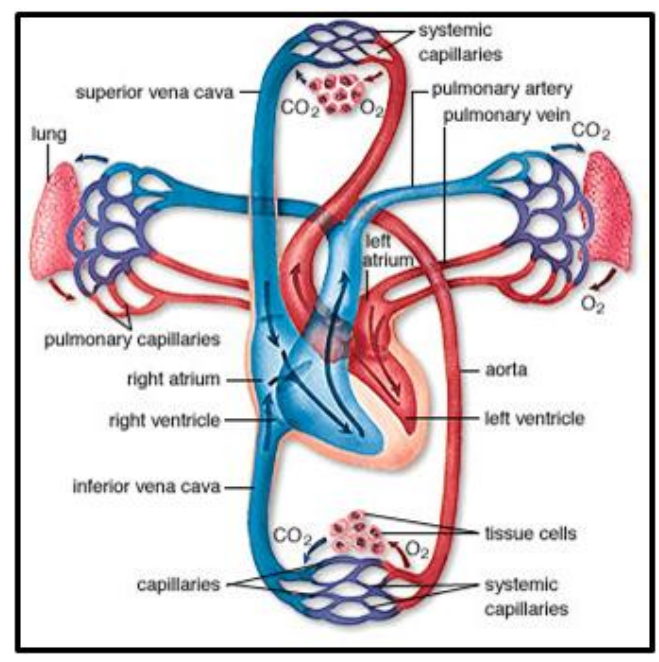

Figure 2 - Oxygen and Carbon Dioxide gas exchange[3]

As humans, we inhale oxygen and exhale carbon dioxide. During gas exchange in the lungs, oxygen diffuses from the alveoli to capillaries while carbon dioxide moves in the opposite direction. Figure 2 displays the oxygen input and carbon dioxide output of both the lungs and the systemic capillaries. At the center of the figure is the heart that takes in deoxygenated blood through the right side from the rest of the body and pumps it into the lungs. The lungs then take up $\mathrm{CO}_{2}$ from the blood and oxygenate the blood. We then exhale that $\mathrm{CO}_{2}$, along with other gases, into the atmosphere. There is some $\mathrm{CO}_{2}$ and oxygen exchange in the capillary beds throughout our body.

Of all the chemicals responsible for respiration, $\mathrm{CO}_{2}$ is the most closely controlled and the most potent [3]. Normal arterial $\mathrm{CO}_{2}$ pressure $\left(\mathrm{P}_{\mathrm{CO} 2}\right)$ is maintained at $40 \mathrm{mmHg} \pm 3 \mathrm{mmHg}$. Carbon dioxide can easily diffuse from blood into the cerebrospinal fluid (CSF). From there a series of chemical reactions turns the $\mathrm{CO}_{2}$ into carbonic acid. This causes the $\mathrm{pH}$ in the CSF to drop, which triggers the chemoreceptors in the medullary respiratory center. The chemoreceptors trigger the respiratory muscles and ventilation is increased. Increased ventilation results in more $\mathrm{CO}_{2}$ being exhaled which raises the $\mathrm{pH}$ in the CSF back to normal levels [3]. Figure 3 is a 
pictorial representation of the process described above. It transitions from the stimulus on the left to the result on the right.

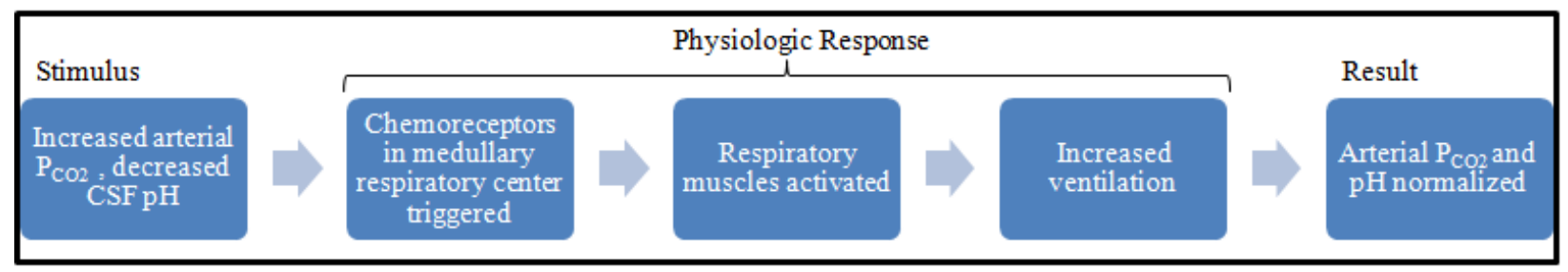

Figure 3 - Feedback Mechanism for CO2 homeostasis

The air that we inhale and exhale is composed of different molecules and different concentrations. Table 2 shows a comparison of the composition of inhaled air and exhaled air. Inhaled air has a higher percentage of oxygen than exhaled air. Exhaled air has a much higher percentage of carbon dioxide than inhaled air, making it a good measure for actual breath. The percentage of $\mathrm{CO}_{2}$ that is exhaled is 125 times greater than what is inhaled.

Table 2 - Composition of Inhaled and Exhaled Breath

\begin{tabular}{|c|c|c|}
\hline & Inhaled Air (\%) & Exhaled Air (\%) \\
\hline Oxygen & 21 & 17 \\
\hline Nitrogen & 78 & 78 \\
\hline Inert Gas & $<1$ & $<1$ \\
\hline Carbon Dioxide & 0.04 & $\sim 5$ \\
\hline Water Vapor & little & saturated \\
\hline
\end{tabular}

\section{Infant Respiratory Anatomy}

The upper airway, consisting of the nose, mouth, pharynx, and larynx, is an essential component of the breathing apparatus and exerts an important influence on breathing from the fetal developmental period onward. These structures participate in lung development, they are 
responsible for establishing optimal end expiratory volume, and decrease upper airway inspiratory resistance during breathing. Anatomical abnormalities in these structures can lead to upper airway dysfunction and obstructed breathing in newborns. Neural immaturity in a newborn is often responsible for reflexes originating from the nose or the laryngeal region, which are inhibitory to cardio-respiratory rhythm [6].

An infant's upper airway cannot be considered a miniaturized version of an adult upper airway. When babies are born they have a different anatomical structure of the area around their larynx than adults do. Figure 4 shows the anatomical structure of an infant's upper airway compared to that of an adult. The important differences between the two are: the infant's tongue is larger in proportion to their mouth, the pharynx of the infant is smaller than that of the adult, the larynx of an infant is also more anterior and superior in reference to the tongue, and the trachea is narrower and less rigid.

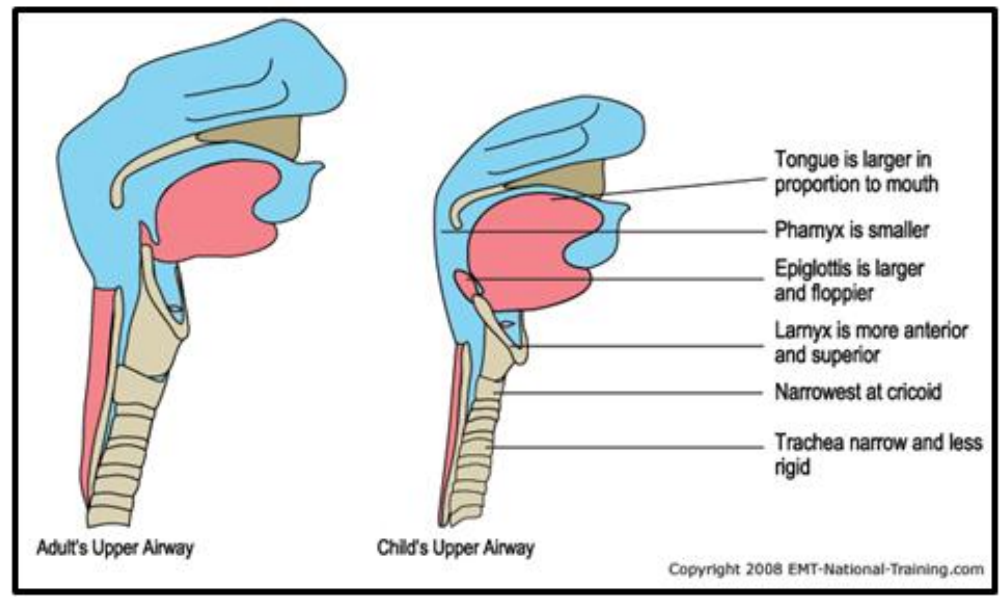

Figure 4 - Comparison of Infant/Child and Adult Upper Airway Anatomy [5]

An infant's soft palate is in direct contact with their epiglottis; which allows an infant to swallow liquid and breathe at the same time. The liquid passes through the isthmus faucium to the pharynx on both sides of the elevated larynx. The term used to describe this ability to breathe 
and swallow at the same time is termed "obligate nose breathing." Infants are obligate nose breathers until around 5 months of age [6].

Infants have a much smaller lung volume capacity than adults. Infants, on average, take 25-50 breaths per minute. Each inhalation and exhalation contains about $15 \mathrm{ml}$ of air. This equals out to about 0.5 liters per minute [7]. This means that the volume of exhaled carbon dioxide is very small and more difficult to monitor.

\section{Adult Respiratory Anatomy}

While the anatomical structures do not change between infancy and adulthood, they do grow and mature. This section will discuss the maturity of the upper airway into adulthood. Refer to the previous section and Figure 4 to see how the upper airway structures differ in an infant. The diameter of the adult trachea is larger allowing large volumes of gas exchange to take place. In humans, the upper airway, from the end of the nasal septum to the epiglottis, has relatively little bony or rigid support. The two primary forces that tend to collapse the airway are the intraluminal negative pressure generated by the diaphragm during inspiration and the extraluminal tissue pressure. These muscles surrounding these structures have to counteract these forces [8]. This is visually represented in Figure 5.

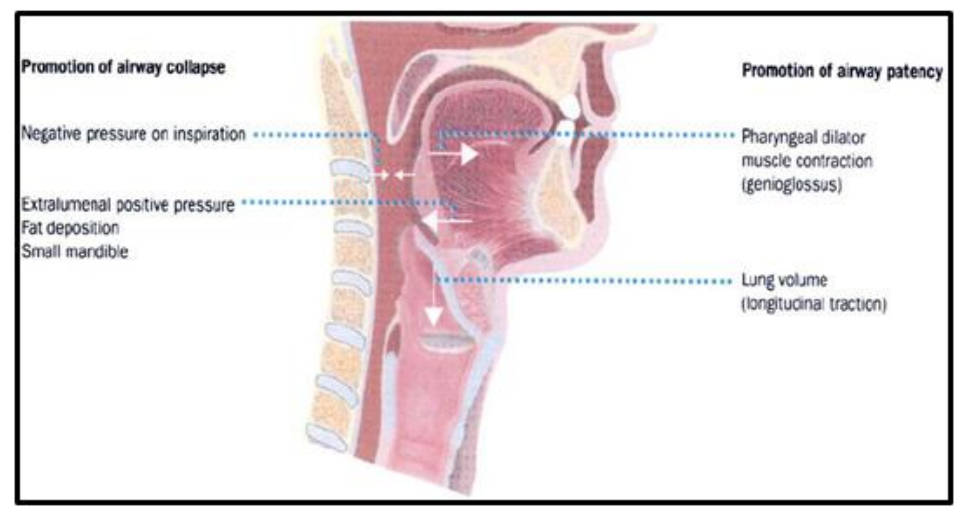

Figure 5 - Major variables contributing to airway patency [8] 
When looking at respiration rate, there is a considerable difference between infant and adult. Adults take anywhere from 12 to 20 breaths per minute. Adults also have a much larger lung volume capacity than infants. Adult males have a total lung capacity of 6 liters. The average amount of air inspired or expired in one adult breath is $500 \mathrm{ml}$. These rates need to be observed when discussing apneas and techniques to monitor them.

\section{Apnea}

The following sections will discuss both infant apnea as well as adult apnea. The upper respiratory anatomy discussed in the previous sections will be an important foundation for the following sections. Apnea is defined as a cessation of breathing for ten seconds or longer. Both infant and adult apnea, have subcategories of central and obstructive apnea. There are differences when it comes to monitoring apnea in infants and adults. These similarities and differences will be explored in detail in the following sections.

\section{Infant Apnea}

Infant apnea is grouped into central apneas, obstructive apneas, or mixed that occur while the infant is asleep. An infant apnea event is defined as "an unexplained episode of cessation of breathing lasting at least 20 seconds, or a shorter respiratory pause associated with bradycardia (slow heart rate), cyanosis (turning blue), pallor (pale skin), and/or marked hypotonia (lack of muscle tone) [2]." Infant apnea generally refers to infants with gestational age of 37 weeks or more at the onset of apnea. Apnea of prematurity refers to gestational age less than 37 weeks [2]. For the purposes of this thesis, apnea of prematurity will not be discussed. Central apnea and obstructive apnea will be explored in detail. 


\section{Central Apnea}

Central apnea occurs when the part of the brain that controls breathing does not properly maintain the breathing cycle. In premature infants, it is not rare because the respiratory center in the brain has not fully developed. From birth to about five months of age an infant's brain is still in the primitive stage. Tasks, such as breathing, are involuntary and occur without input from the infant. Before the age of five months responses to stimulus are not learned [9]. This is important because if the infant is face down on the mattress, or a blanket falls over their face, they do not have the learned response to roll over and remove the blanket so they will not suffocate. Around five months an infant's responses transitions from primitive to learned, which corresponds to a change in brain activity. When looking at this phenomenon alongside infant apnea, they are closely correlated. Infant apnea seems to spike around five months and exponentially decrease after that. There is still a lot of research that needs to be done to correlate the two. Currently, no conclusive data exists to support this theory.

\section{Obstructive Apnea}

An obstructive apnea is when the upper airway is blocked. A common type of apnea in children, obstructive apnea is caused by an obstruction of the airway, such as swollen tonsils. When the infant is asleep, the soft tissue in the back of the throat is relaxed, making this the most likely time for obstructive apnea to occur [10]. Figure 6 shows the profile of the upper airway lying down. The soft tissue and tongue are relaxed and pressed up against the back of the throat, not allowing air to pass through into the lungs. 


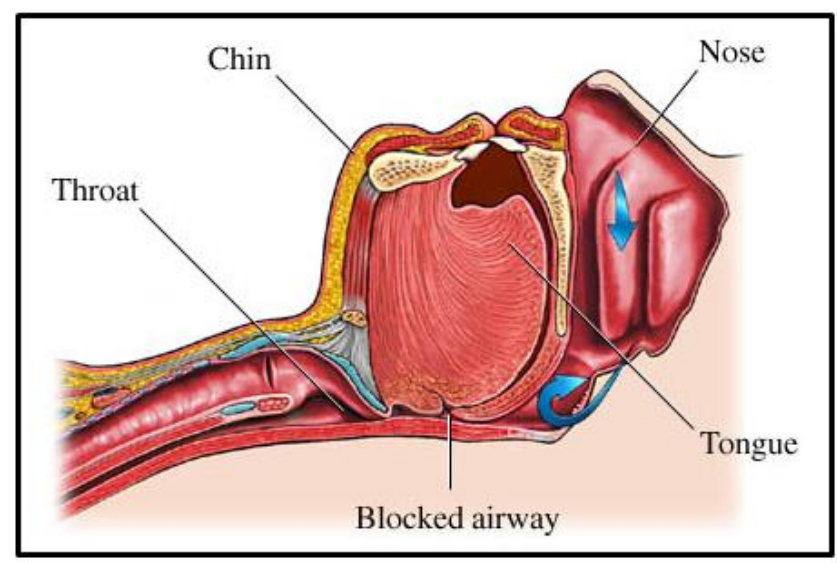

Figure 6 - Visual representation of Obstructive Apnea [11]

Outside of congenital abnormalities, there are two major causes of obstructive apneas. The most frequently recognized mechanism is passive pharyngeal collapse during inspiration. This can be due to cervical flexion, nose obstruction, or lack of pharyngeal muscle-diaphragm coordination. The second mechanism is active glottal closure which can be triggered by laryngeal chemoreceptors. The chemoreceptors can be triggered by things like saliva or liquid [6].

\section{Hospital Monitoring}

This section will discuss the current technologies that hospitals use to monitor infants for apnea. Apnea monitors were first introduced in the 1960's for managing apnea events in premature infants in a hospital setting [2]. The monitoring can either be done on premature infants as a precautionary measure or on infants who have displays apnea symptoms that are described in the previous section titled "Infant Apnea."

The best indications of an infant having an apnea episode are respiration rate, oxygen saturation, and heart rate. In a hospital setting there is not one device that monitors infant apnea. If an infant is being monitored for apnea, the doctor will hook the infant up to a monitor that will 
monitor heart rate, respiration rate, and oxygen saturation rate. This involves attaching several leads to the infant's chest and taping a pulse oximeter to the infant's foot. This can be uncomfortable for the infant as well as the new parent seeing their child like this. Full term infants and premature infants have very delicate skin. Placing multiple leads and tape on their skin can lead to further damage, such as a rash or even a burn from the tape being repetitively pulled off and put back on. This equipment is very accurate at detecting the slightest changes in signals. To understand the output of the equipment a physician's knowledge is required. This type of monitoring is only done in a NICU setting. Once the doctor feels that the infant can be sent home, they can either give the parents a monitor to take with them or the parents can purchase a monitor over the counter. The following section will discuss the advantages and disadvantages of infant apnea home monitoring systems.

\section{Home Monitoring}

This section will discuss the current technologies that are used to monitor infant apnea in a home setting. It will review a study done on infant apnea monitors. It will also discuss the disadvantages to the current monitoring technologies.

Infants with apnea can be observed at home with the help of a special cardiorespiratory monitor prescribed by a sleep specialist [2]. This monitor records chest movements and/or heart rate and can relay the readings to a hospital apnea program or save them for future evaluation by

a physician. Before sending the child home, parents and caregivers should be taught CPR, in case an emergency arises. If the infant stops breathing due to infant apnea, the parents only have a couple of minutes to become aware and do something about it. If they get to the child in time they can revive the infant. Sometimes all the baby needs is stimulation, such as rubbing their feet 
or chest, for the infant to resume normal breathing. Other times, CPR might be necessary and in some cases professional emergency care is necessary.

One study found that the number one problem with at home monitoring equipment was the rate of false alarms [12]. A total of 236 mothers were approached for an at home monitoring study and only 53 were recruited to participate. Some parents were not satisfied with the monitor, and $15 \%$ of those participating in the study returned the systems due to multiple false alarms. Once all the data was collected from the monitors, 534 of the 728 alarm events were found to be false alarms [12]. This correlates to a $73 \%$ false alarm rate. The other conclusion from this study was the need for more than one biosignal, such as both heart rate and respiration rate.

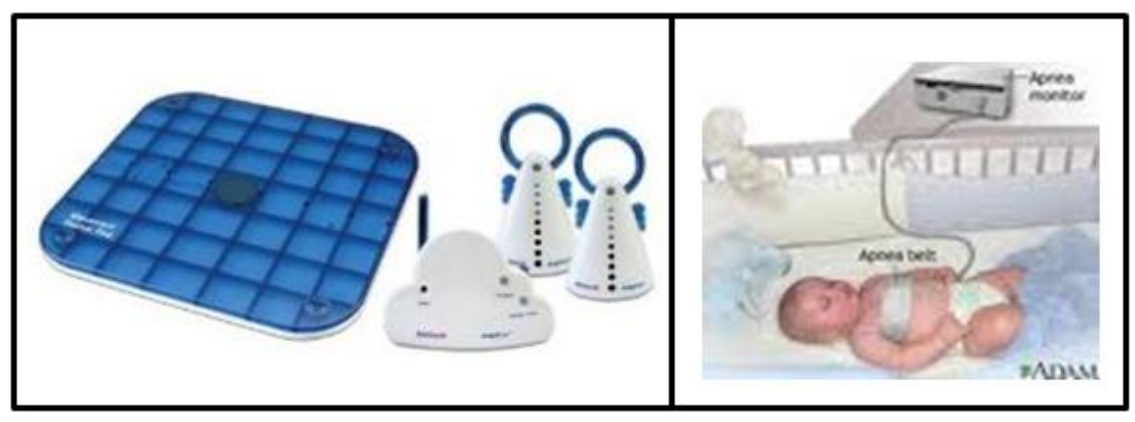

Figure 7 - Examples of Infant Apnea Home Monitors [13]

Parents can also purchase infant apnea monitoring systems at local retailers, such as the ones seen in Figure 7. These include a pad that can be placed under the infant's mattress to detect chest vibrations and a chest strap that monitor chest rise and fall. There are also small monitors that clip onto a child's diaper and detect movement. If no movement is detected for 20 seconds the parents are alerted [14]. These monitors can range upwards of 300 dollars to purchase online or in a store. All of these monitors use vibrations during chest movement to track apnea events. If the infant stops breathing and chest movement also ceases, these monitors can be useful. Chest 
rise and fall is not always correlated to the cessation of breathing [15]. The infant's chest can be moving but they are struggling for breath and the monitor will miss the event.

All monitors should record at least two different biosignals, such as respiration rate and heart rate. The percentage of detected apnea events can increase greatly with the use of a second technology. The false alarm rate would also diminish [12]. Many of these over the counter monitoring devices do not allow you to revisit a log from the previous night to see if and when apnea events occurred. This can be useful information for a physician. Our device will alert the parents within seconds of the apnea event and give them those precious few minutes to act on behalf of their child. Our goal is to give parents the peace of mind that should something go wrong and their baby have an apnea event, they will be alerted within seconds and be able to respond in time.

\section{Adult Sleep Apnea}

This section will discuss adult sleep apnea. There are similarities and difference to infant apnea that will be discussed. This thesis was focused on detecting infant apnea, but there are applications of the monitor for adult sleep apnea. Much of the testing in this thesis was conducted on adults, therefore it important to present the issue that surround adult apnea.

Adult sleep apnea is similar to infant apnea in that it is the cessation of breathing while asleep and there is both central and obstructive apneas. The main difference between central and obstructive apnea is that obstructive apnea deals with a blockage of the airway and central deals with an unstable ventilatory control system [8]. Table 3 displays the two types of sleep apnea, a description of the problem, where the problem occurs, and a few of the major causes. These will be discussed in detail in the following sections. 
Table 3 - Comparison of Obstructive and Central Apnea in Adults [16]

\begin{tabular}{|c|c|c|c|}
\hline $\begin{array}{c}\text { Type of Sleep } \\
\text { Apnea }\end{array}$ & The Problem & Site of Problem & Major Causes \\
\hline Obstructive & Airflow is blocked & Airway & Weight \\
Air cannot enter lungs & & $\begin{array}{c}\text { Tonsils } \\
\text { Jaw anatomy }\end{array}$ \\
\hline Central & $\begin{array}{c}\text { The drive to breathe is } \\
\text { reduced }\end{array}$ & Brain & Heart failure \\
\hline
\end{tabular}

\section{Central Apnea}

The human body uses sets of feedback loops, such as chemoreceptors, intrapulmonary receptors, and respiratory muscle afferents to maintain oxygen and carbon dioxide levels. These loops are working during the wake cycle and the sleep cycle, although the behavioral influences are only present while we are awake [8]. For a patient with central apnea these feedback loops are not working properly. This could be due to a number of conditions, including cardiovascular disease. There is a delay between the lungs and the $\mathrm{CO}_{2}$ sensors in the carotid body and brainstem. This delay is amplified in patients with congestive heart failure [16].

\section{Obstructive Apnea}

Adult obstructive sleep apnea results when the upper airway anatomy collapses, periodically blocking air during inspiration [11]. There is also thought to be a neuromuscular component to obstructive apnea. Most obstructive apnea patients have an anatomically small upper airway due to either increased soft tissue surrounding the airway or a small bony compartment in which the airway is enclosed. While awake, the pharyngeal dilator muscle activation is responsible for maintaining patency. While asleep, the reflex driven, muscle activation is reduced [8]. A major cause of obstructive apnea is obesity, which increases the soft 
tissue in the already confined pharyngeal space. Increased weight also requires your diaphragm and heart muscle to work harder to properly exchange oxygen and carbon dioxide [16].

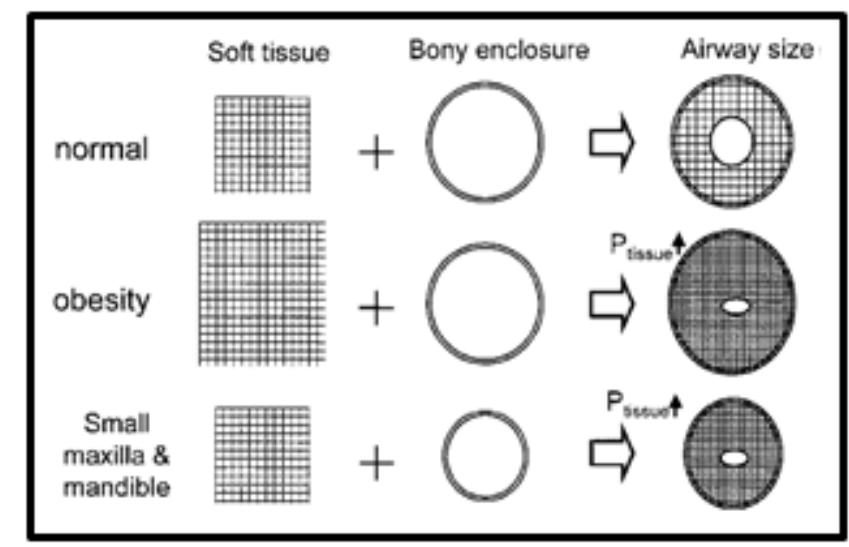

Figure 8 - Effects of soft tissue and bone on airway size [8]

Figure 8 shows how some of the causes of obstructive sleep apnea affect the size of the airway. Airway size has a direct effect on the amount of air that can be passed into and out of the lungs. Obesity causes an excess of soft tissue in the same size bony structure as a normal upper airway, causing the need for increased pressure to move air through the airway. Another cause of obstructive sleep apnea could be small bones around the airway, such as the maxilla and the mandible. This require the same amount of soft tissue as a normal airway to fit into a smaller bony structure, having the same decreased affect in the patency of the airway as obesity.

\section{Adult Home Monitoring}

If apnea is suspected, patients will undergo a sleep study at either a hospital or sleep clinic. During the study the patient is hooked up to chest and abdominal straps to monitor movement during breathing, a pulse oximeter to measure oxygen saturation, and leads to monitor heart rate and respiration rate. All of this equipment can be very cumbersome and make the patient uncomfortable and possibly compromise the study. A non-contact monitoring system 
would allow the clinicians to monitor the patient in a much more realistic setting and obtain more meaningful data.

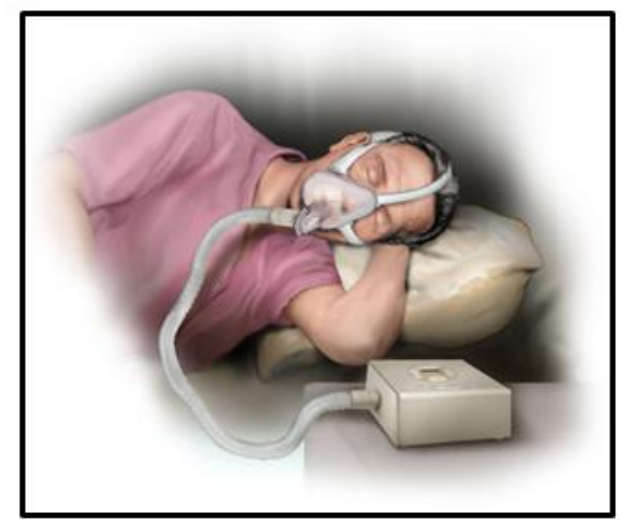

Figure 9 - Adult Sleep Apnea Monitoring Device [8]

Once a patient is diagnosed with sleep apnea, the doctor will usually prescribe a sleep apnea monitor, as seen in Figure 9. The most common treatment for sleep apnea is CPAP, or Continuous Positive Airway Pressure, therapy. The patient will have to wear a mask that sits over the nose and mouth and is attached around the back of the head. The mask will be connected to a bedside monitor with a flexible tube. CPAP works by pushing air through the airway passage at a pressure high enough to prevent apneas and can be prescribed for both obstructive and central sleep apnea. The pressure is set according to the patient's sleep apnea.

\section{Sudden Infant Death Syndrome (SIDS)}

Although it was already stated that there is no conclusive correlation between infant apnea and SIDS, it is important to explore this area. There are studies that show that there are ways to reduce the risk of SIDS and the device designed in this thesis could also help prevent infant deaths due to SIDS.

As of 1989, the definition of SIDS is "the sudden, unexpected death of an apparently healthy infant, less than one year of age, which remains unexplained after a thorough post- 
mortem investigation [17]. There has been a lot of research done around the causes of SIDS. A 2008 study looked at 497 infants in 11 California counties. They looked at 185 infant deaths with a SIDS diagnosis and 312 healthy infants as controls. Their results showed a $72 \%$ reduction of SIDS risk with a fan circulating the air around the crib. They attributed this to exhaled carbon dioxide that gets trapped in blankets and can concentrate around the infant's mouth, which is then inhaled again by the infant. Infants have a small circulatory and pulmonary system, so it does not take a lot of carbon dioxide to be detrimental and even fatal. Their conclusion was that a fan may be an effective intervention for decreasing the SIDS risk [18].

Researchers have looked very closely for a correlation between infant apnea and SIDS. While infant apnea can be fatal, death is not always the outcome and there has been no scientific correlation between infant apnea and SIDS [2]. The study discussed would suggest that monitoring $\mathrm{CO}_{2}$ levels around the infant may help reduce the rate of SIDS.

\section{Standoff Monitoring Technologies}

This section discusses the standoff technologies that were researched for this thesis. Standoff technologies monitor targets from a distance without contact with the target [4]. Most vital monitoring systems require some contact with the patient, either external with skin or more direct contact internally with the physiologic system of interest. By taking a complete standoff approach, we are limited in the technologies we can use. We chose to look at Infrared (IR) thermal imaging, radar monitoring, $\mathrm{CO}_{2}$ monitoring, and the audio signatures of a breath.

\section{Infrared}

The first technology that was researched for standoff monitoring applications was IR thermal imaging. Research has been done to evaluate the effectiveness of infrared imaging to 
monitor breathing. This technology is based on the fact that exhaled air has a higher temperature than the typical background environment [19]. Zhu et al. used the periorbital region, the two areas between the bridge of the nose, and the inner corner of the eyes, to monitor using infrared [19]. Figure 10 shows the regions that were focused on. The different shades of gray indicate different temperatures. The darker areas around the nose indicate colder temperature than around the eyes. The multiple pictures show that the areas look relatively the same from person to person making tracking different people feasible.

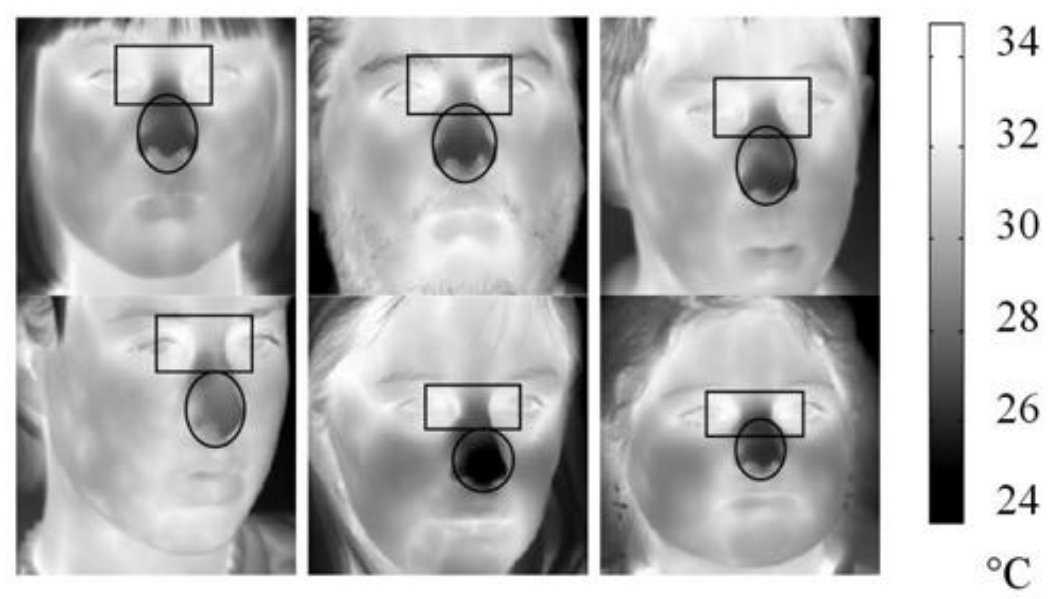

Figure 10 - Thermal imaging of multiple faces [19]

Using complex computer algorithms developed specifically for this application, they were able to track these features in the Measurement Region of Interest (MROI), which consists of the region below the nose and is expected to have the strongest breath signal [19]. The MROI can be seen in Figure 11. The small yellow rectangle under the nose is the MROI. The picture on the left is before expiration and no air is seen in the MROI. The picture on the right is after expiration. The exhaled air, in red, can be seen passing through the MROI, which would be picked up by the software. 


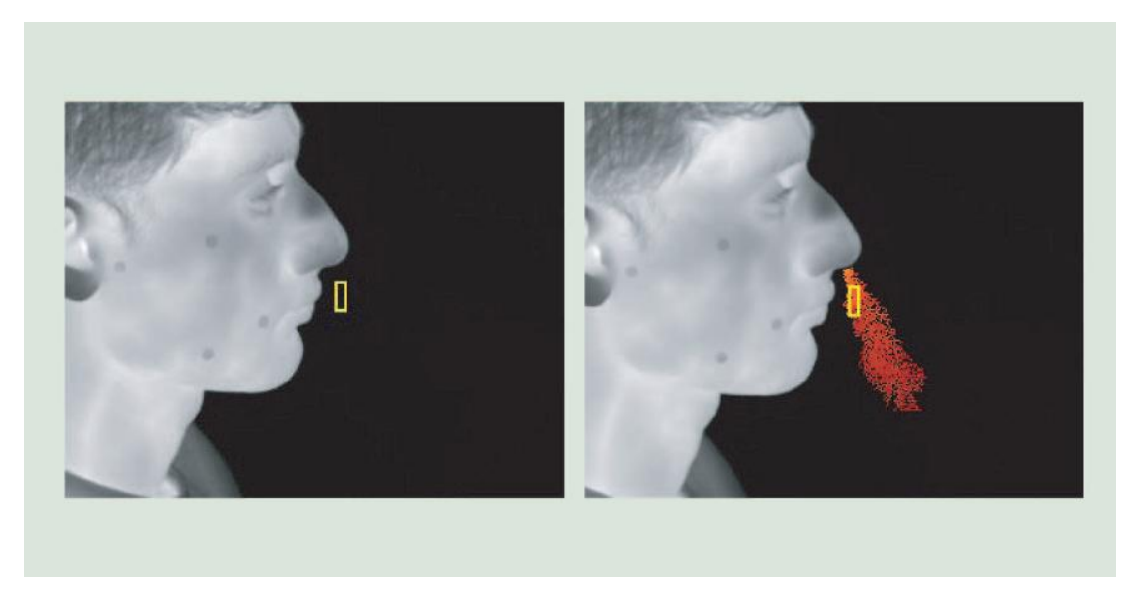

Figure 11 - Visualization of Breath during nonexpiration and expiration [4]

Once the MROI was defined, a computer algorithm was used to record and track the breathing. The tracking framework is particle filtering, a Monte Carlo method for Bayesian tracking. They imposed geometric and displacement constraints on the windows of the Tracked Region of Interest (TROI) and designed a measurement model in particle filtering, which makes the tracker perform reliably under different head poses and when part of the TROI is occluded. Video clips were captured using the highly automated IR image processing platform, ATHEMOS. The image is taken by adding a $4.3 \mu \mathrm{m}$ narrow band filter on the camera lens to visualize $\mathrm{CO}_{2}$ airflow [19].

Their experiments showed that the specially designed measurement model can prevent a number of tracking failures. Figure 12 shows the results of the model tracking each breathing cycle. The shaded bars represent the inspiration cycle, in which the temperature dropped due to the lack of $\mathrm{CO}_{2}$ in the MROI. The white sections represent expiration when the $\mathrm{CO}_{2}$ is exhaled and the temperature in the MROI increases. 


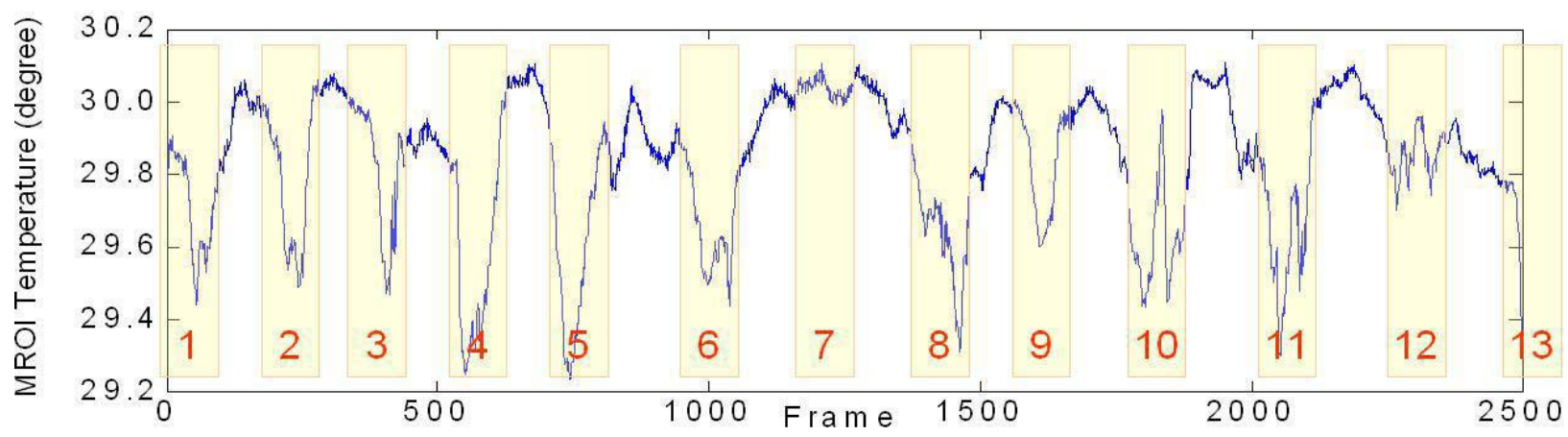

Figure 12 - Breathing signal against time [19]

This standoff technology had a few disadvantages when looking at applying it to our scenario of monitoring infants. The cameras required to obtain the accuracy seen above, can be tens of thousands of dollars, which is far above our budget. If a standoff infant apnea monitor is to get to market, the cost cannot be a lot greater than that of current devices being purchased. Another concern would be the amount of processing power needed to run the algorithm and other software needed to measure breath. We are aiming for a device that can be mounted to the side of a crib, which may limit to processing power of our device.

\section{Radar Monitoring}

In 1996, researchers at the Georgia Tech Research Institute developed the Radar Vital Sign Monitor (RVSM). The technology used in the RVSM was first developed in the 1980s and under a contract from the DOD was developed to attempt to remotely measure vitals in a battle scenario of a soldier wounded or deceased [20]. The system had limitations with interference but could detect heartbeat and respiration from a distance of 100 meters.

The most recent advances in the RSVM technology were used to evaluate Olympic athlete's performance during the 1996 Olympics in Atlanta, Georgia. Specifically, they focused on archery and rifle competitors who are able to synchronize their heartbeat with their shots. The technology was designed as non-contact so as not to distract the athletes [20]. 
The transmitter radiates a signal to the athlete via an antenna. The energy that is reflected from the target is detected by the mixer diode. If there is no Doppler shirt between the signal that is transmitted and the one that is received, then there is no movement to analyze. If there is a shift between the signals, the target is moving and the signal is filtered and amplified. The filtering software can filter out the heartbeat from the respiration channel and vice versa [20]. Figure 13 shows the radar cardiogram that is observed while monitoring the athlete. The circled areas indict the accuracy and repetition that is synonymous with a heartbeat.

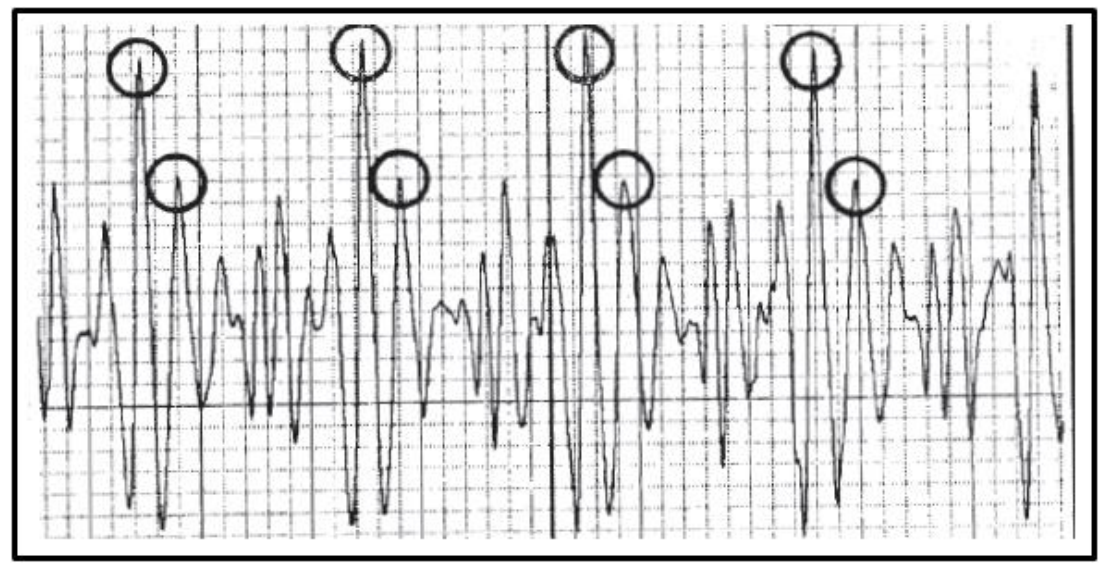

Figure 13 - Radar cardiogram of a subject using the RSVM technology[20]

This technology could have great implications in the medical world and national security. Monitoring a patient using standoff technology can be both beneficial to the patient and the doctor by easing discomfort and could eliminate the close contact between doctor and patient in sterile or quarantine situations. This technology would also allow the remote monitoring for security purposes in airports, prison, or combat situations.

The major downfall with this technology is that in its current state it is very sensitive to movement of any kind. To obtain an accurate reading the target must be very still. In emergency hospital settings patients are rarely still and the commotion in the room would interfere with the 
system. If parents are coming in to check on their infant, they would likely interfere with the radar system. With more research and development, this technology can provide substantial benefits over current biosignal monitoring.

\section{Audio Monitoring}

This section will discuss how audio has been used to monitor respiration. Monitoring the audio signatures of breath was the first technology we chose to pursue further for this thesis. Audio is a good choice for standoff technologies due to the nature of sound. To hear something does not require contact with the subject.

Audio systems have been used to diagnose different lung diseases. Auscultation is the technical term for listening to the internal system of the body [21]. Auscultation using a stethoscope is an easy and noninvasive way among physician to diagnose patient with lung diseases. This technique can be very effective and efficient for a physician with many years of experience, but the sounds one physician hears can be up for interpretation by another physician. This makes the diagnosis subjective and creates variability in the interpretation [22]. The ideal diagnostic system would be automated, so as to eliminate discrepancies between physicians.

In 2005, Taplidou et al used time-frequency (TF) analysis to study breath sounds of wheezing in asthmatic patients. Their goal was to create an automated system for wheeze detection and monitoring using spectral analysis [22]. Figure 14 represents the time frequency wheeze detection technique, from the recorded breath sound in the top plot to the wheeze detection in the bottom frequency plot. The top plot is the raw signal of the breath sound in a microphone. The spikes indicate a loud noise, in this case a breath. That signal can be put through frequency analysis software to get a plot resembling the middle one in Figure 14. The two spikes in the top plot are seen as bars in the middle plot at approximately $200 \mathrm{~Hz}$. This is the 
frequency of the breath for this individual. The bottom plot is the same as the middle but the background noise has been eliminated. The bottom plot is strictly the breath and no other background noise making the plot cleaner and easier to draw conclusions from.

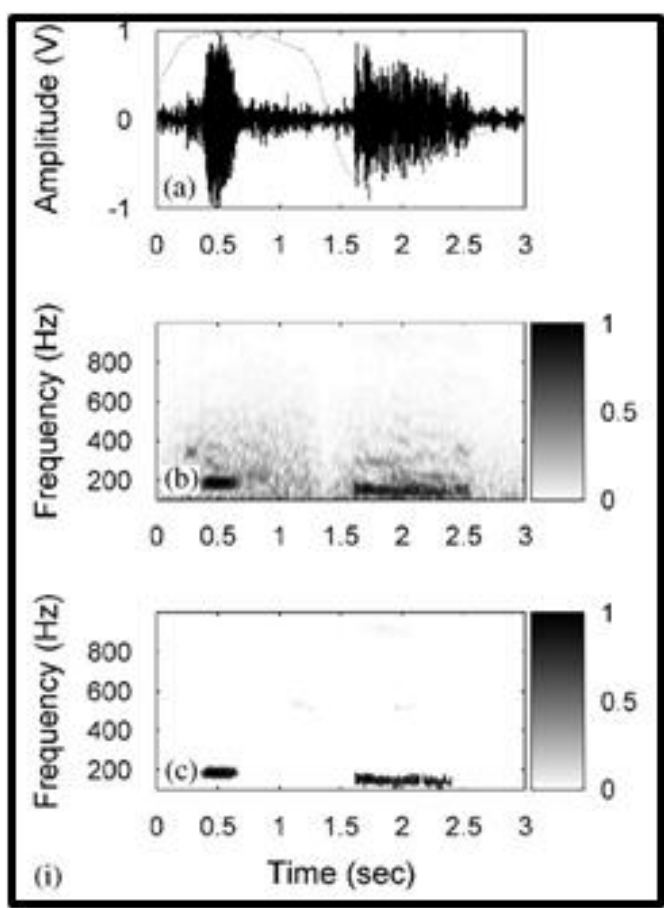

Figure 14 - Experimental results of time-frequency analysis of wheezing [22]

The results from their automated system were compared with the clinical auscultation performed by two experts. This study demonstrated that the TF-based analysis of the recorded breath sounds is an efficient and robust method for computerized wheeze detection [22]. They concluded that this wheeze detection technique could be used as an objective, fast and noninvasive tool for monitoring and evaluating wheeze activity during breathing [22].

The only difficultly with audio signatures of breath, is that they can change from person to person. There can also be a lot of variability if a person is sick and has a stuffed up nose. This makes the software behind the device more technical, as it has to account for the variability. 


\section{Carbon Dioxide Monitoring}

$\mathrm{CO}_{2}$ monitoring was the second standoff technology that was chosen for this thesis. No contact is required to measure $\mathrm{CO}_{2}$ concentrations which made it a good unit of measure for this thesis.

Carbon dioxide is regularly monitored in warehouses, offices, and in the outside atmosphere. The average atmospheric carbon dioxide level as of 2010 is $388.63 \mathrm{ppm}$ [23]. The units of $\mathrm{CO}_{2}$ are parts-per-million, or ppm, which denote relative proportions and are dimensionless. One ppm of $\mathrm{CO}_{2}$ corresponds to one molecule of $\mathrm{CO}_{2}$ in one million molecules of air. It is a measure of the amount of $\mathrm{CO}_{2}$ relative to the surrounding air. Normal indoor $\mathrm{CO}_{2}$ levels are between 400 and $2000 \mathrm{ppm}$. Level are closer to $400 \mathrm{ppm}$ outside or in an open environment. Levels can easily reach 2000 ppm in a small office during the work day. Levels above $2000 \mathrm{ppm}$ can produce an increase in drowsiness, tiredness, and headaches [24]. Monitoring $\mathrm{CO}_{2}$ levels is important in these setting to ensure dangerous levels are not reached.

Noninvasive carbon dioxide monitoring has been used to monitor mechanically ventilated infants. Normally, multiple blood samples are taken for blood gas analysis in infants with respiratory failure who are being mechanically ventilated [25]. Tobias et al studied 25 infants and toddlers under the age of 40 months who matched these criteria. They were interested in two noninvasive monitors of arterial $\mathrm{CO}_{2}$ : end-tidal $\mathrm{CO}_{2}\left(\mathrm{ET}_{\mathrm{CO} 2}\right)$ and transcutaneous $\mathrm{CO}_{2}$ (TC-CO2). $\mathrm{ET}_{\mathrm{CO} 2}$ is the partial pressure or maximal concentration of $\mathrm{CO}_{2}$ at the end of an exhaled breath, which is expressed as a percentage of $\mathrm{CO}_{2}$ or $\mathrm{mmHg}$ [26].

They showed that transcutaneous $\mathrm{CO}_{2}$ monitoring can be just as effective as blood gas analysis. Transcutaneous $\mathrm{CO}_{2}$ monitoring is not a standoff technology, but it is less invasive than 
taking blood sample. The downside the TC-CO2 is that variations in skin thickness and edema can affect the ability of the $\mathrm{CO}_{2}$ to diffuse from the capillary bed to the monitor [25]. 


\section{Apnea Monitor Design}

This section will go through the design of the standoff infant apnea monitor. It will cover the design specifications, then go into detail about the hardware and the software that was designed for both the audio system and the $\mathrm{CO}_{2}$ system. The audio system was designed by Dan White, so for extensive detail of the design of the audio system, refer to Dan's thesis. The focus of this thesis was the $\mathrm{CO}_{2}$ detection system, so most of the time will be focused on discussing the $\mathrm{CO}_{2}$ system.

\section{Design Specifications}

This project was very open-ended. We were given a small list of criteria by Raytheon and a wide window for us to pursue technologies and designs. After working in an industry for awhile, companies can get tunnel vision, and a fresh perspective can go a long way. Raytheon did not want to put limits on the project, so as to direct our ideas down one avenue. They came to us for a fresh perspective and new ideas. Keeping in mind the technologies discussed in the section titled "Standoff Technologies," we began to apply the design criteria to what we knew.

Raytheon specified that the device must have a $95 \%$ success rate. If an infant stops breathing the machine must be able to detect the apnea episode $95 \%$ of the time. There was no criterion given to false alarm rate. It would be better for the device to give multiple false alarms, but if it misses an apnea event it could mean the death of the child, which is unacceptable.

The first and biggest criterion is that the device detects breathing using a standoff approach. The great advantage to standoff technologies is that there is no need for wires being attached to the patient; everything is monitored from a distance. The exact standoff distance was not specified, as this was a proof-of-concept project. As the technology improves, we would like to maintain that $95 \%$ success rate from at least three feet and mounted to the side of the crib. 
Another major constraint was our budget. Raytheon gave us $\$ 3,500$ to use for the project. This limited the technologies we could pursue right away. Thermal imaging cameras range from $\$ 1,000$ for a low end system, to $\$ 10,000$ for the top of the line cameras. This would immediately take close to a third of our budget if we purchased a low end camera. As a proof-of-concept project we had to limit ourselves to cheaper technologies to prove they work for our application before purchasing more expensive systems.

The final housing of the device was not as important as the technology behind the infant apnea detection system. Once the technologies were designed, Scott Garrett was able to design an "infant friendly" housing for the whole system that would sit on the side of a crib. The housing would be used for the phase one testing and would not be the final device housing. The infant apnea monitor would be miniaturized before going to market and a new housing would need to be built.

\section{Standoff Technology Selection}

The decision of which standoff technologies to focus on came about from much research and discussion with Dr. Steven Barkley at Cottage Hospital. Dr. Barkley explained to us that conventional home monitors for infant apnea rely on vibrations in the infant's chest to detect breath. While vibrations may be indicative of breath they may also be indicative of struggling to breathe without actually breathing [15]. This can occur during an obstructive apnea event. We needed to find standoff technologies that could monitor true breathing, meaning actual breaths taken by the infant. The obvious question was then what is truly representative of breath. Actually measuring the inhale or exhale of the infant is the only way to truly know if the signals you are measuring are coming from a true breath. Since infants are obligate nose breathers we had to choose a technology that could focus on the air leaving their nose. 
Knowing that at least two technologies working together would give us the best result, we chose to monitor the sound of the infant's breath and monitor the $\mathrm{CO}_{2}$ concentration around in infant's nose. Dan designed the hardware setup and software for the audio system. This thesis is focused on the design of the $\mathrm{CO}_{2}$ infant apnea detection system.

\section{$\mathrm{CO}_{2}$ Design}

The following sections discuss the design of the whole $\mathrm{CO}_{2}$ detection system. The "Hardware" section will include all the components that are used to measure the $\mathrm{CO}_{2}$ concentrations. A fan system was also designed to decrease the response time of the sensor and increase the real time component of the device. The "Software" section will explain the LabView code, referred to as a block diagram, which was designed for this thesis. Each component of the block diagram will be explained in detail.

\section{$\mathrm{CO}_{2}$ Design Overview}

This section will give a general overview of the $\mathrm{CO}_{2}$ apnea detection system. In the following sections there will be great detail about the hardware and the software components that went into the detection system.

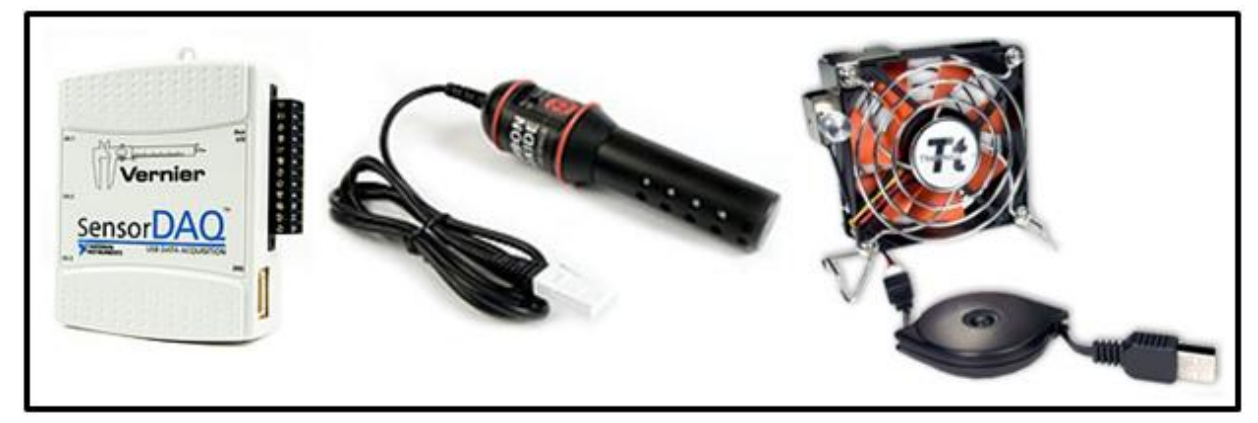

Figure 15 - $\mathrm{CO}_{2}$ Apnea Detection System Purchased Hardware

The hardware components that make up the $\mathrm{CO}_{2}$ detection system are the Vernier SensorDAQ, the Vernier $\mathrm{CO}_{2}$ sensor, the $80 \mathrm{~mm}$ USB fan with variable speed control, the white 
delrin sensor enclosure, and the safety cage to cover the fan blade. The systems that were purchased for the $\mathrm{CO}_{2}$ detection system can be seen in Figure 15. The SensorDAQ and the $\mathrm{CO}_{2}$ sensor were purchased directly from Vernier. The fan was purchased from Amazon.

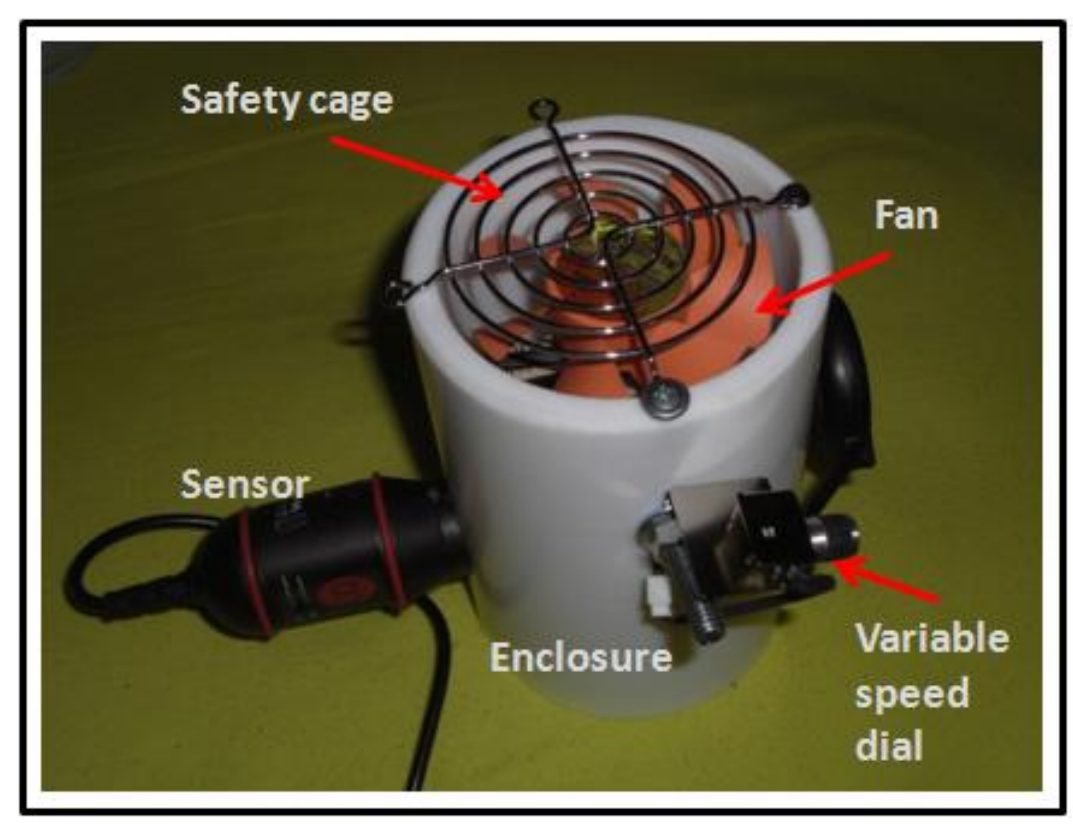

Figure 16 - $\mathrm{CO}_{2}$ Apnea Detection System

The final $\mathrm{CO}_{2}$ apnea detection system can be seen in Figure 16. A $\mathrm{CO}_{2}$ sensor was placed in a one inch hole cut into the side of the Delrin tube, so that the end of the sensor that took in the air was inside the tube. Modifications were made to the sensor that will be discussed in a later section. A hole was then drilled perpendicular to the sensor, two inches below the top of the tube. A long bolt was placed through the tube in the hole. The fan was then mounted to the bolt using an adhesive backing and wire ties. The fan was oriented so that the air was pulled into the tube and pushed over the sensor. The wires from the fan were threaded out a small hole that was drilled opposite the sensor. The fan has a variable speed dial that was mounted to the portion of the bolt that protruded from the delrin tube. The fan also had a retractable cord that was mounted with Velcro on the side opposite the sensor. 
That system is connected to the Netbook through the Vernier SensorDAQ. The $\mathrm{CO}_{2}$ sensor attaches to one of the three ports on the left of the SensorDAQ and the SensorDAQ attached to the laptop using USB. Both the fan and the $\mathrm{CO}_{2}$ sensor connect to the computer via USB, reducing the number of devices that need an external power source, such as a wall outlet. The final $\mathrm{CO}_{2}$ detect system seen in Figure 16 can be mounted to the side of the crib or mounted to the housing system that was designed for testing, which will be discussed in a future section.

\section{$\mathrm{CO}_{2}$ Detection System Materials}

The following sections will give a detailed description of the hardware components that make up the $\mathrm{CO}_{2}$ apnea detection system. This includes the components can be seen in Figure 15 and Figure 16.

To detect carbon dioxide, a sensor is needed to collect the gas, a fan is needed to pull the air over the sensor more rapidly, a data acquisition system (DAQ) is needed to link the sensor to LabView, and a display is needed to monitor and view the carbon dioxide levels and apnea events. All of these components will be discussed at length in the following sections.

\section{Vernier $\mathrm{CO}_{2}$ Sensor}

A Vernier $\mathrm{CO}_{2}$ sensor was chosen for its compatibility with National Instruments products, such as LabView. Both companies design products for classroom settings so the interfaces are very user friendly. It was also important that the sensor be able to read in real time carbon dioxide concentrations. In an infant apnea event the detection system has 20 seconds to detect the event and alert the parents. A large portion of the carbon dioxide sensors of the market are used for monitoring large spaces, such as a warehouse or office spaces, so the response time and accuracy are not critical. They monitor environments on the order of \pm 10 to $20 \mathrm{ppm}$ and take 
a reading every 30 minutes to an hour [24]. Our detection system needed a sensor that could detect the changes on the order of $1 \mathrm{ppm}$, take a reading in real time, and could detect the high levels of carbon dioxide that are expelled during breathing.

\section{How the $\mathrm{CO}_{2}$ Sensor Works}

The Vernier $\mathrm{CO}_{2}$ sensor measures gaseous carbon dioxide levels in the range of 0 to $10,000 \mathrm{ppm}$ in the low setting, and 0 to $100,000 \mathrm{ppm}$ in the high setting. The high setting is used for monitoring events such as respiration of animals and the low setting is used to monitor levels around plants in a terrarium. It monitors the amount of infrared radiation absorbed by carbon dioxide molecules. It uses an LED as the source to generate infrared radiation. The LED is located on one end of the sensor's shaft, while an infrared sensor is located on the other end. The infrared sensor measures how much radiation gets through the air without being absorbed by the carbon dioxide molecules. The detector measures radiation in the narrow band centered at 4260 $\mathrm{nm}$. The greater the concentration of the absorbing gas in the sampling tube, the less radiation will make it from the source, through the sensor tube, to the infrared detector which experiences a temperature increase. That temperature increase produces a voltage that is amplified and read by the Vernier interface (SensorDAQ) [27]. $\mathrm{CO}_{2}$ gets to the sensor by diffusion, which is a passive and slow reaction. This allows for much of the $\mathrm{CO}_{2}$ from a breath to diffuse out into the room before it gets to the sensor, which would then make $\mathrm{CO}_{2}$ monitoring an inaccurate representation of infant breath.

\section{Sensor Modifications}

The sensor tube has 20 holes to allow the gas to diffuse in and out of the tube. Diffusion is a slow process, so sudden changes in $\mathrm{CO}_{2}$ levels may take several seconds to be recognized by 
the sensor. For this thesis, small changes $( \pm 20 \mathrm{ppm})$ need to be recognized in less than 20 seconds or the device is useless at detecting infant apnea. If the device cannot detect small changes, the infant might have been not breathing for several minutes before the diffused air reaches the sensor and in interpreted by the software.

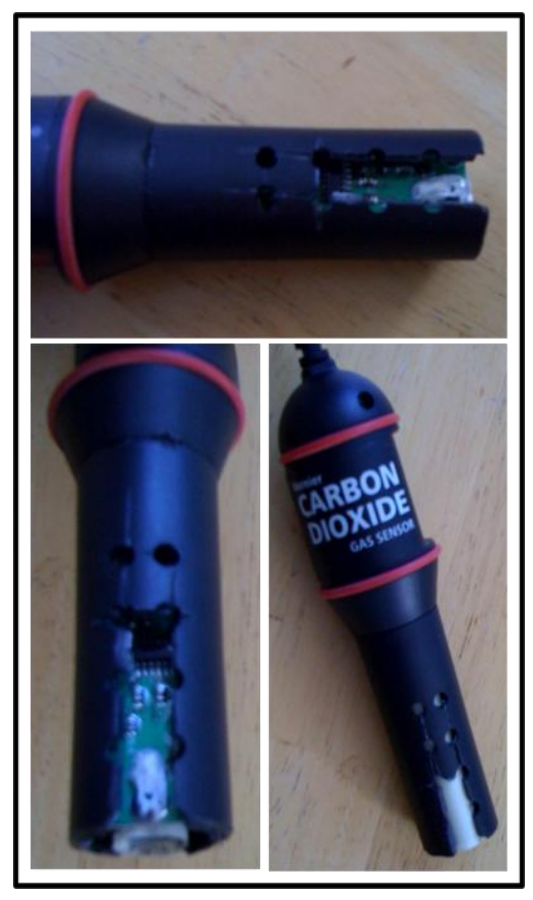

Figure 17 - Modifications made to the $\mathrm{CO}_{2}$ sensor

Modifications were made to the sensor to attempt to increase the affinity for the $\mathrm{CO}_{2}$ molecules to enter the sensor tube. These modifications can be seen in Figure 17. A portion of the sensor casing was removed to allow the air to diffuse to the sensor tube more rapidly. The air was allowed direct contact with the sensor tube instead of having to travel through the holes in the plastic casing. This would help reduce the time between the carbon dioxide leaving the infants mouth and the sensor detecting the fluctuations.

\section{Fan Integration}

Infants have a very small lung volume, so it is essential that as much of the exhaled air is captured as quickly as possible. To overcome the slow process of diffusion and saturation, a 
small fan was incorporated. Two different sized fans were tested to obtain the best results. Both fans were exactly the same except for size. A 40 x $40 \mathrm{~mm}$ and an 80 x $80 \mathrm{~mm}$ USB computer fan were tested. Results of the tests can be found in the "Fan Results" section.

The fan was used to pull the air from around the infant's nostrils to the sensor to obtain real time data before the volume of air is dissipated throughout the room. The USB allowed the fan to be powered by computer and eliminated the need for a power outlet. The fan was variable speed, so the best setting could be found that was compatible with the audio system. The fan does produce sound, so there is a possibility for the fan noise to drown out the noise of the infant. Finding the proper speed was crucial to ensure this problem did not arise.

Experiments were conducted both pulling and pushing the air across the sensor, seen in Figure 18. Diagram A, on the left side, demonstrates the pulling scenario. The air enters the delrin tube from the left and is pulled over the sensor by the fan and out the right side. Diagram B shows the fan pushing the air over the sensor and out the right side. The results from these tests can be found in the "Fan Results."

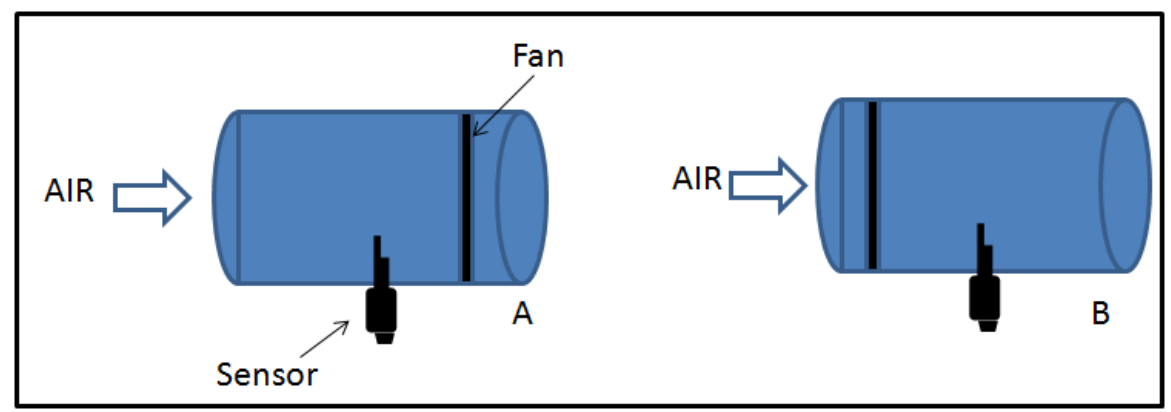

Figure 18 - Fan Configurations

\section{Detection System Enclosure}

An enclosure needed to be design to house all the components together. A delrin tube was used with dimensions: 4 inch diameter, 6 inches tall, and $1 / 2$ inch thick. This tube was used 
because it was leftover from a previous project and fit the dimensions needed to house the fan. Figure 19 shows the enclosure that was designed from different angles. Figure 19a shows the fan mounted in the enclosure with the safety cage on top. The hole for the sensor can also be seen in this figure. Figure 19b shows the variable speed dial mounted to the bolt and the retractable fan cord mounted to the side if the enclosure, using Velcro. A bottom view showing how the sensor fits into the enclosure is seen in Figure 19c. Figure 19d shows another angle of the final $\mathrm{CO}_{2}$ detection hardware system.

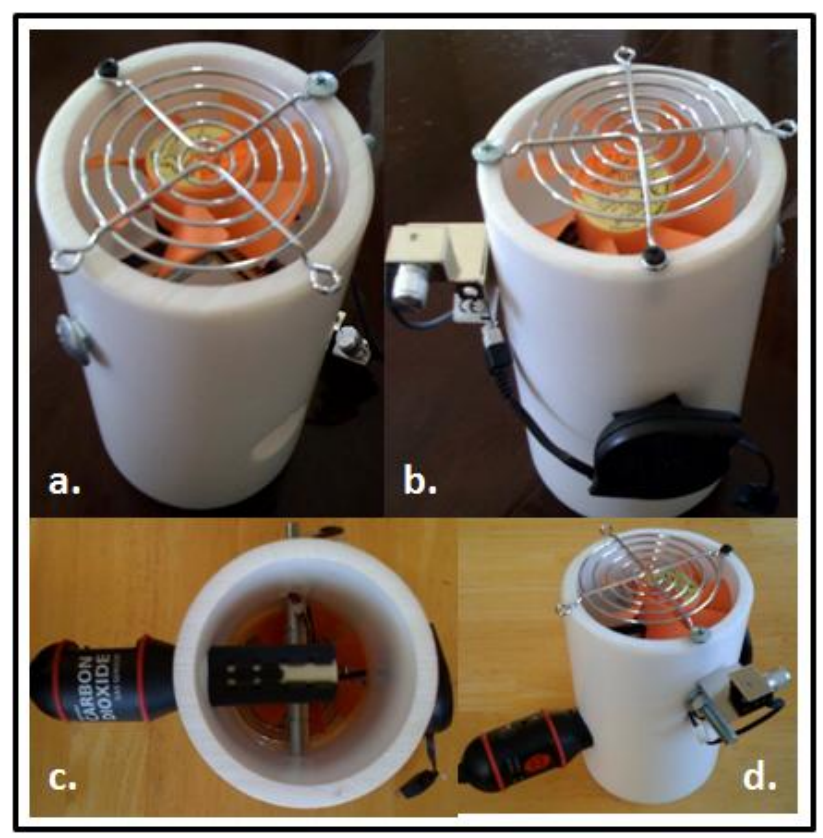

Figure 19 - $\mathrm{CO}_{2}$ Detection System Enclosure

\section{$\mathrm{CO}_{2}$ Apnea Detection Software}

National Instrument's LabView was an obvious choice for us to design the software for the standoff infant apnea monitor. It allows for multiple systems to interface with each other without interference. LabView allowed Dan and me to develop the software to the individual audio and $\mathrm{CO}_{2}$ monitoring systems separately. Intermittently we could combine them to run the system as a whole as we worked towards a final LabView system. It is readily available in the 
Biomedical Engineering labs on Cal Poly's campus. National Instruments hardware and software packages can be thousands of dollars, but as graduate students we have access to this equipment at no additional charge to us. The software written could be written into an executable file that could be run on any computer, specifically the Netbook used in this project. This means that LabView does not have to be loaded onto the computer that runs our apnea detection program. The file allows the user to run the program, but not alter any of the background code.

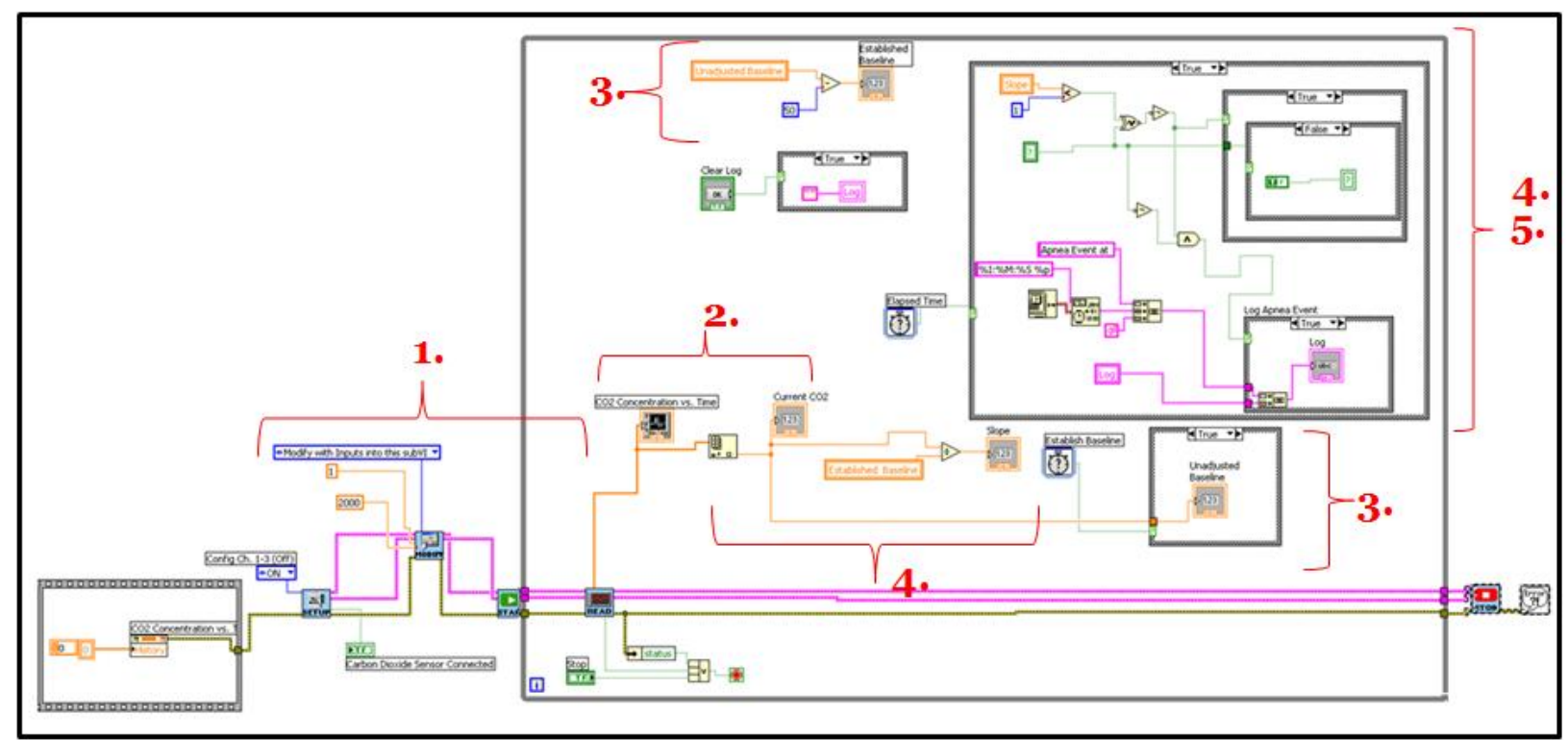

Figure 20 - Block diagram of $\mathrm{CO}_{2}$ detection system

LabView allows software to be developed using pictorial representations instead of words as code. Figure 20 shows a screenshot of the block diagram which contains the code for the carbon dioxide monitoring system. The number sections (1-5) are different sections of the code that are all tied together for the overall system. The large gray rectangles surrounding different section within the block diagram allow the code inside to be looped until certain conditions are met, similar to a "For Loop" or a "While Loop" in other programming languages. The following is a detailed description of what each number section in Figure 20 does. 
1. The smaller gray rectangle on the far left is what clears all the data every time a new test is run. Once the values are set back to the initial settings, the data can be read in from the $\mathrm{CO}_{2}$ sensor. This section reads in the raw carbon dioxide values from the sensor through the SensorDAQ. The SensorDAQ comes with a couple blocks that can be modified for specific applications. The first few blocks specify which channel the sensor is plugged into the SensorDAQ. In the user interface a green light will illuminate when the $\mathrm{CO}_{2}$ sensor is attached. The "Modify" block allows the user to change the amount of sample that are taken and the rate at which they are sampled at by the sensor. "Start" allows the user to click to begin reading in $\mathrm{CO}_{2}$ concentrations. This takes place in section 2, where the carbon dioxide levels (ppm) are plotted verses time (sec), and the current $\mathrm{CO}_{2}$ value is visible as the program runs.

2. Once the data is read in it needs to be manipulated and displayed. The raw $\mathrm{CO}_{2}$ concentrations are plotted on a graph of $\mathrm{CO}_{2}$ concentration verses time, in seconds. As the values are plotted, a digital reading is shown directly above the graph. Now the software needs to take those values of $\mathrm{CO}_{2}$ and determine whether an apnea event is occurring or not. This takes places between sections 3 and 4 .

3. First step is to establish a baseline $\mathrm{CO}_{2}$ value. When a test is initiated, the first 30 seconds of data are used to establish a baseline $\mathrm{CO}_{2}$ level with the infant in the crib. At 30 seconds, the current $\mathrm{CO}_{2}$ value is recorded as an "Unadjusted Baseline." 50 ppm are then subtracted from that "Unadjusted Baseline" to get an "Adjusted Baseline." This technique of finding a baseline was seen in a published journal article [12]. Now we can compare all incoming $\mathrm{CO}_{2}$ values to the baseline to determine if an apnea event is taking place. 
4. Section 4 is where the software determines if an apnea event is occurring or not. The incoming $\mathrm{CO}_{2}$ concentrations are compared to the "Established Baseline." If the slope of the ratio of the two is less than one, a timer starts. If the slope remains less than one for 20 seconds, an apnea event is detected and the illuminated green bar turns to red to indicate apnea.

5. Once an apnea event is detected, it is logged. This is done in section 5 in Figure 20. It is recorded as "Apnea Event Occurred at:" plus the time it was detected. Each event is only logged once, so if the apnea continues to occur, multiple events will not be recorded. This is an important feature for initial testing of the system and reviewing apnea events throughout the course of a night.

These five sections are what make up the LabView software for detecting infant apnea. Later there will be software modifications that will be suggested for future generations of the standoff infant apnea monitor.

\section{Audio Design}

The audio system was designed by Dan White. This section will be a broad overview of the components that went into the audio system. Breathing produces sound at varying frequencies and volumes, which can then be analyzed to determine if breathing is taking place. For further explanation of the audio system, read Dan White's thesis.

The hardware for the system includes two microphones, a dual USB audio interface, and preamplifier. Infant's breath sounds are quiet enough that a passing car, ventilation system, or washing machine can drown out the breath sounds, not allowing the microphone to detect the breath. To overcome this, one of the microphones is unidirectional and one is omni-directional, seen in Figure 21a, b, respectively. The directional microphone is pointed at the infant and the 
omni-directional is pointed away, toward the rest of the room. The omni-directional microphone picks up all sounds in and around the infant's crib. The audio picked by the omni-directional microphone is subtracted from the directional microphone to ensure the audio that is processed by the software is solely the infant's breath. The microphones and the sound card in the computer create noise that is separated using the preamplifier, seen in Figure 21c. It also allows for the two microphones to be integrated into the computer as two separate channels.

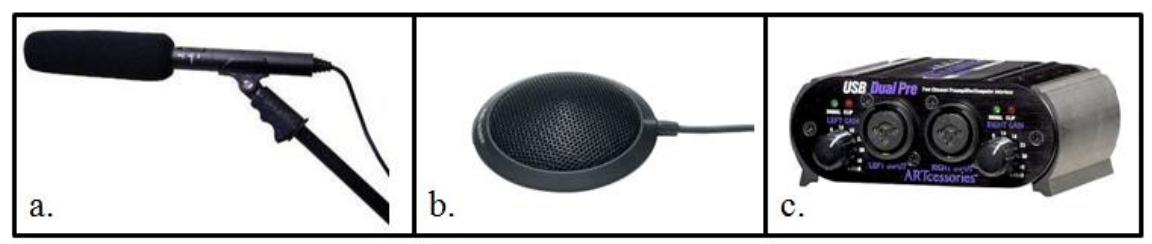

Figure 21 - Audio System Hardware

The software for the audio was designed in National Instruments LabView. The sound from each microphone is passed through a band pass filter and an instantaneous measurement is displayed and set as a baseline value. As each new instantaneous measurement is recorded it is divided by the baseline and compared to a threshold value of 1.1. The threshold value was determined previously by running tests with values both above and below 1.1 . If the threshold is exceeded, a breath is counted. If the measurement is below threshold, a clock starts counting "time since last breath." Once "time since last breath" reaches "time until apnea," which can be specified by the user, the apnea alarm is trigger. For example, if breath has not been detected from the infant for approximately 15 seconds, an apnea event is detected, and the alarm goes off, alerting the parents the infant may be in distress. Not only are the parents alerted, but the apnea event is logged for future reference. There has also been a feature built into the audio software to $\log$ "near apnea events." This means that if the "time since last breath" equals a fraction of the "time until apnea," meaning that breath has not been detected in 10 second, but not long enough 
to record an apnea event, it can be logged. This can also have future benefits for a physician to diagnoses.

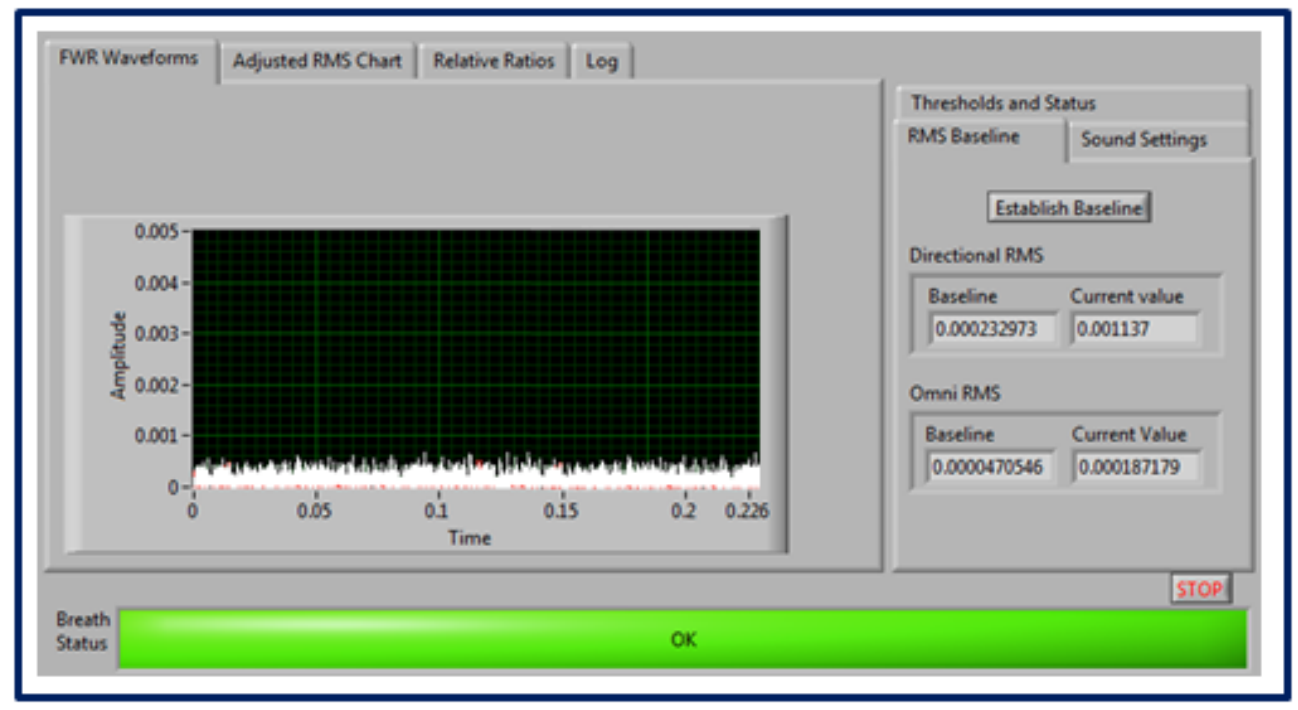

Figure 22 - Audio Detection System Front Panel

Figure 22 shows the LabView front panel for the audio detection system. Each tab in the upper left displays either a graph of the audio analysis being done or a log of near apnea or apnea events. The tabs on the right display some of the real time results from the audio analysis, such as the threshold and the baseline that are used to determine if an apnea event is occurring. The large green icon is the apnea indicator. If it is green and reads "OK," then the infant is breathing normally and if it turns red and reads "APNEA," then no breath is detected. The code behind this user interface is presented in Dan White's thesis and should be referred to for more information.

\section{Integration of Audio and $\mathrm{CO}_{2}$}

This section will discuss the process of combining the audio system with the $\mathrm{CO}_{2}$ system. Both systems, audio and $\mathrm{CO}_{2}$, were developed separately throughout the course of this thesis. Periodically they would be combined to test for problems that each system might cause for the other. The hardware could be kept separately, but the LabView software had to be integrated. 
National Instruments makes the combination of different LabView codes very straightforward. The audio block diagram was used as the primary template and the $\mathrm{CO}_{2}$ block diagram was then imported into the audio system. The entire $\mathrm{CO}_{2}$ block diagram code was selected and "dragged" over into the audio block diagram. Some blocks were moved around so as to make the front panel easy to use. A visual representation of the front panel and the block diagram can be found in Figure 23 and 24, respectively.

Figure 23 shows the front panel of the audio portion on top and the $\mathrm{CO}_{2}$ portion on the bottom. The portion that was added when integrating the systems was the dark green bar circled in red between the two systems. In the current view, both detection systems are light green meaning and the infant is breathing normally. If both detection systems indicators turned red the dark green bar would also turn red indicating an apnea event. 


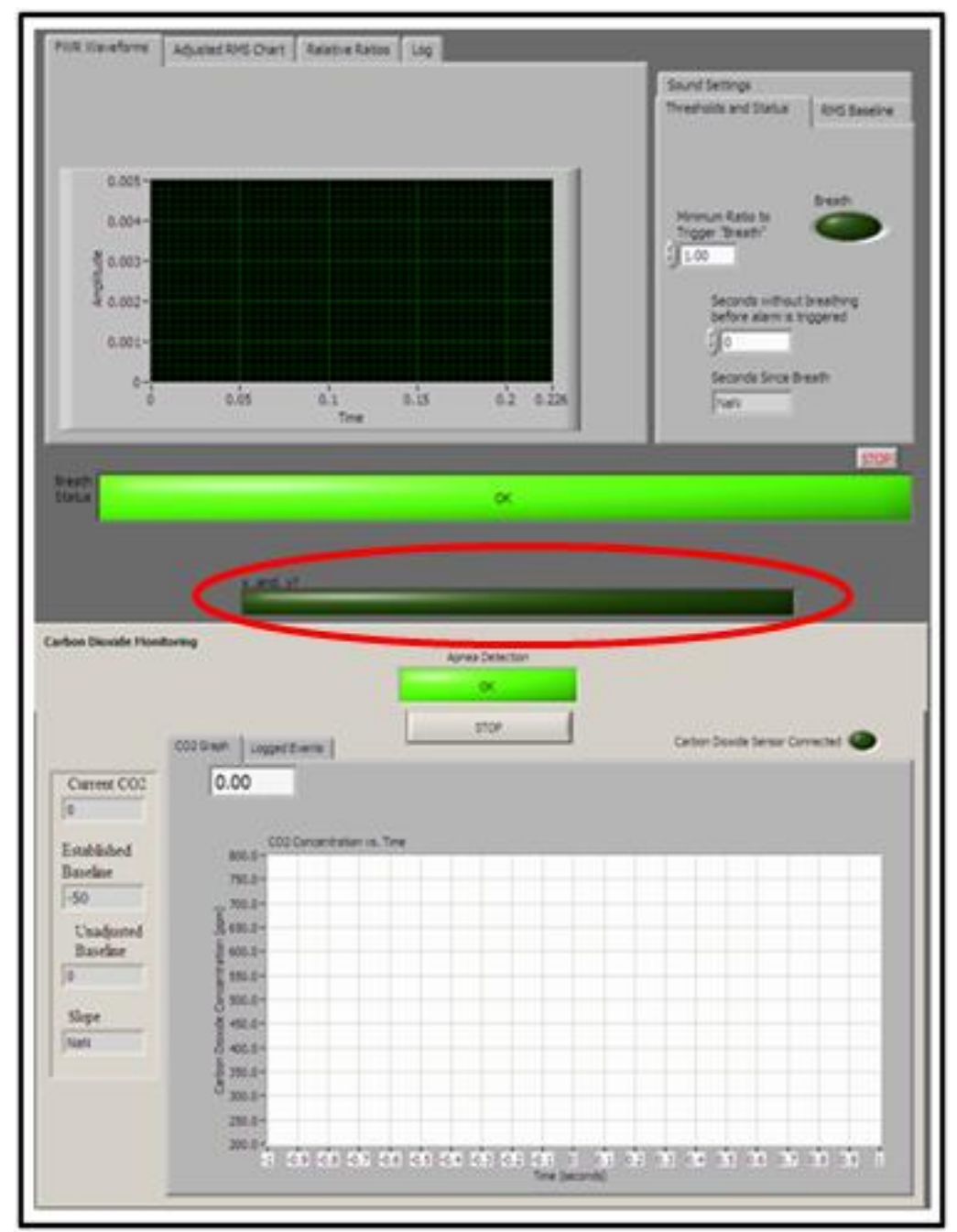

Figure 23 - Front Panel of Infant Apnea Monitoring System

Figure 24 shows the block diagram of the integrated system. The top portion is the block diagram from the $\mathrm{CO}_{2}$ code and the bottom portion in the code for the audio portion. The section of code circled in red represents the code that was added to tie the two detection systems together. If the $\mathrm{CO}_{2}$ system detects apnea at the same time the sound system does the alarm will go off indicating an apnea event. A "Stop" icon was added to be able to start and stop the whole system simultaneously. This is the only code that was needed to integrate the systems. The individual system codes still run independently as was described in previous sections. 


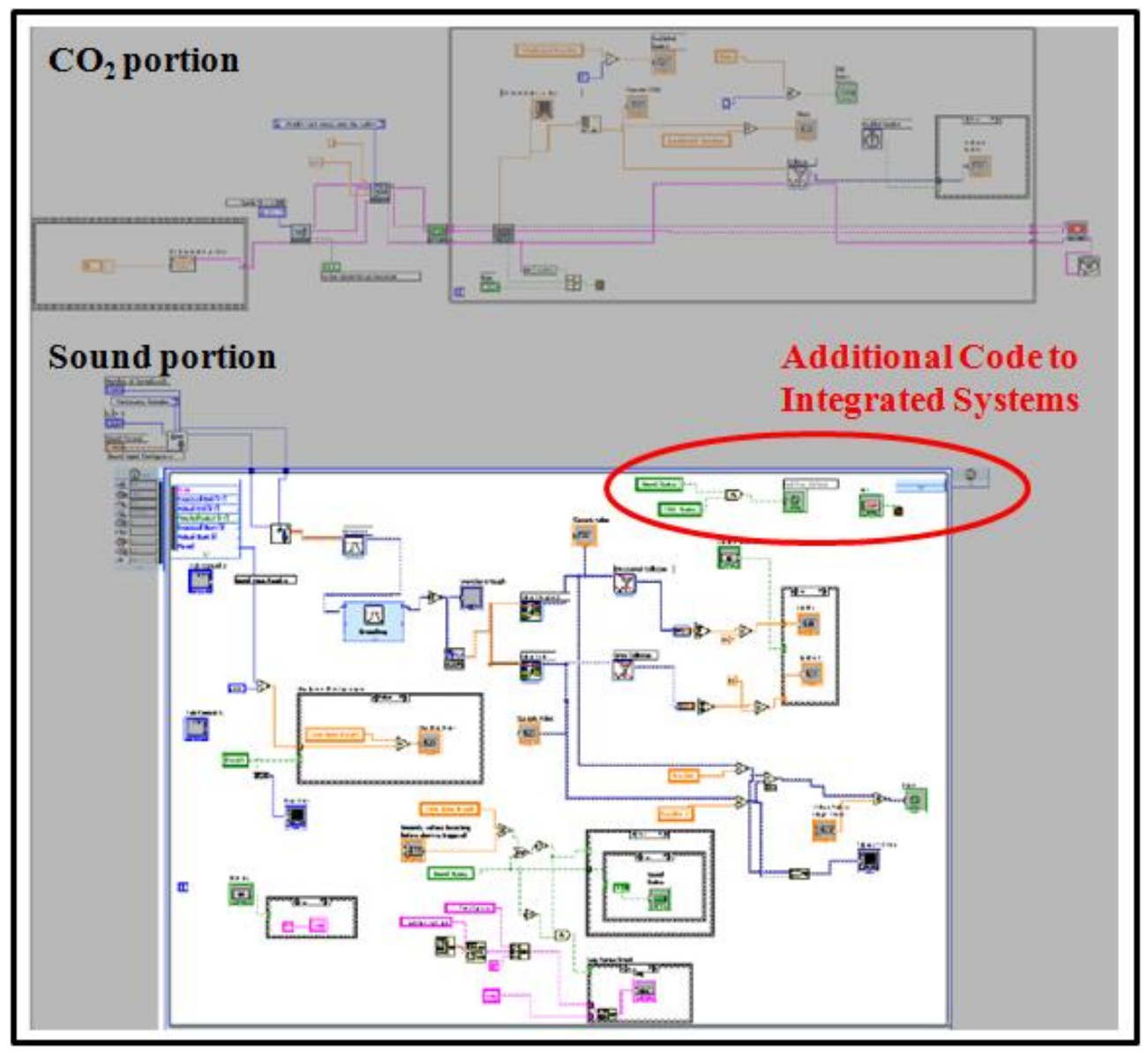

Figure 24 - LabView block diagram of integrated code

\section{Monitor Crib Side Module}

This section will briefly discuss the crib side module that was designed for the proof-ofconcept standoff infant apnea monitor. This enclosure would not be used for the final product, as the monitor would be miniaturized before going to market, but it would be used during preliminary testing of the device in a home setting.

Figure 25 show three different views of the module that Scott Garrett designed for the monitor. In Figure 25a the Netbook that runs the software sits on the gray shelf that is mounted on the outside of the rungs of the crib. Figure 25b shows how the shelf mounts to the rungs of the crib. A gray plate sits on the inside of the rungs and is bolted to the shelf on the outside of the 
crib. To ensure the enclosure does not slide down or come loose, it is also tied to the top of the crib with rope. Figure $25 \mathrm{c}$ shows an infant friendly monkey covering for the enclosure. The monkey arms cover the rope that secures the enclosure to the top of the crib. For safety, all the edges were rounded and felt was used to cover the enclosure.

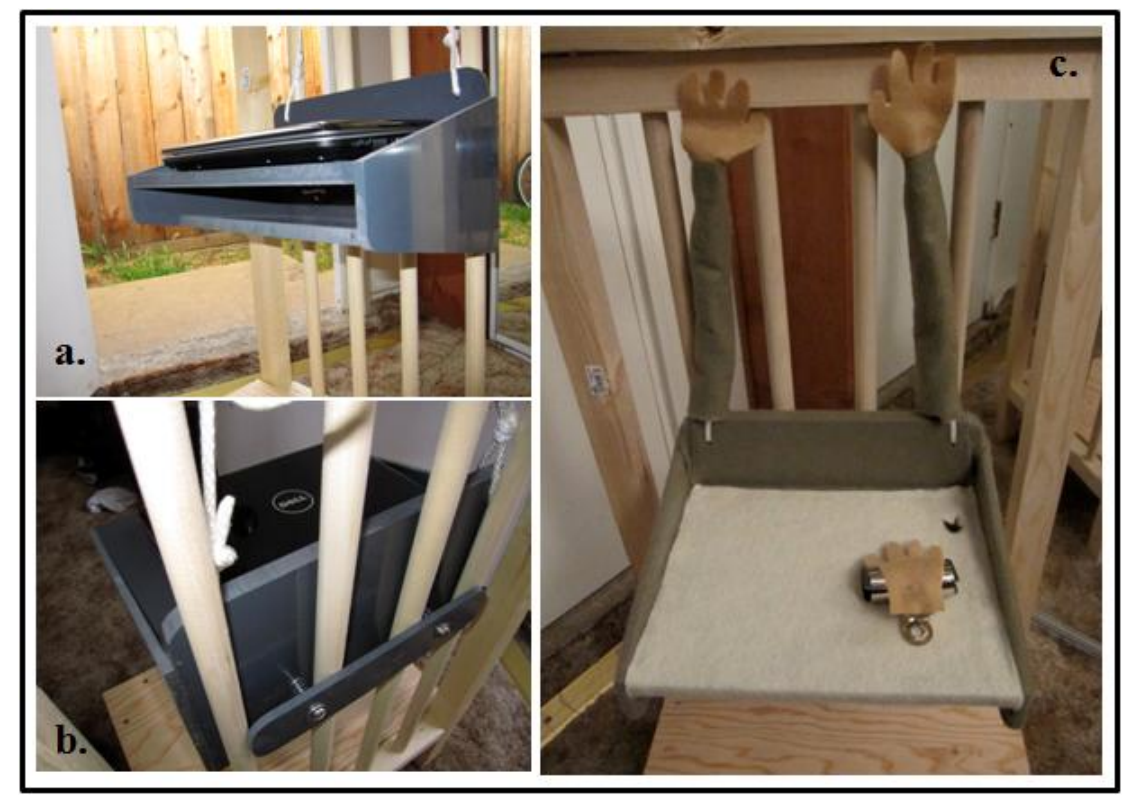

Figure 25 - Standoff Infant Apnea Crib side Module

Figure 26 shows the underside of the crib side module seen in Figure 25. This is where the SensorDAQ, the USB audio Amp, and the omni-directional microphone are mounted. There is also a port for all the USB cords to come up through to connect to the laptop that sits on the opposite side. This allows all the hardware to be out of the way during testing. 


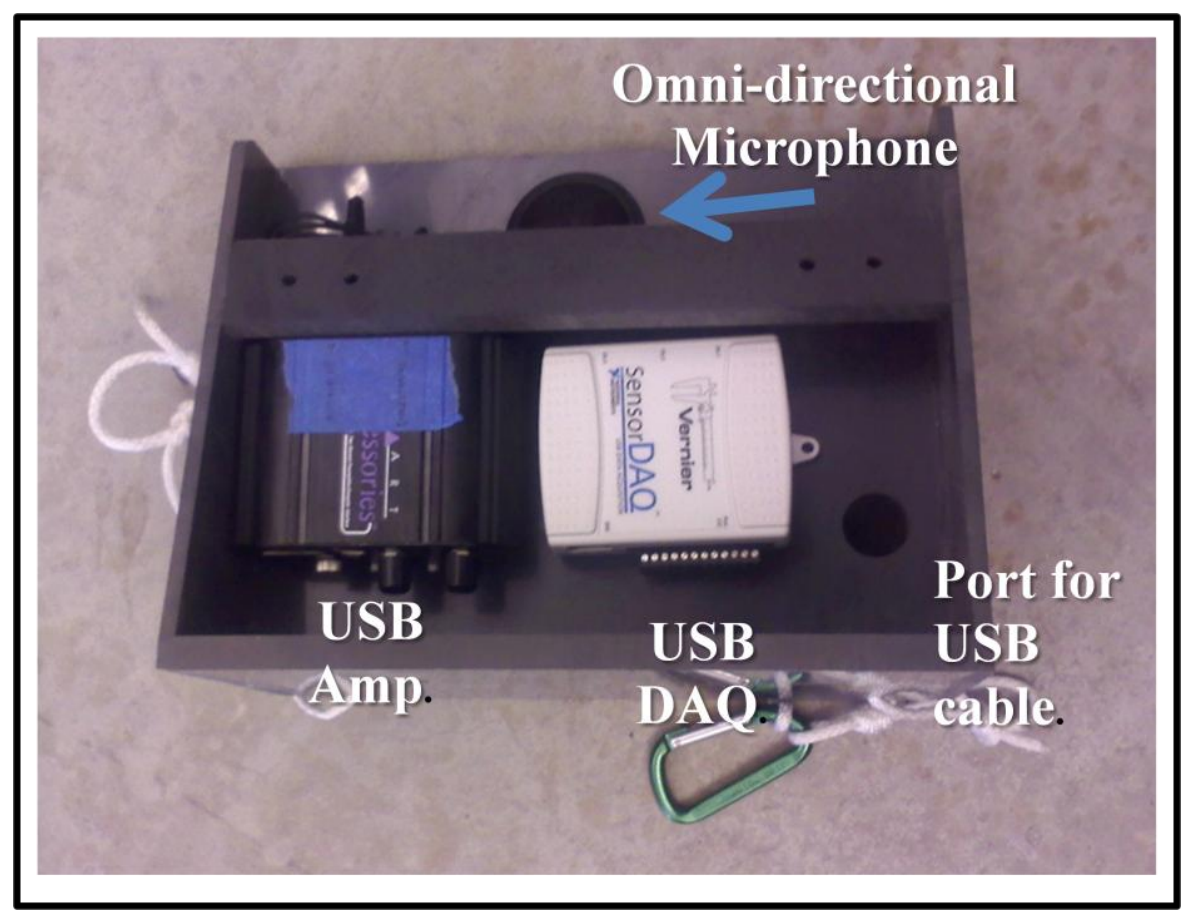

Figure 26 - Underside of Crib Side Module 


\section{Standoff Apnea Monitor Testing and Results}

Once the standoff infant apnea monitor was designed, it needed to be tested. The independent detection systems were tested separately, as well as together. For the purpose of this thesis, majority of the testing was focused on the $\mathrm{CO}_{2}$ apnea detection system. Initial testing was conducted on adults, in which the hypothesis was that the $\mathrm{CO}_{2}$ apnea detection system could detect adult breath and detect simulated apnea events from a standoff position. The overall hypothesis being tested was that the $\mathrm{CO}_{2}$ detection system can detect infant breath from a standoff position.

This section will discuss the testing that was done on both adults and infants for the $\mathrm{CO}_{2}$ detection system. The adult testing will include the sensor validation, fan testing, enclosure testing, and overnight testing. Next, the infant testing will be discussed. The infant testing was done in the Neonatal Intensive Care Unit (NICU) at Cottage Hospital in Goleta, CA. After each section the results of the testing will be displayed. They will only be presented here and not interpreted. They will be discuss and interpreted in the section titled "Testing Conclusions and Discussion."

\section{Adult testing}

All of the adult testing, with the exception of the overnight testing, was done with the adult breathing through their nose, to best simulate infant breathing. The breathing during overnight testing could not be controlled, as the adult was asleep. Conclusions from the overnight testing could only be interpreted for adult apnea. These interpretations will be discussed on a later section. 
Initial measurements were taken using an adult subject to get an idea of the response time of the $\mathrm{CO}_{2}$ sensor. Knowing that the detection of infant apnea is very time sensitive, testing was done to ensure the fastest response time from the sensor. Additional adult testing was done over several nights to look for interferences between the systems if they were run for long periods of time and looking at false alarms. The overnight testing results are displayed after the NICU testing results, as that was the order the testing was done. The following sections display the results from the adult testing that was done to ensure the sensor could detect an apnea event with enough time to alert the parents. That time is referred to as response time. The results will then be discussed in the next section titled "Testing Conclusions and Discussion."

\section{$\mathrm{CO}_{2}$ Sensor Validation Testing}

To ensure the $\mathrm{CO}_{2}$ sensor was properly calibrated, validation testing was performed. A published $\mathrm{CO}_{2}$ value of $388.63 \mathrm{ppm}$ was researched and used to compare readings from the $\mathrm{CO}_{2}$ sensor that was purchased [24]. A series of fifteen readings were taken outdoors using the Vernier $\mathrm{CO}_{2}$ sensor. For each reading the sensor was run for five minutes and an average $\mathrm{CO}_{2}$ value during those five minutes was recorded in Table 4. The developed LabView software is capable of displaying the minimum, maximum, and average $\mathrm{CO}_{2}$ concentration obtained from each test. The recorded values were then compared to the researched value of $388.63 \mathrm{ppm}$. Statistical analysis was done using Minitab to observe if the recorded values were statistically similar to the published value. The results are presented in the following section and will be discussed in the section titled "Testing Conclusions and Discussion." 


\section{$\mathrm{CO}_{2}$ Sensor Validation Results}

The average $\mathrm{CO}_{2}$ concentration obtained during each test can be found in Table 4. The results from each test were then averaged together to get an overall average. The average measured $\mathrm{CO}_{2}$ reading was $390.10 \mathrm{ppm}$ with a standard deviation of $2.89 \mathrm{ppm}$. The results did appear to fluctuate from test to test but appeared to stay relatively close to the published value of 388.63 ppm.

Table 4 - Average Measured $\mathrm{CO}_{2}$ Values

\begin{tabular}{|cc|}
\hline $\begin{array}{c}\text { Test } \\
\text { Number }\end{array}$ & $\begin{array}{c}\text { Measured } \\
\text { CO }_{\mathbf{2}} \text { (ppm) }\end{array}$ \\
\hline 1 & 389.2 \\
2 & 388.85 \\
3 & 390.6 \\
4 & 385.32 \\
5 & 393.69 \\
6 & 391.98 \\
7 & 390.21 \\
8 & 394.43 \\
9 & 387.67 \\
10 & 390.54 \\
11 & 388.2 \\
12 & 391.33 \\
13 & 395.29 \\
14 & 386.45 \\
15 & 387.74 \\
\hline
\end{tabular}

The measured values were compared to the published value of $388.63 \mathrm{ppm}$ to look for significant differences or similarities. Using Minitab, an Anderson-Darling Normality Test was performed. A p-value of 0.907 was obtained, meaning there was no statistical difference between the measured values and the published value. A 95\% confidence interval was found for the mean of the measured $\mathrm{CO}_{2}$ values. The $95 \%$ confidence interval was $(388.49,391.79)$. The boxplot in 
Plot 1 shows the differences between the measured $\mathrm{CO}_{2}$ values and the published $\mathrm{CO}_{2}$ value. The published, or hypothesized, value is the red dot and falls in the $95 \%$ confidence interval.

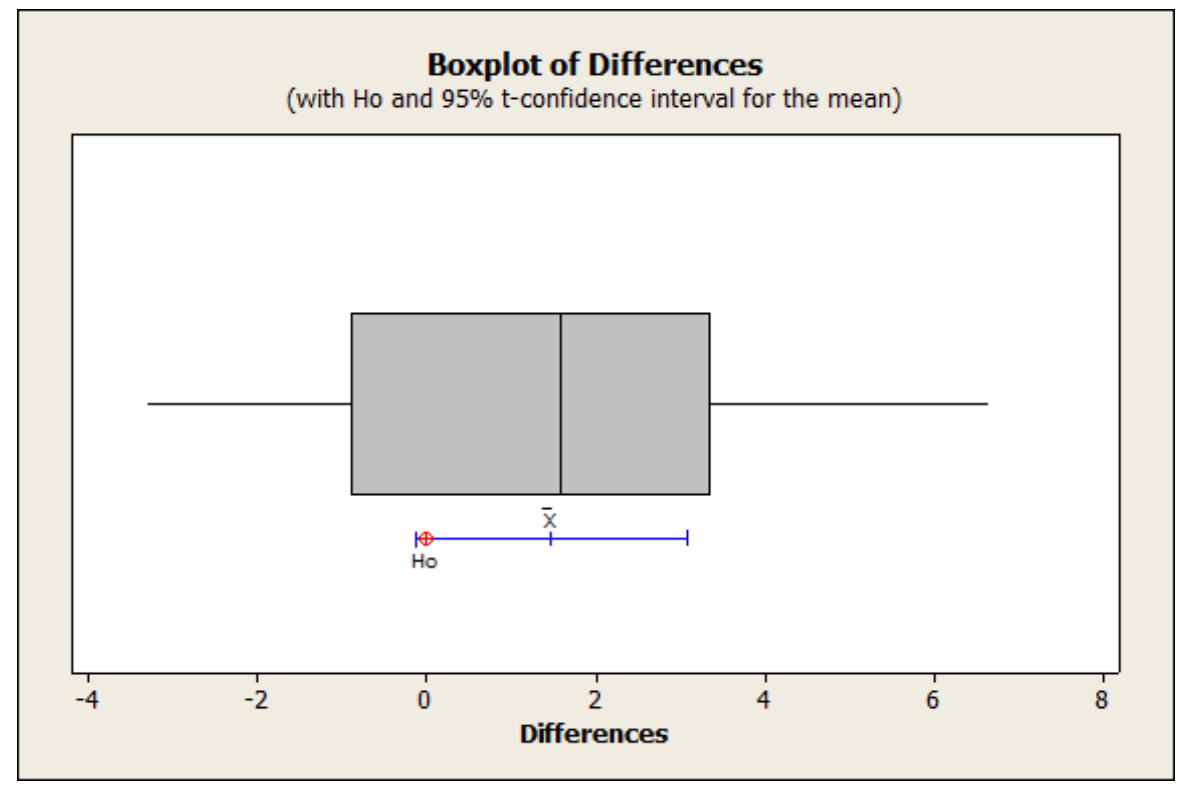

Plot 1 - Boxplot of Differences between measured and hypothesized $\mathrm{CO}_{2}$

These results show that the concentrations the $\mathrm{CO}_{2}$ sensor was reading are statistically similar to the published value, validating the sensor. With the sensor validated, it could be used for further testing. The validation results and their implications will be discussed in the "Validation Conclusions" section.

\section{Solo $\mathrm{CO}_{2}$ Sensor Testing}

After the sensor was validated, testing was done to see how fast the sensor could detect a simulated apnea event without any modifications to the sensor. Before testing, a baseline $\mathrm{CO}_{2}$ concentration of the room was recorded. The sensor was held one foot from the adult test subject's nose, with their hand resting on a rigid table to keep the sensor steady. The subject breathed into the sensor through their nose only, for one minute. They were then instructed to turn around and breathe away from the sensor, to simulate no breath over the sensor. The time it 
took the sensor to detect the simulated apnea event was recorded. The test was run 10 times to get a range of data. Time was allotted between tests to ensure all the $\mathrm{CO}_{2}$ concentration was back to the baseline.

\section{$\mathrm{CO}_{2}$ Sensor Results}

Before testing began, a baseline $\mathrm{CO}_{2}$ concentration of $394.2 \mathrm{ppm}$ was recorded. The results from the 10 tests are displayed in Table 5. The average response time was 29.43 seconds. The implications of these response times will be discussed in a later section.

\section{Table 5 - Response Time of $\mathrm{CO}_{2}$ Sensor}

\begin{tabular}{|cc|}
\hline $\begin{array}{c}\text { Test } \\
\text { Number }\end{array}$ & Response Time (sec) \\
\hline 1 & 30.14 \\
2 & 29.6 \\
3 & 27.42 \\
4 & 29.79 \\
5 & 29.62 \\
6 & 30.07 \\
7 & 28.94 \\
8 & 28.81 \\
9 & 29.77 \\
10 & 30.14 \\
\hline
\end{tabular}

\section{Fan Testing}

It is important to minimize the time it takes for the sensor to recognize a lack of breath and interpret that as an apnea event. To ensure the fastest time possible, modifications were made to the sensor and additional testing was performed.

Testing was done to determine if a fan would enhance the real time reading of the $\mathrm{CO}_{2}$ sensor. Once we obtained data for a single fan, testing was done to evaluate the use of multiple fans to further decrease response time. This section will discuss the testing that was done with 
both a single fan and multiple fans, with adult test subjects. It was known that the test subject did not have any form of apnea. This required them to simulate apnea by hold their breath for 15 seconds at a time. If at any point the test subject felt uncomfortable holding their breath the test was aborted to ensure the safety of the test subjects.

The first tests that were conducted were done with both the 80mm USB fan and the 40 mm USB fan. This consisted of the fan and the $\mathrm{CO}_{2}$ sensor. The sensor was mounted to a table and the subject was sitting in a chair. The distance between the subject's nose and the sensor was fixed at two feet.

Each test was run for one minute. The subject was asked to hold their breath after 30 seconds of recording. The time it took for the $\mathrm{CO}_{2}$ detection system to recognize the simulated apnea event was recorded as response time. If one fan performed better at one distance it was assumed that it would also perform better at other distances. The fan was placed approximately 3 inches from the sensor so that it would pull the air from the subject's nose and push the air over the sensor. A visual representation can be seen in Figure 27. These tests were also done with the $40 \mathrm{~mm}$ fan and without a fan. Another set of tests was conducted with the fan 3 inches behind the sensor. The data was compared using statistical analysis to draw conclusions about the benefits of adding the fan. The results of these tests can be found in following sections and those results will be discussed in the section titled "Fan Conclusions." 


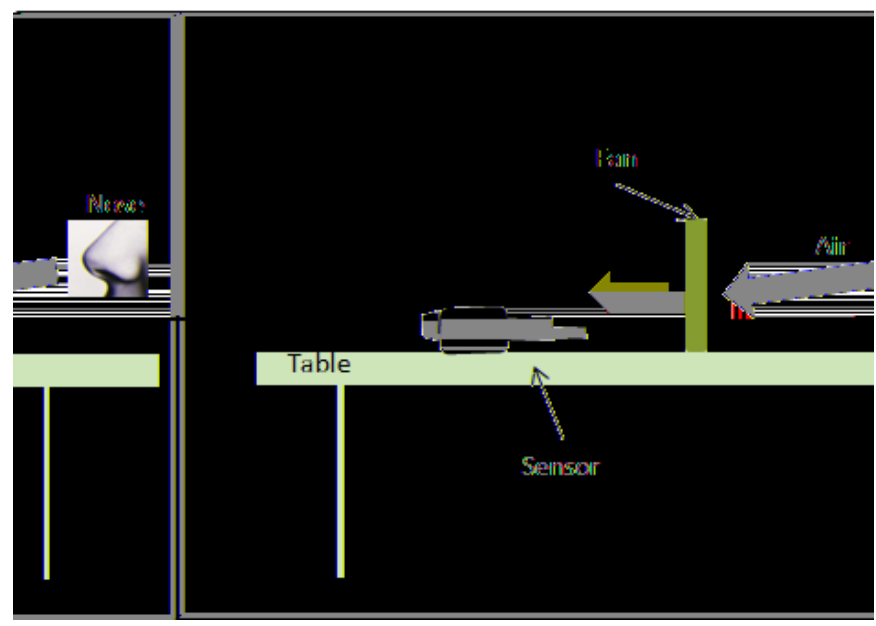

Figure 27 - Visual description of orientation during fan testing

The second set of tests was done using the $80 \mathrm{~mm}$ fan in conjunction with the $40 \mathrm{~mm}$ USB fan. The second fan was added to the configuration seen in Figure 27. It was hypothesized that two fans can further decrease the time it takes for the diffused air to get to the sensor and be pulled off the sensor. It was placed on the other side of the sensor to pull the air off the sensor rapidly. Both fans were tried in each position. The results can be found in the following section and the results are discussed in the section titled "Fan Conclusions."

\section{Fan Testing Results}

The following section will display the results for all of the fan testing that was done to shorten the response time of the $\mathrm{CO}_{2}$ sensor. The results will then be discussed in the "Testing Conclusions and Discussion" section under "Fan Conclusions." The results include testing of fan performance and fan size and are summarized in Table 6-8 and Plots 2-4.

\section{Fan vs. No Fan}

Testing was done to evaluate the effectiveness of an $80 \mathrm{~mm}$ fan coupled with the $\mathrm{CO}_{2}$ sensor. The results in Table 6 are from the tests in which an adult subject held their breath and 
the time it took the sensor to realize breathing had ceased was recorded. It displays the average from three different distances both using the $80 \mathrm{~mm}$ fan to push the air over the sensor and without a fan. Refer back to the "Fan Testing" section and Figure 27 for a description of the testing set up.

Table 6- Average Response times at specified distances

\begin{tabular}{|clc|}
\hline Distance (ft) & \multicolumn{2}{c|}{ Average Response Time (sec) } \\
& With Fan & Without Fan \\
\hline 1 & 11.03 & 29.43 \\
2 & 13.87 & 46.31 \\
3 & 20.52 & 61.49 \\
\hline
\end{tabular}

Minitab was used to statistically analyze the data collected. A boxplot was created for the distance of one foot and can be seen in

Plot 2. The mean response time using the fan was 11.03 seconds and without the fan was 29.43 seconds. A Two Sample T Test was performed to look for significance. A P-value of 0.000 was calculated. Using a confidence interval of $95 \%$ and with a P-value less than 0.05 , statistical significance can be concluded. The $80 \mathrm{~mm}$ fan did reduce the time it took the sensor to recognize the simulated apnea event. 


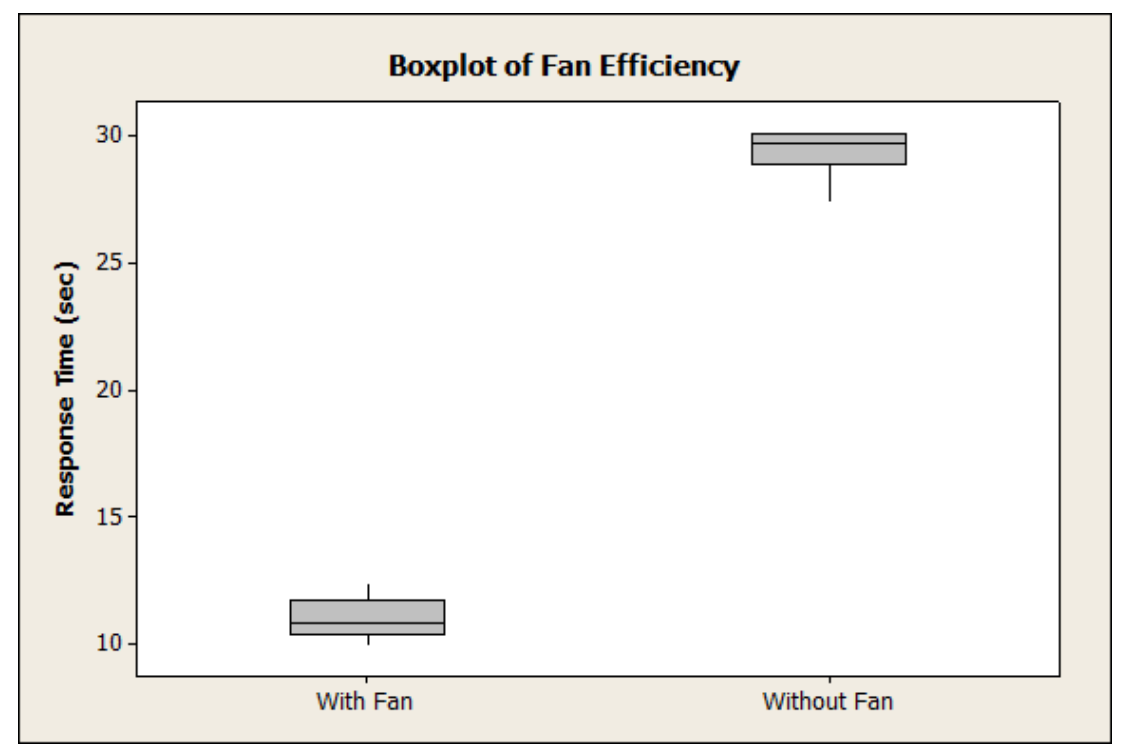

Plot 2 - Boxplot of response time with and without a fan

\section{$80 \mathrm{~mm}$ vs. $40 \mathrm{~mm}$ Fan}

Testing was conducted to compare the $40 \mathrm{~mm}$ fan with the $80 \mathrm{~mm}$ fan. The fans were tested at three different distances and the results are presented in Table 7. At all three distances the $80 \mathrm{~mm}$ fan had a faster response time than the $40 \mathrm{~mm}$ fan.

Table 7 - Average Response Times for both fans

\begin{tabular}{|ccc|}
\hline Distance (ft) & $\begin{array}{c}\text { Average Response Time (sec) } \\
\mathbf{8 0} \mathbf{~ m m}\end{array}$ & \begin{tabular}{c}
$\mathbf{4 0} \mathbf{~ m m}$ \\
\hline
\end{tabular} \\
\hline \hline 1 & 10.67 & 12.42 \\
2 & 14.32 & 17.08 \\
3 & 20.23 & 26.47 \\
\hline
\end{tabular}

The boxplot in

Plot 3 was created to see the differences in response time between the two fans at one foot. Both fans greatly reduce the response of the sensor. Plot 3 gives a visual representation of the $80 \mathrm{~mm}$ fan having a faster response time than both the $40 \mathrm{~mm}$ fan and no fan. Seeing that the $80 \mathrm{~mm}$ fan appeared to aid in a faster response time, statistical analysis was performed to see if 
they were statistically different. Using Minitab, a Two Sample T test was conducted on the 80 $\mathrm{mm}$ fan and the $40 \mathrm{~mm}$ fan at a distance of one foot to get a p-value. A p-value of 0.000 was obtained. This meant that the response time of the $80 \mathrm{~mm}$ fan produces a statistically faster response time than that of the $40 \mathrm{~mm}$ fan. These results will be discussed in the section titled "Fan Conclusions."

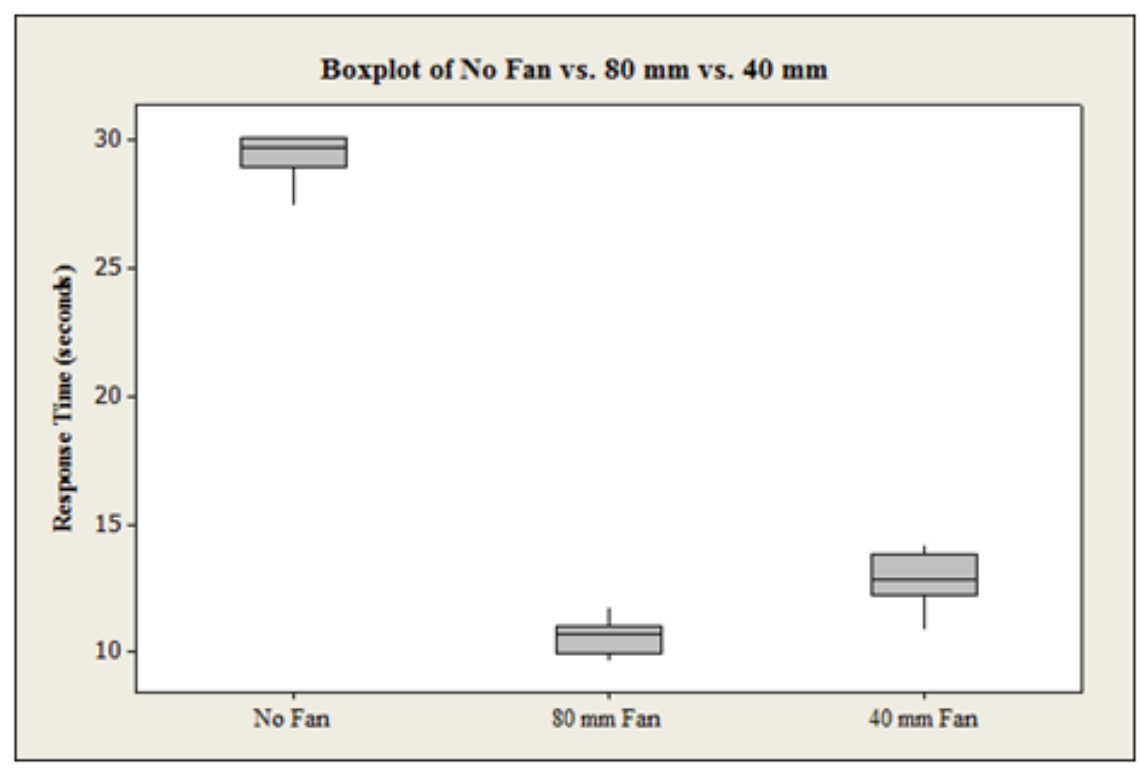

Plot 3 - Boxplot of mean response time, in seconds, for each fan size

\section{Both Fans vs. Single $80 \mathrm{~mm}$ Fan}

Testing was done to see if the response time could further be shortened placing both fans on either side of the sensor. Table 8 shows the results of both fans compared with the results from the $80 \mathrm{~mm}$ fan. For this test the sensor and fan were placed one foot from the subject's nose. Refer back to the "Fan Testing" section for more detail on the testing. 
Table 8 - Results from Testing Both Fans vs. Single 80 mm Fan

\begin{tabular}{|c|cc|}
\hline \multirow{2}{*}{ Test Number } & \multicolumn{2}{|c|}{ Response Times (seconds) } \\
\cline { 2 - 3 } & Both Fans & Single 80 mm fan \\
\hline 1 & 11.85 & 9.97 \\
2 & 10.43 & 10.19 \\
3 & 11.39 & 10.7 \\
4 & 9.79 & 11.05 \\
5 & 10.89 & 9.67 \\
6 & 12.08 & 11.75 \\
7 & 10.75 & 10.82 \\
8 & 10.87 & 11.03 \\
9 & 10.21 & 10.67 \\
10 & 12.45 & 9.89 \\
\hline
\end{tabular}

The average response time for both fans was 11.071 seconds and 10.574 seconds for both fans and the single $80 \mathrm{~mm}$ fan, respectively. The boxplot in

Plot 4 shows that both fans, working together, had a slightly slower response time.

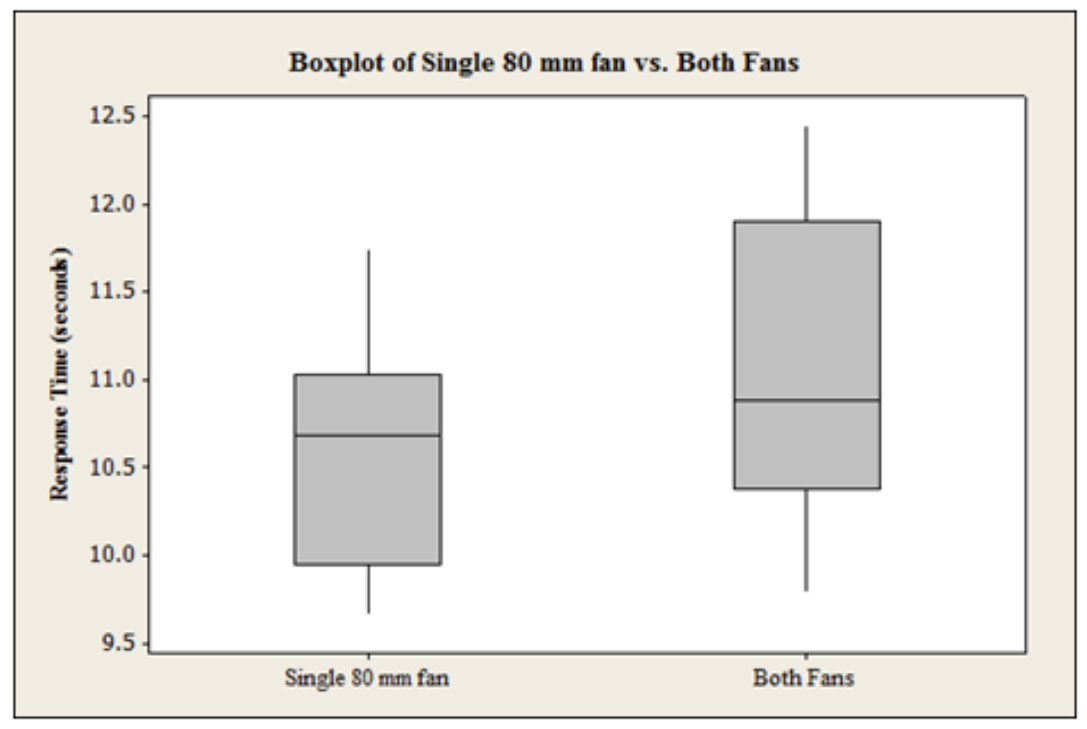

Plot 4 - Boxplot of Both Fans vs. Single 80 mm Fan

Minitab was used to look for a statistically significant difference between the two variables. In conducting a Two Sample T Test a p-value of 0.161 was obtained. This shows that 
while the single $80 \mathrm{~mm}$ fan may have a mean response time that is shorter, it is not statistically significantly different from both fans running together. The implications of these results will be discussed in the section titled "Fan Conclusions."

\section{Enclosure Testing}

Carbon dioxide sensors work on the basis of diffusion and saturation. Infants have a very small lung volume making it hard to collect real time data. It is important to saturate the exhaled air around the sensor as fast as possible. A 4 inch diameter, 6 inch long, white Delrin tube was used for the enclosure surrounding the sensor and fan. It was used because it was readily available in the lab and did not require purchasing any extra components. The tested hypothesis was that the enclosure would help funnel the air over the $\mathrm{CO}_{2}$ sensor, decreasing the response time of the sensor. The enclosure would also act as a way to house all the components of the $\mathrm{CO}_{2}$ detection system.

The enclosed $\mathrm{CO}_{2}$ detection system, seen in Figure 16, was tested on an adult at distances of one and two feet. The test was set up similar to Figure 27, using the enclosure in place of the individual components. Five tests were run at both distances, each running for one minute. During the first 30 seconds the subject breathed normally to establish the baseline. The subject was then told to hold their breath for 15 seconds and then continue normal breathing. The time it took for the $\mathrm{CO}_{2}$ sensor to detect the simulated apnea event was recorded as response time.

After the tests were complete the data was compared to the data collected from the 80 $\mathrm{mm}$ fan testing. Minitab was used to perform statistical analysis and to determine if the results from each test were statistically different. The results from all enclosure testing can be found in the following section. 


\section{Enclosure Results}

Table 9 displays the data collected from the enclosure testing. The results displayed are from the tests that were done one foot from the subject. The average response time using the enclosure was 10.11 seconds and a standard deviation of 0.53 seconds. Without the enclosure the mean response time was 10.57 seconds with a standard deviation of 0.64 seconds. The boxplot in Plot 5 shows that the enclosure had a slightly faster mean response time compared to no enclosure.

Table 9- Results from Enclosure Testing from One Foot

\begin{tabular}{|c|cc|}
\hline \multirow{2}{*}{ Test Number } & \multicolumn{2}{|c|}{ Response Times (seconds) } \\
\cline { 2 - 3 } & Enclosure & No Enclosure \\
\hline 1 & 9.83 & 9.97 \\
2 & 9.74 & 10.19 \\
3 & 9.29 & 10.7 \\
4 & 10.19 & 11.05 \\
5 & 10.4 & 9.67 \\
6 & 10.07 & 11.75 \\
7 & 10.68 & 10.82 \\
8 & 9.56 & 11.03 \\
9 & 10.34 & 10.67 \\
10 & 11.03 & 9.89 \\
\hline
\end{tabular}

Table 10 shows the results from the enclosure testing at distance of two feet. The average response time of the $\mathrm{CO}_{2}$ sensor with the enclosure was 12.91 seconds with a standard deviation of 0.77 seconds. The subject only held their breath for 15 seconds, so if the apnea event was not detected within those 15 seconds the result was recorded as "Not Detected." The average response time at two feet, without the enclosure was 14.10 seconds with a standard deviation of 0.66 seconds, for the values that were recorded. 
Table 10 - Enclosure Response Time from Two Feet

\begin{tabular}{|c|cc|}
\hline \multirow{2}{*}{$\begin{array}{c}\text { Test } \\
\text { Number }\end{array}$} & \multicolumn{2}{|c|}{ Response Times (seconds) } \\
\cline { 2 - 3 } & Enclosure & No Enclosure \\
\hline 1 & 13.62 & 14.82 \\
2 & 12.47 & Not Detected \\
3 & 12.36 & 13.74 \\
4 & 11.8 & 14.03 \\
5 & 13.59 & 14.93 \\
6 & 12.87 & Not Detected \\
7 & 13.02 & 13.78 \\
8 & 11.89 & 12.98 \\
9 & 14.03 & 14.64 \\
10 & 13.46 & 13.85 \\
\hline
\end{tabular}

Statistical analysis was done using Minitab. Plot 5 shows a visual representation of the response times from a distance of one foot. A p-value was calculated to see if this difference was statistically significant. The calculated p-value was found to be 0.097 .

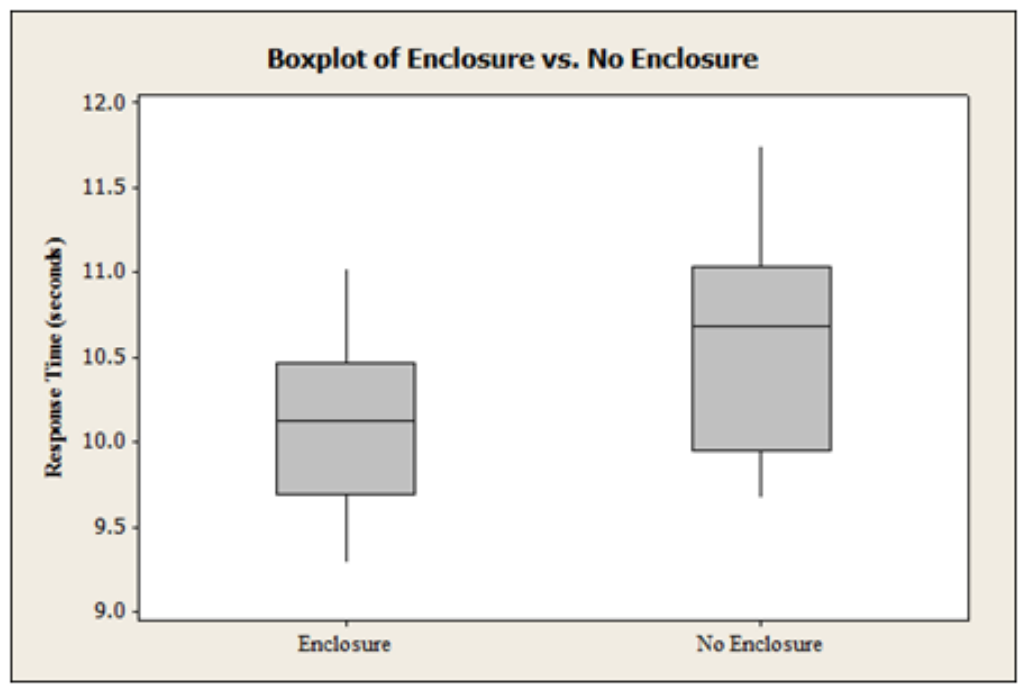

Plot 5 - Boxplot of Enclosure vs. No Enclosure Response Time

The boxplot in Plot 6 shows a visual representation of the response time of the $\mathrm{CO}_{2}$ sensor from two feet. The enclosure appears to aid in decreasing the response time. To determine if this difference is statistical a p-value was obtained. The p-value at two feet was 0.242. 


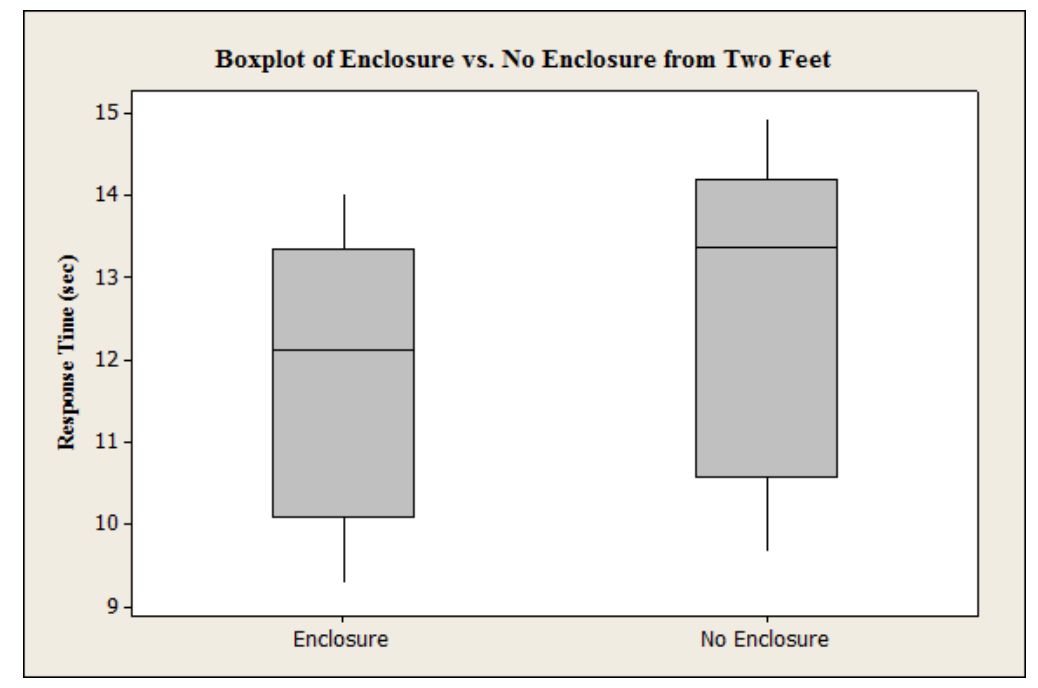

Plot 6 - Boxplot of Enclosure Testing from Two Feet

Both p-values were greater than 0.05, showing that the shortened response time using the enclosure is not statistically significant. These results will be discussed in the section titled "Enclosure Conclusions."

\section{Infant Testing}

Once an acceptable response time was obtained from the $\mathrm{CO}_{2}$ sensor, it was tested on infants. The monitor was meant to track changes in infant breath, so this testing was extremely valuable. The contact we had for infant testing was at Cottage Hospital in Goleta, CA. This limited the amount of infant testing we could do. The audio system was tested alongside the $\mathrm{CO}_{2}$ system, so some audio results will also be presented. The following section will detail the testing that was done and display the results in Tables 12-14.

\section{NICU Testing}

Testing in the NICU was essential to this thesis due to the objective of the project. The objective of this thesis was to design and test a standoff infant apnea monitor, which meant testing on infants was essential. Two separate trips were made down to Cottage Hospital in 
Goleta, CA to acquire information from Dr. Barkley and test the device on the infants in the NICU.

The first trip was for research purposes. Dr. Barkley instructed us on his experience with infant apnea, infant physiology, and showed us some of their current equipment used to monitor infants with apnea in the NICU. The information we gained was used to narrow the scope of the project and define some of the constraints that were discussed in previous sections.

The second trip was taken in June 2009, at the end of the project. The purpose of this trip was to test the proof-of-concept standoff infant apnea monitor we had designed. Dr. Barkley had preselected three infants that we could use to gain data. They were full term infants that did not have any preexisting medical conditions and had shown signs of apnea during their stay in the NICU. Dr. Barkley was not able to observe or participate in the testing due to other infants in need of his attention.

Before taking readings from any infants we first took three readings of the ambient carbon dioxide levels and audio. This was to ensure the system was working properly and the values could be used to compare with the values obtained from the infants. Three tests of five minutes each were run to monitor the ambient environment and make any changes to the settings in the infant apnea detection software.

We then moved to the back of the NICU where Dr. Barkley had an infant in a hospital bassinet. For this test a predetermined protocol had been set and can be viewed in Table 11. Three tests were conducted. Each test consisted of running the prototype monitor for five minutes at a distance of 6 inches, one foot, and two feet. Only one of the infants was available for testing due to visits from friends and family. The results from these tests can be found in the following section and will be discussed in the section titled "NICU Testing Conclusions." 
Table 11 - Protocol for NICU testing

\begin{tabular}{|ccc|}
\hline $\begin{array}{c}\text { Test } \\
\text { Number }\end{array}$ & Duration & $\begin{array}{c}\text { Distance from } \\
\text { Infant }\end{array}$ \\
\hline 1 & $5 \mathrm{~min}$. & $6 \mathrm{in}$ \\
2 & $5 \mathrm{~min}$. & $1 \mathrm{ft}$ \\
3 & $5 \mathrm{~min}$. & $2 \mathrm{ft}$ \\
\hline
\end{tabular}

The audio detection system was run alongside the $\mathrm{CO}_{2}$ system, but was run on a separate laptop. The protocol was the same for the audio testing. In between each test one minute of infant breath sounds were recorded for future analysis. The recorded sound was used for frequency analysis to look at how many breaths were being picked up by the microphone and at what frequency the infant breath was being heard by the microphone.

\section{NICU Results}

The following sections display the results that were obtained from the infant testing that was done at Cottage Hospital in Goleta. The infant on which the testing was conducted did not have any apnea events throughout the duration of our testing. This was confirmed by the hospital pulse oximeter and the heart rate monitor that was on the infant during testing. The results presented will be focused on the $\mathrm{CO}_{2}$ portion of the testing, although the audio results will be presented. For comprehensive audio detection system results refer to Dan's thesis.

The graph in the front panel of the LabView system can be seen in Figure 28. The graph on the left is from an initial baseline test. The $\mathrm{CO}_{2}$ concentration is staying relatively level so the system detects breath. The graph on the right is from a test using the infant. Although there are fluctuations in the $\mathrm{CO}_{2}$ concentrations, no apnea is detected, due to the way the baseline is established. 


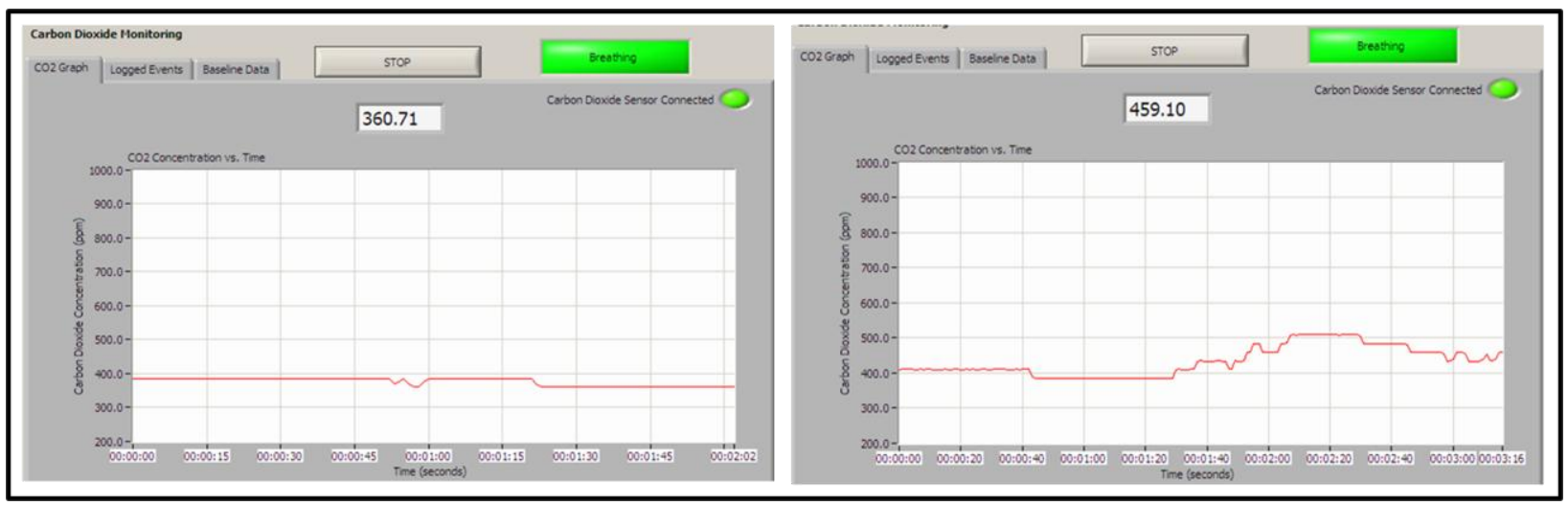

Figure 28 - LabView Front Panel of NICU Results

Table 12 shows the $\mathrm{CO}_{2}$ results that were obtained during infant testing at Cottage

Hospital. Three baseline measurements were first taken of the atmospheric $\mathrm{CO}_{2}$ in the NICU.

The average ambient $\mathrm{CO}_{2}$ concentration in the NICU was $352.25 \mathrm{ppm}$. The average $\mathrm{CO}_{2}$

concentration measured 6 inches from the infant was $434.43 \mathrm{ppm}$. The average $\mathrm{CO}_{2}$

concentration one foot from the infant was $442.1 \mathrm{ppm}$. The last distance tested was two feet from the infant and the average $\mathrm{CO}_{2}$ concentration was $410.63 \mathrm{ppm}$. During testing, the $\mathrm{CO}_{2}$ concentration fluctuates. This is seen by the column displaying the range of $\mathrm{CO}_{2}$ values measured. These averages can be seen in the Individual Value Plot in Plot 7. The plot shows that the baseline $\mathrm{CO}_{2}$ concentrations were lower that the concentrations measured around the infant.

Table 12 - Results from NICU Testing

\begin{tabular}{|c|c|c|c|c|}
\hline Test & $\begin{array}{c}\text { False } \\
\text { Alarms }\end{array}$ & $\begin{array}{c}\text { CO2 } \\
\text { Range } \\
(\mathbf{p p m})\end{array}$ & $\begin{array}{c}\text { Average } \\
\mathbf{C O 2} \\
(\mathbf{p p m})\end{array}$ & $\begin{array}{c}\text { Duration of } \\
\text { Test }\end{array}$ \\
\hline 1st Baseline & 0 & $350-410$ & 363.65 & $10 \mathrm{~min}$ \\
\hline 2nd Baseline & 0 & $330-410$ & 338.38 & $10 \mathrm{~min}$ \\
\hline 3rd Baseline & 0 & $350-390$ & 360.71 & $2 \mathrm{~min} 2 \mathrm{sec}$ \\
\hline $\begin{array}{c}\text { 6 inches away } \\
\text { from infant }\end{array}$ & 0 & $420-490$ & 434.43 & $5 \mathrm{~min} 34 \mathrm{sec}$ \\
\hline $\begin{array}{c}\text { 1 foot away } \\
\text { from infant }\end{array}$ & 1 & $390-475$ & 442.1 & $3 \mathrm{~min} 16 \mathrm{sec}$ \\
\hline $\begin{array}{c}\text { 2 feet away } \\
\text { from infant }\end{array}$ & 0 & $380-435$ & 410.63 & $5 \mathrm{~min} 57 \mathrm{sec}$ \\
\hline
\end{tabular}




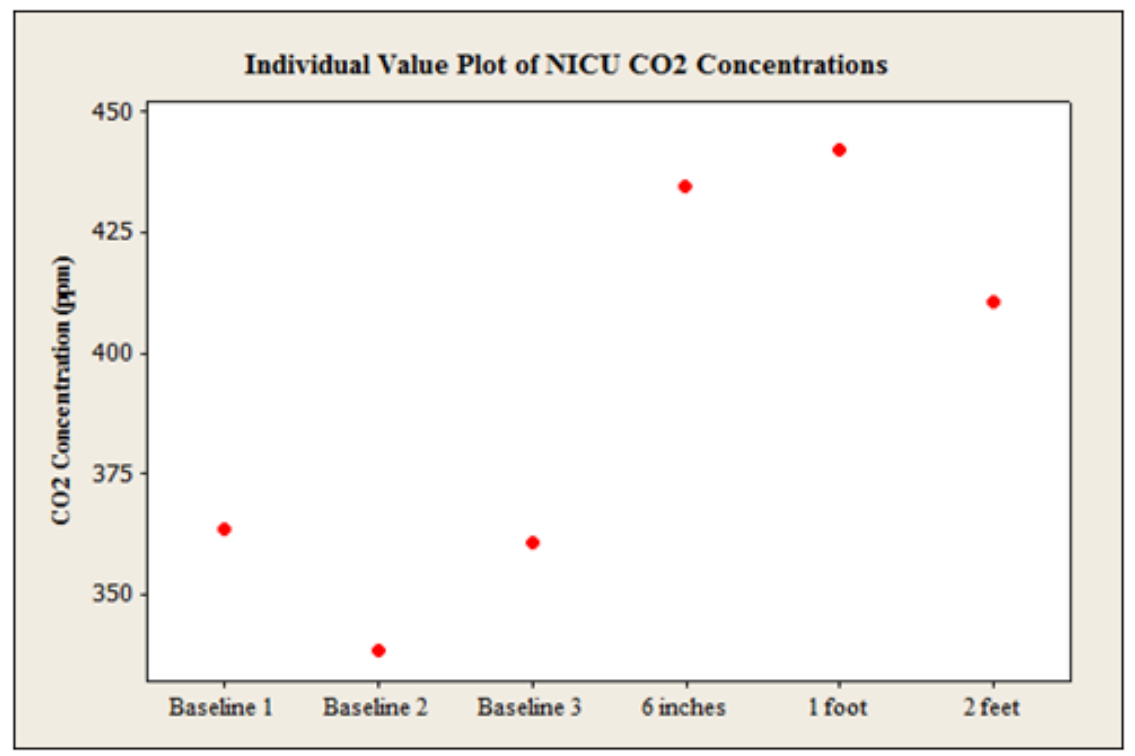

Plot 7- Individual Value Plot of NICU Results

The baseline values were compared to the concentrations measured at each distance to look for statistical difference. The p-values can be found in Table 13. Each distance was compared to the baseline values using a Two Sample T Test. All three distances had $\mathrm{CO}_{2}$ concentration readings that were statistically higher than the baseline. This indicated breath was detected by the $\mathrm{CO}_{2}$ detection system at all three distances.

Table 13 - P-values from NICU Testing

\begin{tabular}{|lc|}
\hline Distance & p-value \\
\hline 6 inches & 0.01 \\
1 foot & 0.008 \\
2 feet & 0.019 \\
\hline
\end{tabular}

The audio detection system was simultaneously being run alongside the $\mathrm{CO}_{2}$ detection system. All three distance were tested and results can be found in Table 14. After each test, sound from the infant was recorded for future analysis, as denoted by $1 \mathrm{~b}, 2 \mathrm{~b}, 3 \mathrm{~b}$. No apnea events were detected at 6 inches. At one foot, 3 apnea events were detected and 8 near apnea events were detected. The near apnea events correspond to no breath being detected for a 
specified fraction of the 20 seconds of a defined apnea event. For this test, if no breath was detected for 10 seconds a near apnea event was recorded.

Table 14 - Audio results from Infant Testing

\begin{tabular}{|c|c|c|c|c|}
\hline Test \# & Duration & Distance & Apneas & Near Apneas \\
\hline $1 \mathrm{a}$ & $5 \mathrm{~min}$ & 6 in & 0 & 0 \\
\hline $1 \mathrm{~b}$ & $1 \mathrm{~min}$ & 6 in & \multicolumn{2}{|c|}{ (sound recorded) } \\
\hline $2 \mathrm{a}$ & $5 \mathrm{~min}$ & $12 \mathrm{in}$ & 3 & 8 \\
\hline $2 \mathrm{~b}$ & $1 \mathrm{~min}$ & $12 \mathrm{in}$ & \multicolumn{2}{|c|}{ (sound recorded) } \\
\hline $3 \mathrm{a}$ & $5 \mathrm{~min}$ & 24 in & $0^{*}$ & $0^{*}$ \\
\hline $3 \mathrm{~b}$ & $1 \mathrm{~min}$ & 24 in & \multicolumn{2}{|c|}{ (sound recorded) } \\
\hline
\end{tabular}

The audio that was recorded between tests was used for frequency analysis of the breath in a separate program that was not a part of the system designed for this project. A visual representation of the frequency analysis can be found in Figure 29. This is from the sounds recorded at 6 inches. Every red dot indicates visualized breath. When compared to the rate of infant breath, 25-50 breaths per minute, the audio detection system "heard" approximately 50\% of the breaths. These results will be discussed in the section titled "NICU Testing Conclusions."

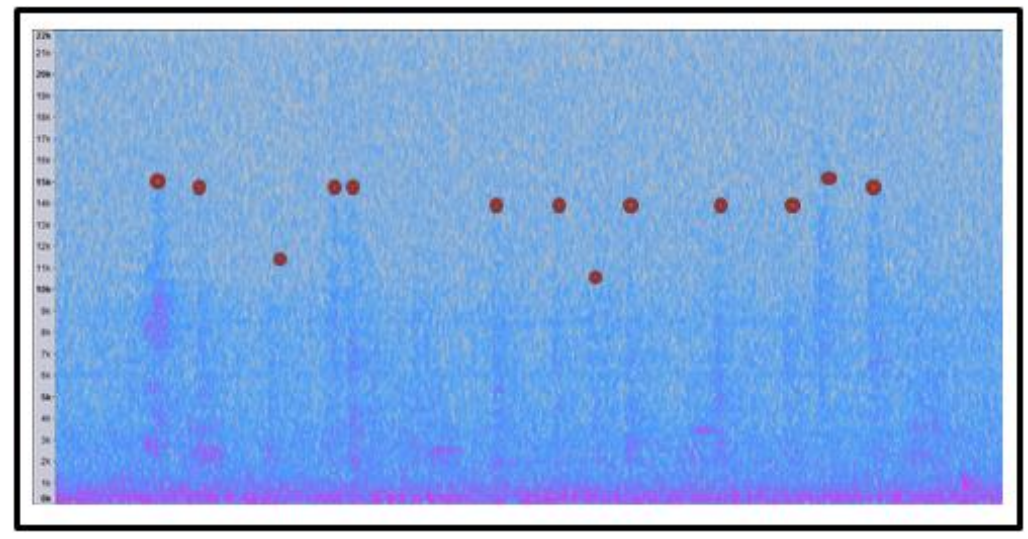

Figure 29 - Frequency Analysis of Infant Breath 


\section{Overnight Testing}

Testing in the NICU was limited due to the schedules of those involved in this project at Cal Poly and the staff of the NICU at Cottage Hospital. It was concluded that for this prototyping stage of the project, adult testing could be done to gain data when infant testing was not available.

To collect long term data, overnight testing was conducted on adults. It was not possible for us to do the overnight testing on the infants in the NICU at Cottage Hospital due to hospital policy and logistics that would go along with being down in Goleta for a long period of time. Long term data collection is important to ensure that there are no problems with the systems if they are run for many hours and to get realistic results using the device in an atmosphere in which it would likely be used. The final product is intended to be used in an overnight setting while the infant slept so this testing gave us important information. The overnight testing was done on an adult who was known to have no form of adult sleep apnea. Any detected apnea event was considered false alarms.

With the software we developed in LabView we were able to use the "Logged Events" portion of each system, $\mathrm{CO}_{2}$ and audio, and look at them independently as well as combined to determine if a false apnea event had been detected. Each system logs events that it detects independently from the other and the combined system logs events that are triggered by both detection systems.

One adult was used for the testing and the tests were conducted on 26 different nights. For the first 15 nights only the $\mathrm{CO}_{2}$ detection system was tested because the student working on the audio portion was in possession of the hardware during the time of the testing. The last 11 nights, both the audio and $\mathrm{CO}_{2}$ systems were combined for whole device testing. It is important 
to reiterate that the adult that the tests were conducted on was known to not have any form of apnea, so any events that were recorded would indicate a false positive.

\section{Overnight Tests with $\mathrm{CO}_{2}$ System Alone}

As stated previously, the first 15 overnight tests were done using only the $\mathrm{CO}_{2}$ system. The tests were conducted with the sensor mounted in three different orientations as listed in Table 15. The testing was done in the bottom bunk of a bunk bed. The bed was placed next to a window sill. In orientation $\mathrm{A}$ the $\mathrm{CO}_{2}$ system was placed on the window sill pointed towards the bed. The subject fell asleep on their left side, facing the sensor, but may have changed positions throughout the night. In orientation $\mathrm{B}_{1}$ the $\mathrm{CO}_{2}$ system was mounted to the underside of the top bunk of the bunk bed, two feet from the subject's face. The subject fell asleep lying on their back. Orientation $\mathrm{B}_{2}$ was the same as $\mathrm{B}_{1}$, but lowered so the $\mathrm{CO}_{2}$ system was one foot from the subject's face. The subject also fell asleep lying on their back in this orientation. Testing in all three configurations would allow comparisons to see how they differed and which configuration was most accurate and would log the least number of false alarms throughout the course of a night. Breathing solely out of the nose was not taken into account, because the subject was asleep and that variable could not be controlled. 
Table 15 - Orientation of CO2 system during overnight testing

\begin{tabular}{|c|l|}
\hline Orientation & \multicolumn{1}{|c|}{ Description } \\
\hline \hline $\mathrm{A}$ & $\begin{array}{l}\text { CO2 device sits on the window sill } \\
\text { Fell asleep laying on left side facing the window sill } \\
\text { Distance from device to face about } \mathbf{2} \mathbf{f t}\end{array}$ \\
\hline $\mathrm{B}_{1}$ & $\begin{array}{l}\text { Device mounted above bed and directly above head, pointing } \\
\text { straight down at person while they sleep } \\
\text { Distance from device to face about } \mathbf{2} \mathbf{f t}\end{array}$ \\
\hline $\mathrm{B}_{2}$ & $\begin{array}{l}\text { Device mounted above bed and directly above head, pointing } \\
\text { straight down at person while they sleep } \\
\text { Distance from device to face about } \mathbf{1} \mathbf{f t}\end{array}$ \\
\hline
\end{tabular}

Depending on the orientation the device was either placed on the window sill or directly above the subject while they slept. The Netbook used was small enough to be placed on the window sill. The first night the Netbook went into hibernate mode after one hour, so the data for that night was only used after the false apnea events were normalized per hour. The settings on the computer were changed and the remaining 14 tests ran smoothly.

The test was run for an average of 7 hours each night. The first five nights the test was run in orientation $\mathrm{A}$. The next five nights the test was run in orientation $\mathrm{B}_{1}$. The last four nights the test was run in orientation $B_{2}$. The program was started when the subject climbed into bed and ran throughout the night. When the subject awoke the next morning the program was stopped and the data was collected. The raw data of runtime, total apnea events logged, and events per hour were inputted into an Excel spreadsheet for further investigation.

Statistical analysis was conducted using Excel and Minitab. The results from each orientation in Table 15 were compared to each other to look for statistically significant differences. The logged events were considered false positives. These results can be found in the following section. 


\section{Results from $\mathrm{CO}_{2}$ Overnight Tests}

Table 16 shows the data recorded from the 15 overnight trials. In device orientation A the sensor was placed on the window sill approximately 2 feet to the left of the adult. In orientation $\mathrm{B}_{1}$ the device was mounted above the bed 2 feet from the subject. In orientation $\mathrm{B}_{2}$ the device was mounted the same as $B_{1}$ but lowered to 1 foot above the subject. Each night the run time of the sensor was recorded, as well as the number of detected apnea events. They were divided to normalize the data to false alarms per hour. The first night's data could then be used because the system had run for one hour.

Table 16 - Data recorded from 15 overnight tests

\begin{tabular}{|c|c|c|c|c|}
\hline Date & $\begin{array}{c}\text { Run Time } \\
\text { (hrs) }\end{array}$ & $\begin{array}{c}\text { Apnea Events } \\
\text { Logged }\end{array}$ & $\begin{array}{c}\text { False Alarms per } \\
\text { Hour }\end{array}$ & Device orientation \\
\hline $10 / 11 / 2009$ & 1 & 3 & 3.00 & $\mathrm{~A}$ \\
\hline $10 / 13 / 2009$ & 7 & 26 & 3.71 & $\mathrm{~A}$ \\
\hline $10 / 14 / 2009$ & 6.5 & 21 & 3.23 & $\mathrm{~A}$ \\
\hline $10 / 16 / 2009$ & 8 & 24 & 3.00 & $\mathrm{~A}$ \\
\hline $10 / 17 / 2009$ & 7 & 22 & 3.14 & $\mathrm{~A}$ \\
\hline $10 / 19 / 2009$ & 6 & 18 & 3.00 & $\mathrm{~B}_{1}$ \\
\hline $10 / 21 / 2009$ & 6 & 14 & 2.33 & $\mathrm{~B}_{1}$ \\
\hline $10 / 22 / 2009$ & 7 & 15 & 2.14 & $\mathrm{~B}_{2}$ \\
\hline $10 / 35 / 2009$ & 7 & 10 & 1.43 & $\mathrm{~B}_{1}$ \\
\hline $10 / 26 / 2009$ & 8 & 17 & 2.13 & $\mathrm{~B}_{1}$ \\
\hline $10 / 28 / 2009$ & 7.5 & 13 & 1.73 & $\mathrm{~B}_{2}$ \\
\hline $10 / 29 / 2009$ & 8 & 12 & 1.50 & $\mathrm{~B}_{1}$ \\
\hline $10 / 30 / 2009$ & 8.5 & 15 & 1.76 & $\mathrm{~B}_{2}$ \\
\hline $11 / 1 / 2009$ & 6.5 & 10 & 1.54 & $\mathrm{~B}_{2}$ \\
\hline $11 / 2 / 2009$ & 5.5 & 12 & 2.18 & \\
\hline
\end{tabular}

Plot 8 is a boxplot comparing the three difference orientations used to test the $\mathrm{CO}_{2}$ apnea detection system. Orientation A had a mean detected apnea events per hour of 3.18. Orientation $\mathrm{B}_{1}$ had a mean detected apnea events per hour of 2.02. Orientation $\mathrm{B}_{2}$ had a mean detected apnea 
events per hour of 1.66. A Paired $\mathrm{T}$ test was performed on orientation $\mathrm{A}$ and orientation $\mathrm{B}_{1}$ to obtain a $\mathrm{p}$-value of 0.001 . This $\mathrm{p}$ value indicates that that orientation $\mathrm{A}$ and $\mathrm{B}_{1}$ are statistically different. A two sample T test was performed on orientation A vs. $\mathrm{B}_{2}$ because the samples were not of equal size. A p-value of 0.001 was calculated showing that orientation $\mathrm{A}$ and $\mathrm{B}_{2}$ are also statistically different. A two sample T test was also performed on orientation $\mathrm{B}_{1} \mathrm{vs}$. $\mathrm{B}_{2}$ and a pvalue of 0.287 was calculated showing that orientation $B_{1}$ and $B_{2}$ were statistically similar.

Conclusions that can be drawn from these results will be discussed in the section titled " $\mathrm{CO}_{2}$ Conclusions."

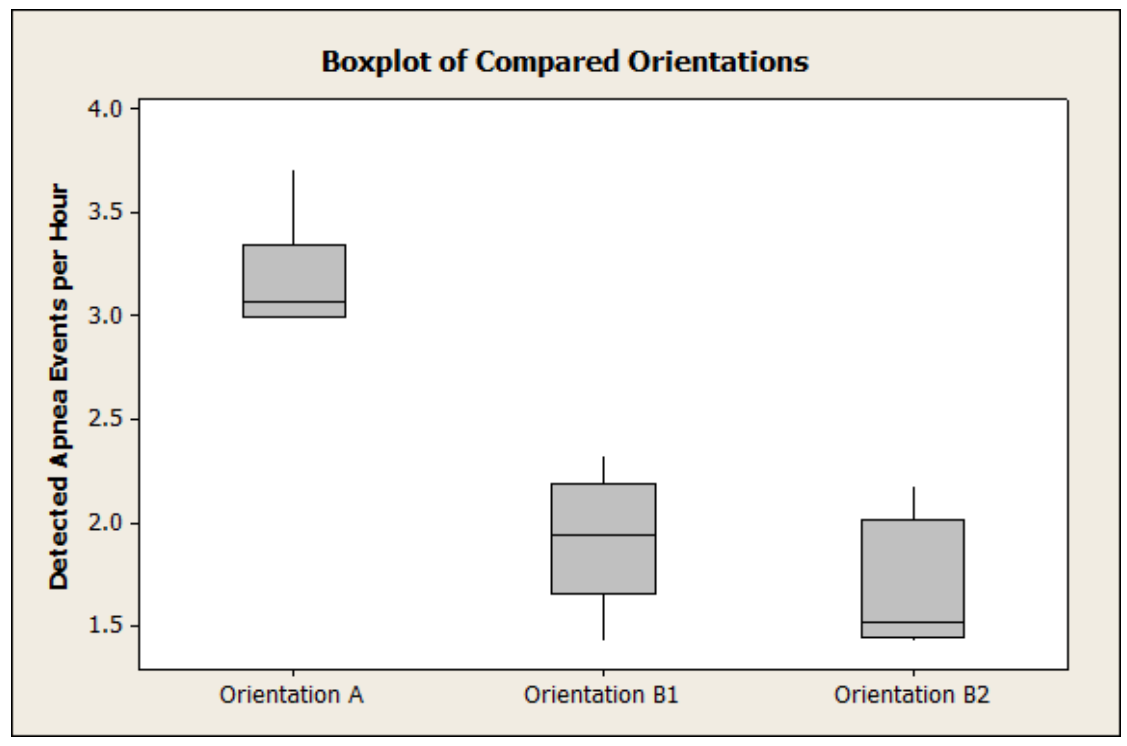

Plot 8 - Boxplot of Compared Orientations

\section{Overnight Testing of Combined System}

This section will discuss the methods used to conduct overnight tests on an adult subject using both the $\mathrm{CO}_{2}$ and the audio detection systems. These tests were conducted to see if there were any interactions between the systems if they were run all night. The results can be found in 
the following section. It is important to note that the subject used for this test did not have any form of sleep apnea, so any detected apnea events would be logged as false alarms.

The first night the detections systems were mounted next to each other, which may have caused the sound of the fan to interfere with the audio detection system. To ensure that the noise from the fan no longer interfered with the audio apnea detection system they were separated. The directional microphone was placed on the window sill, one foot away from the subject's face. The omni-directional microphone was placed on the side of the bed facing out into the room. The $\mathrm{CO}_{2}$ system was mounted in the $\mathrm{B}_{2}$ orientation from Table 15 . A total of 11 overnight tests were run using the same subject as was used for the overnight $\mathrm{CO}_{2}$ testing. The test was run an average of 6.45 hours each night. Each night the total number of detected apnea events was recorded for each system separately and combined. The number of apnea events detected was also broken down by hour for analysis. The results were put into Table 17 using Excel and can be found in the following section.

Statistical analysis was conducted using Excel and Minitab. A boxplot was created to show the difference in number of detected apnea events per hour for each system and the combined system. The detection systems were also compared to each other and the combined system. These results can also be found in the following section.

If conclusions can be drawn from the results and statistical analysis, I will do so. The implications from all of the overnight testing will be discussed in the section titled "Overnight Testing Conclusions and Discussion”

\section{Results of Integrated Systems Testing}

This section will discuss the results that were obtained from running overnight test using the finished proof-of-concept standoff infant apnea monitor. Only results will be presented in this 
section and a discussion of the results can be found in the section titled "Overnight Testing Conclusions."

Table 17 displays the data collected from the 11 overnight tests run with the integrated system. Each system has its own log, so individual events were recorded as well as events that were detected with both systems. These results were also normalized to apnea events detected per hour. The mean run time for the tests was 6.45 hours. The $\mathrm{CO}_{2}$ system alone detected an average of 2.98 apnea events per hour. The audio system alone detected an average of 1.02 apnea events per hour. The combined system detected an average of 0.88 apnea events per hour. The first night the audio and $\mathrm{CO}_{2}$ detection systems were placed next to each other, so the audio detection system may have not detected any apnea events because the fan of the $\mathrm{CO}_{2}$ detection system was too loud. It is important to remember that all of the detected events are considered false apnea events because the test subject does not have any form of apnea.

Table 17 - Logged Apnea Events for overnight testing

\begin{tabular}{|c|c|c|c|c|c|c|c|}
\hline & \multicolumn{2}{|c|}{ Apnea Events Logged } & \multicolumn{3}{c|}{ Apnea Events per Hour } \\
\hline Date & $\begin{array}{c}\text { Run } \\
\text { Time } \\
\text { (hrs) }\end{array}$ & $\begin{array}{c}\text { CO2 } \\
\text { Alone }\end{array}$ & $\begin{array}{c}\text { Audio } \\
\text { Alone }\end{array}$ & Combined & $\begin{array}{c}\text { CO2 } \\
\text { Alone }\end{array}$ & $\begin{array}{c}\text { Audio } \\
\text { Alone }\end{array}$ & Combined \\
\hline $11 / 3 / 2009$ & 7 & 21 & 0 & 0 & 3 & 0 & 0 \\
\hline $11 / 4 / 2009$ & 6 & 19 & 5 & 4 & 3.17 & 0.83 & 0.67 \\
\hline $11 / 5 / 2009$ & 7.5 & 23 & 8 & 8 & 3.07 & 1.07 & 1.07 \\
\hline $11 / 9 / 2009$ & 8 & 27 & 12 & 8 & 3.38 & 1.50 & 1.00 \\
\hline $11 / 10 / 2009$ & 6 & 16 & 6 & 6 & 2.67 & 1.00 & 1.00 \\
\hline $11 / 11 / 2009$ & 6.5 & 17 & 5 & 5 & 2.62 & 0.77 & 0.77 \\
\hline $11 / 17 / 2009$ & 5 & 16 & 4 & 4 & 3.20 & 0.80 & 0.80 \\
\hline $11 / 19 / 2009$ & 7 & 20 & 6 & 4 & 2.86 & 0.86 & 0.57 \\
\hline $11 / 21 / 2009$ & 7 & 22 & 11 & 8 & 3.14 & 1.57 & 1.14 \\
\hline $11 / 22 / 2009$ & 6 & 14 & 6 & 6 & 2.33 & 1.00 & 1.00 \\
\hline $11 / 25 / 2009$ & 5 & 17 & 4 & 4 & 3.40 & 0.80 & 0.80 \\
\hline
\end{tabular}


Minitab was used for statistical analysis of the results in Table 17. The boxplot in Plot 9 was created for a visual representation of the average apnea events per hour for $\mathrm{CO}_{2}$, audio, and integrated detection systems.

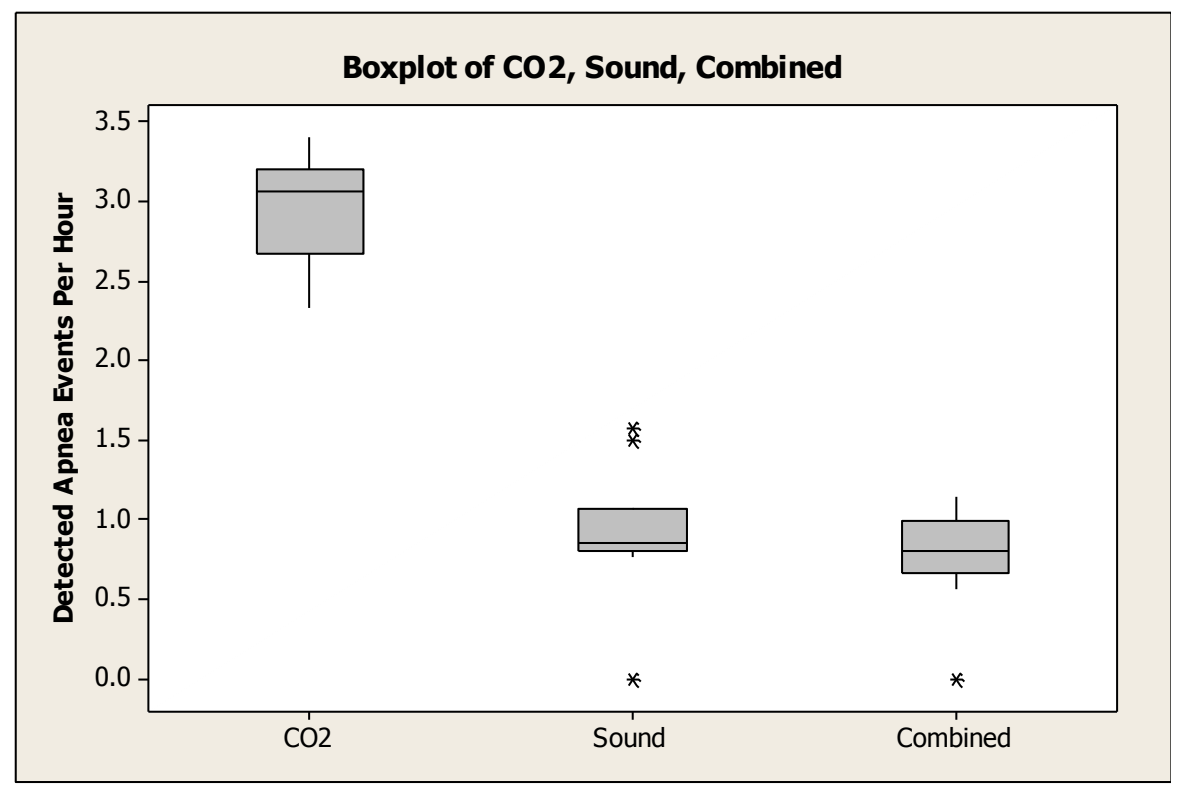

Plot 9 - Boxplot of Apnea Detection Systems

Using a Two Sample T Test all three scenarios were compared to each other to see if the differences in detected apnea events were statistically significant. The p-values can be found in Table 18. The number of apnea events detected with the sound detection system and the combined system were not statistically different. Combining sound with the $\mathrm{CO}_{2}$ statistically improved the number of false alarms detected over the $\mathrm{CO}_{2}$ system alone.

Table 18 - P-values for overnight testing results

\begin{tabular}{|lr|}
\hline Comparison & P-Value \\
\hline \hline CO2 - Sound & 0.000 \\
Sound - Combined & 0.453 \\
CO2 - Combined & 0.000 \\
\hline
\end{tabular}




\section{Conclusions and Discussions}

The following sections will present the conclusions and interpretations of the all the results obtained during testing. Refer to the "Standoff Apnea Monitor Testing and Results" section to view the results that are discussed in the following sections. Where statistical analysis allows, conclusions will be drawn. The results from the overnight testing can only be apply to monitoring adult apnea because adults are not obligate nose breathers, like infants. Future testing will be discussed in section titled "Future Work and Next Steps."

\section{$\mathrm{CO}_{2}$ Sensor Validation Conclusions}

Before any data can be collected the measurement system must be calibrated and validated. This section will discuss the results from the validation tests that were presented in the " $\mathrm{CO}_{2}$ Sensor Validation" section.

The published value of $388.63 \mathrm{ppm}$ was taken from peer-reviewed literature. The measured values in Table 4 were compared to the hypothesized value and a difference between the two was calculated. The differences were displayed in Plot 1 . This was done to ensure the $\mathrm{CO}_{2}$ sensor was accurately measuring atmospheric carbon dioxide before it was tested on human subjects. The hypothesized value of $388.63 \mathrm{ppm}$ fell within a 95\% confidence interval of 387.89 and $394.31 \mathrm{ppm}$. This means that $95 \%$ of the time a measured atmospheric $\mathrm{CO}_{2}$ value will fall between those two values. A p-value greater than 0.05 showed that there was no statistical difference between the measured $\mathrm{CO}_{2}$ values and the hypothesized $\mathrm{CO}_{2}$ value. With the $\mathrm{CO}_{2}$ sensor validated testing could proceed as planned. 


\section{Solo $\mathrm{CO}_{2}$ Sensor Testing Conclusions}

The results in Table 5 show the response times from the 10 test that were conducted with the sensor alone. The results showed that the sensor could detect an absence of breath. The average response time from one foot away from the adult was 29.43 seconds. An apnea event, in both an infant and an adult, is defined as a cessation of breathing for 20 seconds or longer. These results show that the sensor, by itself, cannot detect a lack of breath within the critical time of 20 seconds.

Without modifications this response time is not acceptable. These modifications were made by adding a fan, an enclosure, and modifications directly to the sensor. These additions will be discussed in the following sections.

\section{Fan Conclusions}

This section will discuss the results of the fan testing that was done. All of the fan testing was conducted on an adult. Simulated apnea events were used to look at response time of the sensor. Refer back to the "Fan Testing" section for a description of how the tests were run. The results were presented in Table 6 through Table 8. When conclusions can be drawn, I will do so. Refer to the "Fan Testing Results" section to see the data collected from the testing.

\section{Single Fan Conclusions}

The first set of tests was done to determine if the $80 \mathrm{~mm}$ fan increased the real time reading of the $\mathrm{CO}_{2}$ sensor. The $80 \mathrm{~mm}$ fan reduced the sensor response time to an average of 11.03 seconds at one foot, 13.87 seconds at two feet, and 20.52 seconds at three feet. This more than cuts in half the 29.43 seconds response time without the fan. A p-value less than 0.05 , led to the conclusion that the $80 \mathrm{~mm}$ fan statistically significantly reduced the response time of the 
sensor at these three distances. Table 6 showed that at a distance of one and two feet the response time was within the 20 seconds that is defined as infant or adult apnea. It is important to keep in mind that these tests were run on adults and results may vary with infants due to their smaller lung volume.

A smaller fan was tested against the $80 \mathrm{~mm}$ fan to determine if the results were altered the by size of the fan. A $40 \mathrm{~mm}$ was used to compare to response time data collected to the 80 $\mathrm{mm}$ fan and the $\mathrm{CO}_{2}$ sensor alone at one, two, and three feet.

Plot 3, in the section titled "Fan Results" shows that the response time of the sensor, with both the $40 \mathrm{~mm}$ and $80 \mathrm{~mm}$ fan, was shorter than without the fan at a distance of one foot from the test subject. The p-value less than 0.05 indicted that the $40 \mathrm{~mm}$ fan also statistically significantly shortened the response time of the sensor. When the $40 \mathrm{~mm}$ fan was statistically compared to the $80 \mathrm{~mm}$ fan a p-value of 0.00 meaning the differences in response time were statistically different. Although the $40 \mathrm{~mm}$ fan also produced response times within the 20 second limit, the $80 \mathrm{~mm}$ fan produced even better results.

The system is dependent on the speed at which the sensor can detect the $\mathrm{CO}_{2}$ levels. The conclusion can then be made that adding a fan is beneficial to the carbon dioxide detection system of the standoff infant apnea monitor.

\section{Multiple Fan Conclusions}

The next set of fan tests that were done were using the fans in combination to further increase the response time of the $\mathrm{CO}_{2}$ sensor. The hypothesis was that both fans would further shorten the response time of the $\mathrm{CO}_{2}$ sensor. The standoff infant apnea monitor relies on how quickly the air can get from the infant's nose to the sensor. 
Both fans were tested pushing and pulling air over and off of the sensor. Refer back to the section titled "Fan Testing" for a detailed description of the fan orientation during testing. The single $80 \mathrm{~mm}$ fan had a mean response time of 10.574 seconds, while the combined fans averaged 11.071 seconds. The p-value of 0.161 indicted that although the $80 \mathrm{~mm}$ fan was slightly faster, the results were not statistically different.

While the statistical analysis concluded no difference, the mean response time results were opposite from what was hypothesized. Both fans did not shorten the response time of the sensor. There are a few reasons why this may be. First, infants and adults have a relatively fixed lung volume, meaning they only exhale a certain amount of air. That exhaled air can be pulled over the sensor faster up to a point, but it cannot pull more air than is available. Second, the sensor works by diffusion and saturation. It is important to get the air to the sensor as fast as possible, but if it's pushed over the sensor too fast, none of the air will have a chance to diffuse into and saturate around the sensor to be detected. Infants have a smaller lung volume so this effect would be amplified when monitoring an infant. This will ultimately cause the monitor to miss breaths and not function as best it can.

From these results and conclusions the single $80 \mathrm{~mm}$ fan was chosen to pull the air from the infant's nose over the sensor. Refer back to Figure 16 to see the final system with the fan in place.

\section{Enclosure Conclusions}

The delrin enclosure serves two purposed. The first is a way to house the hardware that makes up the $\mathrm{CO}_{2}$ detection system. Instead of having to place the $\mathrm{CO}_{2}$ sensor, the fan, and the wires in different places, the enclosure allows for one piece of equipment to be mounted anywhere during testing. The enclosure can also serve to further reduce the response time. 
The results from the enclosure testing showed that the enclosure gave the $\mathrm{CO}_{2}$ sensor a slightly shorter response time. A p-value or 0.097 indicated that the results were not statistically significant. While the difference was not statistically shorter, it is also important to note that it was not statistically longer. This means that if it is used solely as a way to house the hardware the results of the $\mathrm{CO}_{2}$ detection system will not be compromised. With this in mind, the enclosure was used to house the sensor and the $80 \mathrm{~mm}$ fan.

\section{NICU Testing Conclusions}

This section will discuss the testing that was done in the NICU at Cottage Hospital in Goleta. Testing was done on one infant who had a history of infant apnea but did not have an apnea event during testing. The hypothesis being tested was that the standoff infant apnea monitor we had designed could detect infant breath from 6 inches, one foot, and two feet from the infant.

An ambient $\mathrm{CO}_{2}$ concentration was recorded in the NICU to use as a baseline to compare with the infant results. The average $\mathrm{CO}_{2}$ concentration in the NICU was $352.25 \mathrm{ppm}$. When monitoring the infant from 6 inches, one foot, and two feet, the $\mathrm{CO}_{2}$ concentrations in Table 12 were statistically higher than the baseline, as indicated by a p-value less than 0.05 . That increase is $\mathrm{CO}_{2}$ comes from the exhaled air from the infant. It can then be concluded that the monitor successfully detected the infant breath from those three tested distances. Testing was only done at those three distances because the audio system could no longer detect breath sounds past one foot.

Knowing that the infant we tested did not have any apnea events during testing, we could conclude that any events detected were false alarms. Table 12 shows the number of false alarms detected at each distance. The only false alarm that was detected was at a distance of one foot 
from the infant. There are few reasons that can be hypothesized as to why the false alarm was detected. Infants are only exhaling $15 \mathrm{ml}$ of air with each breath. If $5 \%$ of exhaled air is $\mathrm{CO}_{2}$, it means that only $0.75 \mathrm{ml}$ of the exhaled air is $\mathrm{CO}_{2}$. This is very small volume of air to detect or not detect. The sensor also has directionality, meaning it detects better if the subject is facing the sensor. If the infant turned over for even a second that small volume of $\mathrm{CO}_{2}$ would be exhaled and not detected by the sensor.

The audio system was also able to detect infant breath in the NICU setting. Audio is different from $\mathrm{CO}_{2}$ in that there are different sounds and frequencies at which those sounds are heard. $\mathrm{CO}_{2}$ is either present or absent. The frequency analysis that was done on the breath that was recorded in the NICU was particularly interesting. Dan found that infant breath had a frequency of around $15 \mathrm{kHz}$. He also could look at the breaths detected and compare them to the number of breaths an infant takes per minute. He found that the audio system was only detecting about $50 \%$ of the breaths.

The infant testing was crucial to this thesis. We had concluded that the monitor could detect adult breath, but adults exhale larger volumes of air, so it is easier to detect. The infant testing allowed us to conclude that the $\mathrm{CO}_{2}$ detection system could accurately detect infant breath from two feet away. We were only able to conclude that the whole standoff infant apnea monitor could accurately detect infant breath from 6 inches. These conclusions are based on a single set of results and further testing may lead to other conclusions.

\section{Overnight Testing Conclusions}

This section will revisit the results from the overnight testing and statistical analysis. Both the $\mathrm{CO}_{2}$ detection system alone testing and the whole system testing will be discussed. The first round of testing was done with the $\mathrm{CO}_{2}$ detection system alone. The second round of testing 
was done with both detection systems. The results can be found in Table 16 through Table 18 . Keep in mind that this round of testing was also done on an adult known to not have any form of sleep apnea. The apnea events that the system recorded were considered false alarms.

Data was collected from 15 overnight tests using the $\mathrm{CO}_{2}$ detection system alone. The number of false alarms were recorded and normalized per hour. The $\mathrm{CO}_{2}$ sensor was placed in three different orientations as described in Table 15. The orientations were compared to each other to determine which orientation detected the fewest false alarms. Orientation $\mathrm{B}_{2}$ detected the fewest false alarms per hour at 1.66. Orientation A detected the most at 3.18 per hour.

Orientation $A$ was statistically different from $B_{1}$ and $B_{2}$, but $B_{1}$ and $B_{2}$ were not statistically different from each other. From this we can conclude that placing the monitor directly above the subject will detect fewer false alarms than placing it to the left on a window sill. Both one and two feet above the test subject could detect the breath while the subject slept.

Overnight testing with the whole standoff apnea detection system was conducted over 11 nights. The $\mathrm{CO}_{2}$ system was placed in orientation $\mathrm{B}_{2}$ and the direction microphone was placed on the window sill so they would not interfere. The results showed that the whole standoff apnea monitor can statistically reduce the number of false alarms over the $\mathrm{CO}_{2}$ detection system alone. The audio detection system and the whole system produced statistically similar results seen in Table 17 and Plot 9. With the whole standoff apnea monitor an average of 0.88 apnea events per hour.

There are few speculations that can be made about the number of false alarms detected. It is rare for a person to stay one position throughout the entire night. If the subject rolled over and was breathing away from the sensor, it would have a harder time detecting the breath and result in more false alarms. During the NICU testing, the system was run for five minutes at a time, so 
false alarms would not be expected. The overnight testing was run for an average of 6.5 hours a night, increasing the chance of false alarms.

\section{Overall Conclusions}

This section will summarize the overall conclusions from all the sensor, NICU, and overnight testing. Testing was conducted on both infants and adults and valuable data was gained from both. Both audio and $\mathrm{CO}_{2}$ are good indications of breath. The standoff infant apnea monitor that was designed, using the $\mathrm{CO}_{2}$ and audio detection systems, was able to detect both adult and infant breath.

The adult testing showed that a fan can decrease the response time of the sensor. It also showed that with a simulated apnea event, the system was able to detect the event and emit the appropriate response, which was illuminating a red icon in the LabView user interface. The standoff infant apnea monitor can a simulated apnea event and alert the caregivers within the 20 seconds that is defined as an apnea event.

Infant testing showed that an increase in $\mathrm{CO}_{2}$ concentration was detected with an infant present. This meant that the infant breath was being detected by the $\mathrm{CO}_{2}$ detection system. It also showed that the combined system could accurately detect breath from 6 inches. Only the $\mathrm{CO}_{2}$ could accurately detect breath at one and two feet.

While the statistical analysis is important to be able to relate results to one another, for this thesis they were not the driving force behind design decisions. Infant apnea detection relies on how quickly the monitor can detect the lack of breath and alert the caregivers. This means every second or half second counts even if it's not statistically significant. Currently the device can detect from a distance of six inches with $100 \%$ success and one foot with $75 \%$ success. 


\section{Standoff Infant Apnea Monitor Discussions}

The purpose of this section is to discuss the selection of standoff technologies, discuss the two chosen technologies in detail, and discuss the software that was developed. This section will be very useful to students working on future generations of this monitor.

\section{Standoff Technology Selection Discussion}

This thesis focused on detecting infant apnea from a standoff approach. This meant that the monitor could not be touching the infant while it was monitoring them. The ideal system would be able to detect an infant apnea event from across the room. To get to a device that can detect breath with such precision, we had to start much closer to the infant. Our target detection distance was one to two feet away from the infant. With the information gained from background research and Dr. Barkley, we chose audio and $\mathrm{CO}_{2}$ to accurately detect the breath of an infant

from these distances. Both systems monitor the infant breath in very different ways and together they decide if an apnea event is occurring.

An infant apnea event is defined as "an unexplained episode of cessation of breathing lasting at least 20 seconds." It is important to keep in mind that infants take between 25 and 50 breaths per minute. That is a breath every 1.5 seconds. In the timeframe defined for infant apnea, 20 seconds, an infant will take on average 13 breaths. All this to say, not every breath has to be heard or measured to accurately detect an infant apnea event. If one breath is missed and the next breath is "caught" the system can still detect the breath and look for a relative change compared to the last few seconds. This is specifically important to keep in mind for monitoring the breath with the audio system. 
For the purpose of this project, two standoff technologies were sufficient. A third system could be used to initially detect the infant in the crib, such as thermal imaging. This could then start the system monitoring the infant and parents would not need to start the system manually.

\section{Audio Discussion}

The discussion about the audio system will be brief because the focus of this thesis was the $\mathrm{CO}_{2}$ infant apnea detection system. The audio is an integral part of the complete standoff infant apnea monitor, so it is important to discuss it briefly. Refer to the thesis of Dan White for a more in depth discussion about the audio system. The advantages and disadvantages to using sound as a standoff technology to monitor infant apnea will be addressed.

Audio was chosen as the primary mode of detection for the standoff infant apnea monitor. Audio allowed us to focus on sound of the air leaving the infant's nose. We chose to monitor this sound using two microphones. The first, a directional microphone, focused directly on the sound of the infant, while picking up some background noise. The second, the omnidirectional microphone, took in all the sound from the surrounding environment. The sound heard in the omni-directional microphone was then subtracted from the directional microphone in LabView, so that the infant breath was what was being used for further analysis.

Audio analysis can be used for a very large spectrum of applications; from speakers at a concert to detecting sounds not audible to the human ear. The range of frequencies that we as humans can hear stretches from 64 to $23,000 \mathrm{~Hz}$ [28]. At $15 \mathrm{kHz}$, infant breath is near the top of this range. Not all breath is heard at the same frequency making detection more difficult. An infant apnea monitor that can adapt to any breath is needed to ensure the best results. 


\section{$\mathrm{CO}_{2}$ Discussion}

$\mathrm{CO}_{2}$ was chosen as the secondary mode of detection for the standoff infant apnea monitor. It was decided that two technologies can better detect an apnea event than one technology. The follow discussion will cover the advantages and disadvantages of $\mathrm{CO}_{2}$ as a standoff technology.

For this project we focused on the air leaving the infant's nostrils. The air that is exhaled is saturated with $\mathrm{CO}_{2}$. Refer back to Figure 2 for air composition during inhalation and exhalation. Increased $\mathrm{CO}_{2}$ concentrations around the infant are a direct result of breathing, so measuring the $\mathrm{CO}_{2}$ concentration would be a good indicator of breath or lack of breath.

Monitoring infant apnea using $\mathrm{CO}_{2}$ has a few advantages. $\mathrm{CO}_{2}$ monitoring has long been used and understood in monitoring office buildings, warehouses, and the outside atmosphere. This allowed existing technology to be used, by making modifications for our application. $\mathrm{CO}_{2}$ sensors can be relatively cheap, costing between $\$ 100$ and $\$ 1000$. For the purposes of this thesis a $\$ 250$ sensor was sufficient.

$\mathrm{A} \mathrm{CO}_{2}$ detection system does have a few disadvantages to traditional apnea monitoring discussed in the section titled "Infant Apnea Hospital Monitoring." $\mathrm{CO}_{2}$ sensors rely on diffusion of the gas through the sensor to the detector. Diffusion is a slow process that depends on the concentration and force at which the substance is expelled. Infants do not have a large lung volume and they do not exhale with great force, so we are relying on a small volume of air to diffuse across the sensor within the timeframe of 20 seconds. Modifications need to be made to increase the rate of diffusion of the exhaled breath. For this thesis, an $80 \mathrm{~mm}$ USB fan was used to help move the air over the sensor. Through testing, this was deemed to be a success in shortening the time from the exhalation to the $\mathrm{CO}_{2}$ sensor detecting the breath. 


\section{LabView Software Discussion}

This thesis included both hardware and software design to monitor infant apnea using standoff technologies. The software was designed using National Instruments LabView. LabView is a visual software developer that uses "blocks" to write the code instead of traditional programming language. A detailed description of LabView was given in the "Monitor Design" section. The Vernier $\mathrm{CO}_{2}$ sensor that was purchased for this thesis easily integrated with LabView using the Vernier SensorDAQ that was also purchased.

The audio and $\mathrm{CO}_{2}$ detection systems software were designed separately using LabView. Once complete, they were integrated by copying one code and pasting it in the block diagram of the other code. The two could still be run independently, if desired, but they could also be run as a complete standoff infant apnea monitor. A small portion of LabView code was added to allow the two detection systems to communicate on whether each was detecting apnea, and then decide on whether a true apnea event was occurring.

Once the software was finalized for the proof-of-concept standoff infant apnea monitor, it was turned into an executable file (.exe) that could be run on any computer. This eliminated the need for LabView to be uploaded onto the Netbooks that were used. LabView takes up a considerable amount of hard drive space and the Netbooks did not have the capacity to run LabView. The executable file allowed the user interface to be used without access to the block diagram. This ensured no code would be changed while the program was running. Ideally, this executable file could be run on a small device such as a PDA or iPhone.

LabView also allowed us to design a very user-friendly interface. The interface included graphs of both detection systems in real-time, logs recording apnea events, and a color changing 
icon based on if an apnea event was detected or not. Refer back to Figures 22, 23, and 28 for an image of the user interface.

\section{Integrated System Discussion}

The $\mathrm{CO}_{2}$ LabView software was combined with the audio LabView software to create one system that would monitor infant breath and determine if the infant was suffering an apnea event with time to alert the parents. This was done with ease using LabView. The block diagrams did not have to be reconstructed in a separate file. The $\mathrm{CO}_{2}$ block diagram was highlighted and "dragged" into the open space on the block diagram of the audio system. A small section of additional code was added to the final system to utilize both detection systems. If the $\mathrm{CO}_{2}$ and the audio system do not detect breath, the overall detection system light would turn read and read "APNEA." The apnea event would also be recorded in the "Logged Events" tab for future viewing. If only one system detect an apnea event the light would remain green to indicate breathing.

Our data showed that two systems working together can have fewer false alarms that one system working alone. It is predicted that the same would hold true for missing fewer true apnea events using two systems instead of one. Adding a third system may further enhance the abilities of the standoff infant apnea monitor, but further testing would need to be done to make this conclusion. 


\section{Difficulties}

This section will discuss the difficulties that were faced during the duration of this thesis. No major setbacks were faced during the length of this project. Both Cal Poly and Raytheon worked well together to minimize setbacks. The two areas where there were some difficulties were availability of infants to test and allotted funds for the project.

\section{Infant Testing}

The objective of this thesis was to design a monitor that could detect infant apnea events without touching the infant. This scope required testing to be done on infants. This aspect of the project was difficult because our main connection to infants was down in Goleta, about 1.5 hours away from us. About midway through the project, I placed a call locally to Dr. VanScoy at Sierra Vista Regional Medical Center. He was willing to aid us with the testing of the device but because he did not work directly for the hospital, he could not give the ok. We had to go through the hospital review board to get approval to test on the infants in the NICU at Sierra Vista Regional Medical Center. After much discussion, we decided that this was not an efficient use of time with the June deadline we had. We decided to continue doing testing on adult subjects and travel to Goleta when we could. Dr. Barkley is the head of the NICU at Cottage Hospital, so it was easier for him to make the call to allow us to test there.

Dr. Barkley was very helpful in allowing us to test our device on the infant in his NICU. We were able to record one infant during our second trip to Goleta. The Cottage Hospital NICU did not have any infants that they were monitoring for infant apnea, during the time of our testing. This meant we could only use the infants to ensure that our device could detect their 
breath. This was discussed with Raytheon and Dr. Laiho and determined to be sufficient in combination with the adult testing that was done.

\section{Adult Testing}

None of the adults that were used for testing had any form of sleep apnea. This limited our testing to false alarm detection. If the standoff infant apnea monitor detected an apnea event during a round of testing it was concluded to be a false alarm. The advantage of testing adults without apnea was that if there was a detected apnea event we did not have to wonder if it was a true apnea event or a false alarm.

The initial adult testing was conducted in such a way that the test subject only breathed out their nose to make the testing better parallel infant testing. The adult was awake and could control breathing out of their nose. When the overnight testing was done the adult subject was asleep. There was no way to ensure they only breathed out of their nose. The overnight results would only be applicable to an adult sleep apnea scenario. To obtain overnight results applicable to infant apnea, testing would need to be preformed solely on infants.

\section{Software}

National Instruments LabView made the software design and integration of the two systems virtually pain free. The main difficulty is that different versions of LabView, such as 8.0 or 8.2 , are not directly compatible with each other. If the software is designed in version 8.2 by one person, and is designed in another version by another, there are a number of steps that have to be taken to get the programs to run together. For future work on the infant apnea detection software it would be advised to construct everything in one version to avoid the problems of compatibility. 


\section{Budget Restrictions}

One of our biggest constraints was our budget. We were allotted \$3,500. Many of the researched standoff technologies required hardware that was well above this budget. The project was a proof-of-concept device, so it was important that we purchase a lot of different hardware to ensure that our final design was the best it could be. Both of the standoff technologies chosen, audio and $\mathrm{CO}_{2}$, required hardware that was within our budget. We also wanted to keep in mind that if this device did go to market it needed to be a reasonable price for parents to want to purchase it. 


\section{Future Work and Next Steps}

This section will suggest future directions for the success of the standoff infant apnea monitor. Continued testing will be discussed first. Next discussed will be suggested hardware and software modifications. The standoff infant apnea monitor has potential to be a marketable product. This section will also discuss the other avenues that this research may be useful. Raytheon has agreed to sponsor another round of funding for this project, so this section may be useful to future students.

\section{Continued Infant Testing}

With this thesis being a proof-of-concept prototype, there is a lot of room for improvement and refining in future phases. The immediate testing needs would be 1) overnight testing of healthy infants, 2) continuous testing on infants known to have apnea, 3) more overnight testing on adults with apnea.

We obtained very useful data from the healthy infant at Cottage Hospital that helped us conclude that we could use the audio and carbon dioxide systems to monitor infant breath. Future testing on healthy infants would allow us to refine the hardware and software to obtain greater standoff distances. By testing healthy infants, any apnea event recorded is a false alarm, so the focus can be on decreasing false alarms and increasing accuracy of the device before testing on infants with apnea.

This device was designed to detect infant apnea, so the greatest knowledge would come from testing infants with known apnea conditions. This was not in the scope of this thesis, but could also be done as a study at Cottage Hospital with hospital board approval. The infants would be continually monitored by the current proven technology, while being monitored with 
our standoff infant apnea monitor. The data collected can be compared to the proven systems to look for detected apnea events, false apnea events, or missed events. With previous testing at Cottage Hospital, we showed that our device does not interfere with any current technologies in the NICU, so the complexity of the study would be very low. There is nothing invasive about the testing, making hospital consent much easier.

The intermediate step between infant testing and adult testing would be testing of young children. They have lung volumes and respiratory features closer to an infant, so the result could be easier extrapolated to infant applications. This data could be easily collected from healthy children in a home setting while they are asleep.

Another avenue of continued testing would be adult testing. If the device can monitor infant breath, then it can monitor adult breath, because adult breath is much easier to detect, due to larger volumes or air. Adult testing would be more accessible and could be done in a home setting. This testing would greatly benefit from a video camera running alongside the apnea monitor. The results can be compared to different orientations of the subject while sleeping and detected apnea events can be seen on a video to better understand if a true apnea event was occurring or if a false alarm was detected.

\section{$\mathrm{CO}_{2}$ Hardware Improvements}

This section will discuss the next steps that can be implemented with the $\mathrm{CO}_{2}$ detection system hardware. The data collected from this thesis can help in the next generation $\mathrm{CO}_{2}$ hardware of the standoff infant apnea monitor. New systems can be purchased with specific specifications in mind.

Purchasing new sensing equipment would be the first step to improving the $\mathrm{CO}_{2}$ apnea detection system. The sensor that was purchased is used in a classroom setting with kids using it 
and dropping it, so it was made more robust than for research purposes. The sensor needs to read $\mathrm{CO}_{2}$ concentrations in real time, meaning every second, if not faster. The more samples the sensor could read per second would enhance the accuracy of the overall apnea detection system. Future modifications could be made to the existing senor. By researching exactly how the sensor works and what components are most critical, it may be stripped of unnecessary hardware to increase the sensitivity and miniaturize the system.

Currently the alert for the parents is built into the LabView software and is shown on the user interface. Ideally this would either be a small receiver that can fit in a pocket, so the parent can take it around the house. The apnea monitor on the side of the crib would send a signal to the receiver, in the form of a sound or vibration to let them know their child is in distress. Once a wireless receiver had been achieved, the next step would be syncing the monitor with a computer or phone, so no extra components are necessary to carry around. This could be in the form of an application for a phone or computer.

Once the new hardware is purchased, a miniaturized system that fits on the side a crib would be ideal. The crib side module would need to have nothing that could injure the infant if they pulled it off. It should have a small screen so the caregivers can view different setting. It should also have a port on the side that data can be downloaded from the module to a computer for future analysis by a physician. These features may not be directly in the next generation device, but would be beneficial before the device goes to market.

\section{$\mathrm{CO}_{2}$ Software Improvements}

Ideally the new hardware would integrate with LabView, so as to build off the existing software platform. The way the baseline is established could be improved. If the system can constantly be taking an average of the last ten seconds and comparing each new value to the last 
ten seconds, false alarms may be able to be further reduced, while decreasing missed apnea events. Another improvement would be writing the logged apnea events to a file that could be accessed by Microsoft Word or Excel for future analysis. The current system allows the logged events to be viewed, but they have to be manually transferred for further analysis. If the $\mathrm{CO}_{2}$ system could increase the awareness of the audio system, less apnea events may also be missed.

If no breath is detected by the $\mathrm{CO}_{2}$ system, it would be ideal if it triggered the audio system to be looking more closely at the sound for any sign of apnea.

\section{Other Applications of Researched Standoff Technologies}

Using standoff technologies to monitor vital signs has great potential in other areas besides infant or adult apnea. Some other avenues that this research may be useful include: monitoring patients in a hospital setting without all the leads and other necessary wires, interrogation, and remote detection of army personnel during combat situations.

Currently in hospitals, monitoring patients requires many leads and wires. This can be uncomfortable for the patient and their loved ones coming to visit. It would seem beneficial to the patients both mentally and physically to be able to recover in a more "natural" environment without having wires attached, while still being monitored.

When researching standoff technologies, interrogation came up as an application, specifically related to infrared imaging. Currently, during a lie detector test the person being interrogated has a pulse oximeter on their finger and an ECG to monitor heart rate. Heart rate, which is also tied to respiration rate, has been a proven indicator of lying. If you can use the infrared technology discussed in the "Background" section of this paper to measure respiration rate, you may only need a camera to tell if someone is lying. 
It would be very beneficial if the military could remotely monitor soldiers and other military personnel in combat situations. If a roadside bomb goes off and soldiers are wounded or killed, it is important to relay the welfare of the soldiers to rescue crews. With remote detection of vital signs, rescue personnel would be able to best assess the situation. If there are critically wounded soldiers then the rescue crews know the situation is urgent. If the soldiers are showing stable vital signs or worse case, if they are dead, the rescue crews can wait to move in and eliminate the potential threat on their lives. 


\section{Works Cited}

1. Debertin, M.S.M.D.A.S., The German case-control scene investigation study on SIDS: epidemiological approach and main results. International Journal of Legal medicine, 2009.

2. Fetus, C.o. and Newborn, Apnea, Sudden Infant Death Syndrome, and Home Monitoring. Pediatrics, 2003. 111(4): p. 914-917.

3. Marieb, E.N., ed. Anatomy and Physiology. 2nd ed. 2005, Pearson Education, Inc. : San Francisco. 735 - 746.

4. Murthy, R. and I. Pavlidis, Noncontact measurement of breathing function. Engineering in Medicine and Biology Magazine, IEEE, 2006. 25(3): p. 57-67.

5. $\quad$; Available from: http://emsresource.net/vitals.shtml.

6. Praud, J.-P. and P. Reix, Upper airways and neonatal respiration. Respiratory Physiology \& Neurobiology, 2005. 149(1-3): p. 131-141.

7. Tidal Volume, in Encyclopaedia Britannica. 2002.

8. White, D.P., Pathogenesis of Obstructive and Central Sleep Apnea. Am. J. Respir. Crit. Care Med., 2005. 172(11): p. 1363-1370.

9. Frappell, P.B. and P.M. MacFarlane, Development of mechanics and pulmonary reflexes. Respiratory Physiology \& Neurobiology, 2005. 149(1-3): p. 143-154.

10. Shiroh Isono, A.T., Teruhiko Ishikawa, and Takashi Nishino, Developmental Changes in Collapsibility of the Passive Pharynx during Infancy. American Journal of Respiratory Critical Care Medicine, 2000. 162: p. 832-836.

11. Young, T., P.E. Peppard, and D.J. Gottlieb, Epidemiology of Obstructive Sleep Apnea: A Population Health Perspective. Am. J. Respir. Crit. Care Med., 2002. 165(9): p. 12171239. 
12. Erler, T. and A. Peters, Electronic home monitoring of infants-A recurring topic. Somnologie - Schlafforschung und Schlafmedizin, 2006. 10(1): p. 3-9.

13. AngelCare Infant Apnea Monitor. May 10, 2009]; Available from: http://www.btbabymonitors.com/angelcare.

14. Respisense BUZZ Infant Breathing Movement Baby Monitor. May 10, 2009]; Available from: http://www.respisense.com/en/index.php.

15. PhD., D.S.B., Discussion on Infant Apnea. 2008: Cottage Hospital Goleta, CA.

16. LEUNG, R.S.T. and T. DOUGLAS BRADLEY, Sleep Apnea and Cardiovascular Disease. Am. J. Respir. Crit. Care Med., 2001. 164(12): p. 2147-2165.

17. Willinger M, J.L., Catz C, Defining the sudden infant death syndrome (SIDS): Deliberations of an expert panel convened by the National Institute of Child Health and Human Development. Pediatric Pathology, 1991. 11: p. 677-684.

18. Coleman-Phox, K., R. Odouli, and D.-K. Li, Use of a Fan During Sleep and the Risk of Sudden Infant Death Syndrome. Arch Pediatr Adolesc Med, 2008. 162(10): p. 963-968.

19. Zhen, Z., F. Jin, and I. Pavlidis. Tracking human breath in infrared imaging. in Bioinformatics and Bioengineering, 2005. BIBE 2005. Fifth IEEE Symposium on. 2005.

20. Greneker, E.F. Radar sensing of heartbeat and respiration at a distance with applications of the technology. in Radar 97 (Conf. Publ. No. 449). 1997.

21. PASTERKAMP, H., STEVE S. KRAMAN, and GEORGE R. WODICKA, Respiratory Sounds . Advances Beyond the Stethoscope. Am. J. Respir. Crit. Care Med., 1997. 156(3): p. $974-987$.

22. Taplidou, S.A. and L.J. Hadjileontiadis, Wheeze detection based on time-frequency analysis of breath sounds. Computers in Biology and Medicine, 2007. 37(8): p. 10731083.

23. Tans, D.P., Trends in Atmospheric Carbon Dioxide, U.S.D.o. Commerce, Editor. 2010, NOAA. 
24. SenseAir, Carbon Dioxide - Life and Death. 2000.

25. Tobias, J.D. and D.J. Meyer, Noninvasive monitoring of carbon dioxide during respiratory failure in toddlers and infants: end-tidal versus transcutaneous carbon dioxide. Anesthesia \& Analgesia, 1997. 85(1): p. 55-58.

26. Thys, F., et al., PaCO2/ETCO2 gradient: early indicator of thrombolysis efficacy in a massive pulmonary embolism. Resuscitation, 2001. 49(1): p. 105-108.

27. Technology, V.S.a., $\mathrm{CO}_{2}$ Gas Sensor. 2007: Beaverton, OR.

28. Popper, R.F.A., ed. Comparative Hearing: Mammals Springer Handbook of Auditory Research Series 1994: Springer-Verlag, NY. 